\title{
ON REPRESENTATIONS OF RATIONAL CHEREDNIK ALGEBRAS OF COMPLEX RANK
}

\author{
INNA ENTOVA AIZENBUD
}

\begin{abstract}
We study a family of abelian categories $\underline{\mathcal{O}}_{c, \nu}$ depending on complex parameters $c, \nu$ which are interpolations of the category $\mathcal{O}$ for the rational Cherednik algebra $H_{c}(\nu)$ of type $A$, where $\nu$ is a positive integer. We define the notion of a Verma object in such a category (a natural analogue of the notion of Verma module).

We give some necessary conditions and some sufficient conditions for the existence of a non-trivial morphism between two such Verma objects. We also compute the character of the irreducible quotient of a Verma object for sufficiently generic values of parameters $c, \nu$, and prove that a Verma object of infinite length exists in $\mathcal{O}{ }_{c, \nu}$ only if $c \in \mathbb{Q}<0$. We also show that for every $c \in \mathbb{Q}<0$ there exists $\nu \in \mathbb{Q}<0$ such that there exists a Verma object of infinite length in $\mathcal{O}_{c, \nu}$.

The latter result is an example of a degeneration phenomenon which can occur in rational values of $\nu$, as was conjectured by P. Etingof.
\end{abstract}

\section{INTRODUCTION}

The study of representations in complex rank involves defining and studying families of abelian categories depending on a parameter $t$ which are polynomial interpolations of the categories of representations of objects such as finite groups, Lie groups, Lie algebras and more. This was done by P. Deligne in [6] for finitedimensional representations of the general linear group $G L_{n}$, the orthogonal and symplectic groups $O_{n}, S p_{2 n}$ and the symmetric group $S_{n}$. Deligne defined Karoubian tensor categories $\operatorname{Rep}\left(G L_{t}\right), \operatorname{Rep}\left(O S p_{t}\right), \operatorname{Rep}\left(S_{t}\right), t \in \mathbb{C}$, which in points $n=t \in \mathbb{Z}_{+}$ admit an essentially surjective functor onto the standard categories $\operatorname{Rep}\left(G L_{n}\right)$, $\operatorname{Rep}\left(O S p_{n}\right), \operatorname{Rep}\left(S_{n}\right)$. The category $\operatorname{Rep}\left(S_{t}\right)$ was subsequently studied by himself and others (F. Knop in [16, V. Ostrik, J. Comes in [5]).

The resulting categories can be shown to have superexponential growth, and are not equivalent to the category of finite-dimensional representations of any affine algebraic group or supergroup. One can look for rational non-integer values of $t$ where degeneration occurs, which would allow us to understand better how the structure of the classical category of representations is similar for all ranks $n$, and why degenerate phenomena occur at specific values of $n$.

Received by the editors March 17, 2014 and, in revised form, June 14, 2014 and September 17, 2014 .

2010 Mathematics Subject Classification. Primary 16S99 and 18D10.

Key words and phrases. Deligne categories, rational Cherednik algebra. 
P. Etingof, in [9], suggested using these results to define and study the categories of representations of rational Cherednik algebras, as well as Lie superalgebras, affine Lie algebras and other "non-compact" representation theory objects. A possible approach, as stated in [9], would be using the tensor categories $\operatorname{Rep}\left(G L_{t}\right), \operatorname{Rep}\left(O S p_{t}\right), \operatorname{Rep}\left(S_{t}\right)$ and defining the required category for a "non-

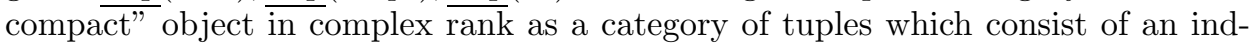
object of the corresponding tensor category along with morphisms satisfying some relations.

In this paper, we study the categories which are interpolations of the classical $\mathcal{O}$-category of lowest weight modules for the rational Cherednik algebra of type $A$, denoted by $H_{c}(n)$. These categories are BGG-type categories, where one can define Verma objects and study morphisms between these, as well as their irreducible quotients.

The categories $\operatorname{Rep}\left(H_{c}(\nu)\right)$ (the parameter $\nu$ replacing the parameter $t$ used above) have been defined in [9] as categories whose objects are ind-objects of Deligne's category $\operatorname{Rep}\left(S_{\nu}\right)$ with additional structure; namely, two morphisms in $\underline{\operatorname{Rep}}\left(S_{\nu}\right)$, denoted by $x, y$, satisfying some relations. These morphisms represent the actions of the elements $x_{1}, \ldots, x_{n}$ and $y_{1}, \ldots, y_{n}$ of $H_{c}(n)$ respectively. We proceed to define the full subcategory $\underline{\mathcal{O}}_{c, \nu}$ of $\operatorname{Rep}\left(H_{c}(\nu)\right)$, which is an interpolation of the category $\mathcal{O}$ of the classical Cherednik algebra $H_{c}(n)$.

In this category $\underline{\mathcal{O}}_{c, \nu}$ we have objects we will call "Verma objects", as they are analogues of the Verma modules in the classical category $\mathcal{O}$. They are parameterized by arbitrary partitions $\lambda$, and denoted by $M_{c, \nu}(\lambda)$. As in the $H_{c}(n)$ case, they have a natural grading as $\operatorname{Rep}\left(S_{\nu}\right)$ ind-objects, with the lowest grade consisting of an

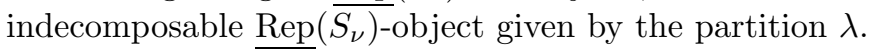

The Verma objects have about the same properties as the classical Verma modules, and one can ask for which values of parameters $c, \nu$ they are reducible, and for which values of parameters $c, \nu$ there are non-trivial morphisms between different Verma objects. It would also be interesting to compute the character of the unique irreducible quotient of $M(\lambda)$ (the character being its decomposition as a $\underline{\operatorname{Rep}}\left(S_{\nu}\right)$ ind-object into a sum of irreducible $\underline{\operatorname{Rep}}\left(S_{\nu}\right)$-objects).

1.1. Summary of the results. Recall that the category $\operatorname{Rep}\left(S_{\nu}\right)$ is a Karoubian rigid symmetric monoidal category which is abelian semisimple whenever $\nu \notin \mathbb{Z}_{+}$; for any $\nu$, its indecomposable objects are parameterized by Young diagrams of arbitrary size. One can treat an indecomposable object $X_{\tau}$ of $\operatorname{Rep}\left(S_{\nu}\right)$, with $\tau$ being a Young diagram, as corresponding to the "virtual" Young diagram of size $\nu$ obtained from $\tau$ by adding a very long top row ("of size $\nu-|\tau|$ ").

Throughout the paper we will assume that $\nu \notin \mathbb{Z}_{+}$unless it is explicitly stated otherwise.

The indecomposable object in $\operatorname{Rep}\left(S_{\nu}\right)$ corresponding to a Young diagram with one box is denoted by $\mathfrak{h}_{0}$, and has dimension $\nu-1$ (it corresponds to the reflection representation of $S_{n}$ when $\left.\nu=n\right)$. One can define an algebra object $S \mathfrak{h}_{0}^{*}$ in ind $\underline{\operatorname{Rep}}\left(S_{\nu}\right)$ (which would correspond to the ring $\mathbb{C}\left[x_{1}, \ldots, x_{n}\right] /\left\langle\sum_{i} x_{i}\right\rangle$ when $\nu=n$ ).

We define the Verma objects $M_{c, \nu}(\lambda)$ of $\underline{\mathcal{O}}_{c, \nu}$ in Subsection 6.2 (these are sometimes denoted $M(\lambda)$ for short). The underlying ind-object of $\operatorname{Rep}\left(S_{\nu}\right)$ is just $S \mathfrak{h}_{0}^{*} \otimes X_{\lambda}$. 
For every non-negative integer $n$, we construct a functor $\mathcal{F}_{c, n}$ from $\operatorname{Rep}\left(H_{c}(\nu=\right.$

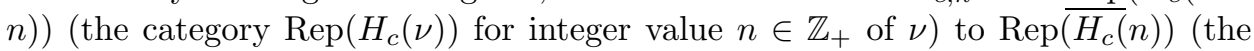
category of representations of $\left.H_{c}(n)\right)$. Considering the full subcategory of $\underline{\mathcal{O}}_{c, \nu=n}$ whose objects are $M_{c, \nu=n}(\lambda)$ such that $|\lambda| \leq n / 2$, we see that the restriction of $\mathcal{F}_{c, n}$ to this subcategory is fully faithful; in fact, $\mathcal{F}_{c, n}$ takes Verma object $M_{c, \nu=n}(\lambda)$ to Verma module in $\mathcal{O}\left(H_{c}(n)\right)$ whose lowest weight corresponds to the Young diagram obtained from $\lambda$ by adding a top row of size $n-|\lambda|$.

We would like to understand the structure of the Verma objects $M_{c, \nu}(\lambda)$ of the category $\underline{\mathcal{O}}_{c, \nu}$. We start by introducing the following notation:

Notation 1.1.1.

- Denote the set of points $(c, \nu)$ on the complex plane where $M_{c, \nu}(\tau)$ is reducible by $\mathrm{B}_{\tau}$. The image of $\mathrm{B}_{\tau} \backslash\{c=0\}$ under the map $c \mapsto 1 / c$ will be denoted by $\mathcal{B}_{\tau}$.

- Denote the set of points $(c, \nu)$ on the complex plane where there is a nonzero morphism $M_{c, \nu}(\mu) \longrightarrow M_{c, \nu}(\tau)$ by $\mathrm{B}_{\mu, \tau}$. The image of $\mathrm{B}_{\mu, \tau} \backslash\{c=0\}$ under the map $c \mapsto 1 / c$ will be denoted by $\mathcal{B}_{\mu, \tau}$.

The first step in studying the reducibility of the Verma objects $M_{c, \nu}(\lambda)$ would be describing the sets $\mathrm{B}_{\tau}$ (resp. $\mathcal{B}_{\tau}$ ).

As in the classical case, we have: $\mathrm{B}_{\tau}=\bigcup_{\mu} \mathrm{B}_{\mu, \tau}$, the parameter $\mu$ running over all Young diagrams of arbitrary size. This allows us to concentrate on studying the sets $\mathrm{B}_{\mu, \tau}\left(\operatorname{resp} . \mathcal{B}_{\mu, \tau}\right)$.

We will show that if $\mu \neq \tau$, the set $\mathrm{B}_{\mu, \tau}$ lies inside a countable (disjoint) union of curves $\bigcup_{m \in \mathbb{Z}_{>0}} \mathrm{~L}_{\tau, \mu, m}$ for which we give explicit equations (cf. Section 8 ). These curves become straight lines, denoted by $\mathcal{L}_{\tau, \mu, m}$, when we switch to parameters $(1 / c, \nu)$ instead of $(c, \nu)$.

For a fixed positive integer $m$, the following condition on a point $(c, \nu)$ implies $(c, \nu) \in \mathrm{L}_{\tau, \mu, m}$ :

Condition 1.1.2. There exists a non-zero map $M_{c, \nu}(\mu) \longrightarrow M_{c, \nu}(\tau)$ with the lowest grade of $M_{c, \nu}(\mu)$ mapping to grade $m$ of $M_{c, \nu}(\tau)$.

It is easy to see that the intersection of the set $\mathcal{B}_{\mu, \tau}$ with each of these lines $\mathcal{L}_{\tau, \mu, m}$ is either the entire line or a finite number of points. As a step towards decribing the set $\mathcal{B}_{\mu, \tau}$, we give a full description of the straight lines $\mathcal{L}_{\tau, \mu, m}$ lying inside $\mathcal{B}_{\mu, \tau}$ whenever $|\tau| \neq|\mu|$ in Theorem 1 .

Recall that in the theory of classical rational Cherednik algebras, the existence of a non-zero map between Verma modules $M_{c, n}(\tau), M_{c, n}(\mu)$ depends on whether $\tau, \mu$ have the same $e$-core, where $e$ is an integer depending on $n, c$.

Therefore, in order to understand when there is a non-zero map between two given Verma objects in $\underline{\mathcal{O}}_{c, \nu}$, we would like to explain what it means for two virtual Young diagrams of size $\nu$ to have the same core.

Let $\tau$ be a Young diagram. As we explained before, we have the corresponding virtual Young diagram $\tilde{\tau}(\nu)$ obtained by adding a very long top row of size $\nu-|\tau|$ to $\tau$.

Define $C_{\tau}$ to be the set of integers $\left\{|\tau|-1+j-\check{\tau_{j}} \mid j \in \mathbb{Z}_{>0}\right\}$ (here $\check{\tau}$ is the transpose of $\tau$, and so $\check{\tau_{j}}$ is the length of the $j$-th column of $\tau$ ).

The set $C_{\tau}$ is the set of all integers $s$ for which the virtual Young diagram $\tilde{\tau}(\nu)$ has a hook of length $\nu-s$ (this is again a "virtual" hook, with a "very long arm", and a leg of length $\widetilde{\tau_{j}}$ ). 
When this virtual hook is removed, we obtain a "normal" Young diagram of size $s$, denoted by $\operatorname{core}_{(\nu-s)}(\tau)$. Inserting into $\operatorname{core}_{(\nu-s)}(\tau)$ another virtual hook of size $\nu-s$ with leg $\check{\tau_{j}}+l$ (for any integer $l \geq-\widetilde{\tau_{j}}$ ), we obtain a new virtual Young diagram of size $\nu$.

Removing the very long top row of the new virtual Young diagram, we obtain a ("normal") Young diagram which we will call $\Gamma(\tau, s, l)$.

The conditions under which there exists a non-zero map between Verma modules for $H_{c}(n)$ allow us to expect that there exists a non-zero morphism $M_{c, \nu}(\mu) \longrightarrow$ $M_{c, \nu}(\tau)$ only if $\mu=\Gamma(\tau, s, l)$ for some $l$. In fact, we prove the following:

Theorem 1. For two distinct Young diagrams $\mu, \tau$ and an integer $m>0$, the following are equivalent:

(1) $|\mu| \neq|\tau|$ and $\mathcal{L}_{\tau, \mu, m} \subset \mathcal{B}_{\mu, \tau}$,

(2) $\mu=\Gamma(\tau, s, \operatorname{sign}(|\mu|-|\tau|))$ for some $s \in C_{\tau}$ (in particular, $|\mu| \neq|\tau|$ ), and $(|\mu|-|\tau|) \mid m$.

One can easily see that if $\mu, \lambda, m$ satisfy the conditions of the above theorem, then for a generic point $\left(\frac{1}{c}, \nu\right)$ of $\mathcal{L}_{\tau, \mu, m}$, the Verma object $M_{c, \nu}(\tau)$ has length 2 .

In such a case, we give a formula for the character of the simple object $L_{c, \nu}(\tau)$; moreover, we show that there exists a long exact sequence

$$
\begin{aligned}
\cdots \rightarrow M(\Gamma(\tau, s, \pm l)) & \rightarrow M(\Gamma(\tau, s, \pm(l-1))) \rightarrow \cdots \rightarrow M(\Gamma(\tau, s, \pm 1)=\mu) \\
& \rightarrow M(\tau) \rightarrow L(\tau) \rightarrow 0 .
\end{aligned}
$$

The sign is $\operatorname{sign}(|\mu|-|\tau|)$, and this is a finite sequence if $|\mu|<|\tau|$.

The character formula for $L_{c, \nu}(\tau)$ is then obtained from Euler's formula applied to the above long exact sequence.

In particular, we compute explicitly the character of the simple object $L_{c, \nu}(\emptyset)$, whenever $(1 / c, \nu)$ is a generic point on a line $\mathcal{L}_{\emptyset, \mu, k}=\{(1 / c, \nu) \mid c \nu=k\}$ (here $k \in \mathbb{Z}_{>0}$ is fixed).

We conclude the paper by proving the following theorem:

Theorem 2. If there exists a Verma object of infinite length in $\underline{\mathcal{O}}_{c, \nu}$, then $c \in \mathbb{Q}<0$. In fact, one can show that for any $c \in \mathbb{Q}_{<0}$, there exists $\nu \in \mathbb{Q}_{<0}$ such that $M_{c, \nu}(\emptyset)$ has infinite length.

Theorem 2 is an example of a degenerate phenomenon which can occur for rational non-integer values of $\nu$. The classical representation theory of Cherednik algebras says that for a non-negative integer $n$, all modules in $\mathcal{O}\left(H_{c}(n)\right)$ have finite length (cf. [10, Corollary 3.26]), and we see from Theorem 2 that the same is true for Verma objects in $\underline{\mathcal{O}}_{c, \nu}$ for $c \notin \mathbb{Q}$.

It would be interesting to know about other phenomena which can occur in $\underline{\mathcal{O}} c, \nu$ only for $\nu \in \mathbb{Q} \backslash \mathbb{Z}_{+}$. Such phenomena were conjectured by P. Etingof in $[9]$.

1.2. Structure of the paper. In Section 3 , we recall basic definitions and facts about $H_{c}(n)$, the Cherednik algebra of type $A$. Some further facts about the blocks of the $\mathcal{O}$-category for $H_{c}(n)$ will be given in Section 9 .

In Section 4 we recall basic facts about the Deligne category $\operatorname{Rep}\left(S_{\nu}\right)$. We do not give a definition (it can be found in [6]), instead we mention the facts about this category which we will use. 
In Sections 5 and 6, we define the category $\operatorname{Rep}\left(H_{c}(\nu)\right)$ (interpolation of the category of representations of $\left.H_{c}(n)\right)$ and the category $\underline{\mathcal{O}}_{c, \nu}$ (interpolation of the $\mathcal{O}$-category of $\left.H_{c}(n)\right)$. We then define the Verma objects $M_{c, \nu}(\tau)$ in $\underline{\mathcal{O}}_{c, \nu}$.

In Section 7 we discuss the functor from $\operatorname{Rep}\left(H_{c}(\nu=n)\right)$ (the category $\operatorname{Rep}\left(H_{c}(\nu)\right)$ for integer value $n \in \mathbb{Z}_{+}$of $\nu$ ) to $\operatorname{Rep}\left(H_{c} \overline{(n))}\right.$ (the category of representations of $\left.H_{c}(n)\right)$. In Section 8, we give a necessary condition on $(c, \nu)$ which is required in order for a non-trivial map $M_{c, \nu}(\mu) \longrightarrow M_{c, \nu}(\tau)$ to exist. This condition is an equation which defines the lines $\mathcal{L}_{\tau, \mu, m}$.

In Section 10 we define constructions for $\underline{\mathcal{O}}_{c, \nu}$ which are necessary in order to describe the triplets $(\tau, \mu, m)$ for which $\mathcal{L}_{\tau, \mu, m} \subset \mathcal{B}_{\mu, \tau}$ (the latter is done in Section [11] we give a full description in the case $|\tau| \neq|\mu|$, and a partial description when $|\tau|=|\mu|)$.

In Section 12 we define the formal character of an object of $\underline{\mathcal{O}} c, \nu$, give a formula for the character of a Verma object $M_{c, \nu}(\tau)$, and compute the character of a simple object $L_{c, \nu}(\tau)$ in the simplest cases. We also give a positive character formula for the Verma objects and look at examples when such a formula can be derived for some simple objects $L_{c, \nu}(\tau)$.

In Section 13 we prove Theorem 2 mentioned above, and in particular, give a lower bound on the grade of a given Verma object $M_{c, \nu}(\tau)$ in which a simple $\operatorname{Rep}\left(S_{\nu}\right)$-object $X_{\mu}$ can lie.

\section{Notation And CONVEntions}

The base field throughout this paper will be $\mathbb{C}$.

Notation 2.0.1. For a rational number $q \in \mathbb{Q}$, we write $q=\frac{\operatorname{num}(q)}{\operatorname{den}(q)}$, where $\operatorname{num}(q)$, $\operatorname{den}(q) \in \mathbb{Z}, \operatorname{den}(q)>0, \operatorname{gcd}(\operatorname{num}(q), \operatorname{den}(q))=1$.

\subsection{Symmetric group and Young diagrams.}

Notation 2.1.1.

- $S_{n}$ will denote the symmetric group $\left(n \in \mathbb{Z}_{+}\right)$. We will denote by $S$ the set of reflections $s_{i j}$ in $S_{n}$.

- The notation $\lambda$ will stand for a partition (weakly decreasing sequence of non-negative integers), a Young diagram $\lambda$ (considered in the English notation), and the corresponding irreducible representation of $S_{|\lambda|}$. Here $|\lambda|$ is the sum of entries of the partition, or, equivalently, the number of cells in the Young diagram $\lambda$. We will sometimes write $\lambda \vdash n$ to say that $\lambda$ is a partition of $n$. We will denote the set of all Young diagrams of arbitrary size by $\mathcal{P}$.

- When referring to a cell in a Young diagram $\lambda,(i, j)$ will be the cell in row $i$ and column $j$, with $i, j \geq 1$.

- The length of the partition $\lambda$, i.e. the number of rows of Young diagram $\lambda$, will be denoted by $l(\lambda)$.

- The $i$-th entry of a partition $\lambda$, as well as the length of the $i$-th row of the corresponding Young diagram, will be denoted by $\lambda_{i}$ (if $i>l(\lambda)$, then $\lambda_{i}:=0$ ). The transpose of the Young diagram $\lambda$ will be denoted by $\lambda^{2}$ (so the length of the $i$-th column of $\lambda$ is $\lambda_{i}$ ).

Example 2.1.2. Consider the Young diagram $\lambda$ corresponding to the partition $(6,5,4,1)$. The length of $\lambda$ is 4 , the size is 16 , and the transpose of 
$\lambda$ is the Young diagram corresponding to partition $(4,3,3,3,2,1)$ :

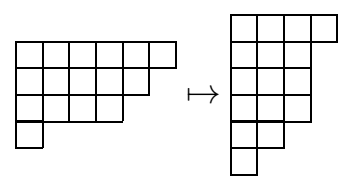

- $\mathfrak{h}$ (in context of representations of $S_{n}$ ) will denote the permutation representation of $S_{n}$, i.e. the $n$-dimensional representation $\mathbb{C}^{n}$ with $S_{n}$ acting by $g . e_{j}=e_{g(j)}$ on the standard basis $e_{1}, \ldots, e_{n}$ of $\mathbb{C}^{n}$.

- $\mathfrak{h}_{0}$ (in context of representations of $S_{n}$ ) will denote the reflection representation of the symmetric group $S_{n}$ : this is the $(n-1)$-dimensional irreducible representation of $S_{n}$ corresponding to the partition $(n-1,1)$ of $n$. This representation is the subrepresentation of $\mathfrak{h}$ given by the $S_{n}$-invariant subspace of all vectors whose sum of coordinates is zero.

We will use the same notation for the corresponding object in $\operatorname{Rep}\left(S_{\nu}\right)$.

- Let $s \in \mathbb{Z}_{\geq-1}, k \in \mathbb{Z}_{\geq 0}$. We will denote by $\tau^{s}$ a Young diagram consisting of a row with $s+1$ cells, and by $\pi^{k}$ a Young diagram consisting of a column with $k$ cells.

2.2. Monoidal categories. In the rigid symmetric monoidal category $\underline{\operatorname{Rep}}\left(S_{\nu}\right)$, we will use the following notation:

- $\mathbb{1}$ denotes the unit object.

- For any $U \in \operatorname{Rep}\left(S_{\nu}\right), e v_{U}: U \otimes U^{*} \longrightarrow \mathbb{1}$ is the evaluation morphism, and coev $_{U}: \mathbb{1} \longrightarrow \overline{U^{*}} \otimes U$ is the coevaluation morphism.

- For any $U, U^{\prime} \in \underline{\operatorname{Rep}}\left(S_{\nu}\right), c_{U, U^{\prime}}: U \otimes U^{\prime} \longrightarrow U^{\prime} \otimes U$ is the symmetry isomorphism in $\underline{\operatorname{Rep}}\left(S_{\nu}\right)$.

Notation 2.2.1. Consider a tensor product $V_{1} \otimes V_{2} \otimes \cdots \otimes V_{n}$ (of objects in a symmetric monoidal category) and a sequence $1 \leq i_{1}<\cdots<i_{k} \leq n$. Denote by $V_{i_{1}, i_{2}, \ldots, i_{k}}$ the tensor product $V_{i_{1}} \otimes V_{i_{2}} \otimes \cdots \otimes V_{i_{k}}$. Let $\Omega \in \operatorname{End}\left(V_{i_{1}, i_{2}, \ldots, i_{k}}\right)$.

We write

$$
\Omega^{i_{1}, \ldots, i_{k}} \in \operatorname{End}\left(V_{1} \otimes V_{2} \otimes \cdots \otimes V_{n}\right)
$$

to denote the endomorphism which acts as $\Omega$ on the tensor product of factors $i_{1}, \ldots, i_{k}$, and as Id on the rest. Namely, $\Omega^{i_{1}, \ldots, i_{k}}$ corresponds to the endomorphism

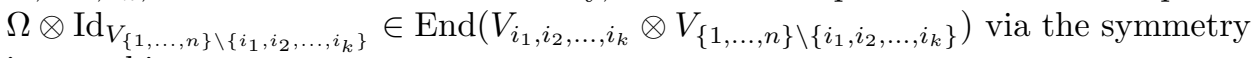
isomorphism

$$
V_{1} \otimes V_{2} \otimes \cdots \otimes V_{n} \cong V_{i_{1}, i_{2}, \ldots, i_{k}} \otimes V_{\{1, \ldots, n\} \backslash\left\{i_{1}, i_{2}, \ldots, i_{k}\right\}} .
$$

Here is an example: given vector spaces $U_{1}, U_{2}, \ldots, U_{5}$ and an operator $A \in$ $\operatorname{End}\left(U_{2} \otimes U_{4} \otimes U_{5}\right)$, the endomorphism $A^{2,4,5} \in \operatorname{End}\left(U_{1} \otimes U_{2} \otimes \cdots \otimes U_{5}\right)$ would be the composition

$$
\begin{aligned}
U_{1} \otimes \cdots \otimes U_{5} & \stackrel{\sim}{\rightarrow} U_{2} \otimes U_{4} \otimes U_{5} \otimes U_{1} \otimes U_{3} \stackrel{A \otimes \operatorname{Id}_{U_{1} \otimes U_{3}}}{\longrightarrow} U_{2} \otimes U_{4} \otimes U_{5} \otimes U_{1} \otimes U_{3} \\
& \stackrel{\sim}{\longrightarrow} U_{1} \otimes \cdots \otimes U_{5} .
\end{aligned}
$$

2.3. Blocks in Karoubian categories. The following definition of a block in a Karoubian category was given in [5] (we will mostly use this notion in the context of abelian categories): 
Definition 2.3.1 (Block of a Karoubian category). Let $\mathcal{C}$ be a Karoubian category. Consider the weakest equivalence relation on the set of isomorphism classes of indecomposable objects such that any two indecomposable objects with a non-zero morphism between them are equivalent.

An equivalence class in this relation will be called a block.

We will use the same term to refer to a full subcategory in $\mathcal{C}$ whose objects are direct sums of indecomposable objects from a single block (in the above sense).

\section{Classical Cherednik algebra of type A}

This section follows [10, Chapter 2], [11, Section 2].

3.1. Rational Cherednik algebra of type A. Let $c \in \mathbb{C}$ (can be considered as a formal parameter).

Definition 3.1.1. The rational Cherednik algebra of type A of rank $n$ with parameter $c$, denoted by $\bar{H}_{c}(n)$, is the quotient of the algebra $\mathbb{C} S_{n} \ltimes T\left(\mathfrak{h} \oplus \mathfrak{h}^{*}\right)$ by the ideal generated by the following relations:

(1) $\left[x, x^{\prime}\right]=0,\left[y, y^{\prime}\right]=0$ for any $x, x^{\prime} \in \mathfrak{h}^{*}, y, y^{\prime} \in \mathfrak{h}$,

(2) $[y, x]=(y, x)-c \sum_{s \in S}((1-s) y, x) s$, where $x \in \mathfrak{h}^{*}, y \in \mathfrak{h},($,$) is the natural$ pairing between $\mathfrak{h}, \mathfrak{h}^{*}$.

Remark 3.1.2. The last relation can also be rewritten as

$$
[y, x]=(y, x)-\frac{c}{2} \sum_{s \in S}((1-s) y,(1-s) x) s .
$$

Both forms of the relation can be interpolated to complex rank; we choose the first one since it is more convenient to interpolate it to complex rank.

If we choose dual bases $x_{1}, \ldots, x_{n}$ of $\mathfrak{h}^{*}, y_{1}, \ldots, y_{n}$ of $\mathfrak{h}$ such that $\forall i, j,\left(y_{j}, x_{i}\right)=$ $\delta_{i j}$, and $s_{i j}\left(y_{i}\right)=y_{j}, s_{i j}\left(x_{i}\right)=x_{j}$, then the last relation from Definition 3.1.1 can be rewritten as

$$
\left[y_{j}, x_{i}\right]=c s_{i j} \text { for any } i \neq j,\left[y_{i}, x_{i}\right]=1-c \sum_{j \neq i} s_{i j} .
$$

Similarly to the universal enveloping algebra of a Lie algebra, we have:

Proposition 3.1.3 (PBW-type theorem). $\bar{H}_{c}(n) \cong S \mathfrak{h}^{*} \otimes \mathbb{C} S_{n} \otimes S \mathfrak{h}$ as vector spaces.

Continuing the analogy between the universal enveloping algebra of a Lie algebra and a Cherednik algebra, we have a Cartan-type element:

Definition 3.1.4. The element $\overline{\boldsymbol{h}} \in \bar{H}_{c}(n)$ is defined as

$$
\overline{\boldsymbol{h}}:=\sum x_{i} y_{i}+\operatorname{dim}(\mathfrak{h}) / 2-c \sum_{s \in S} s=\sum x_{i} y_{i}+n / 2-c \sum_{s \in S} s .
$$

This element satisfies $g \overline{\boldsymbol{h}} g^{-1}=\overline{\boldsymbol{h}},\left[\overline{\boldsymbol{h}}, x_{i}\right]=x_{i},\left[\overline{\boldsymbol{h}}, y_{i}\right]=-y_{i}$ for any $g \in S_{n}, i, j \in$ $\{1, \ldots, n\}$, and can also be written as $\overline{\boldsymbol{h}}=\frac{1}{2} \sum_{i}\left(x_{i} y_{i}+y_{i} x_{i}\right)$.

We will use the "reduced" Cherednik algebra $H_{c}(n)$, which corresponds to the reflection representation $\mathfrak{h}_{0}=\operatorname{span}_{\mathbb{C}}\left\{y_{i}-y_{j} \mid i, j \in\{1, \ldots, n\}, i \neq j\right\}$ rather than the permutation representation $\mathfrak{h}$ of $S_{n}$. It is defined in the same way as $\bar{H}_{c}(n)$, but with $\mathfrak{h}_{0}$ appearing instead of $\mathfrak{h}$ (see [10, or [14, Section 2]). In fact, we have 
$\bar{H}_{c}(n)=H_{c}(n) \otimes \mathbb{A}_{1}, \mathbb{A}_{1}$ being the Weyl algebra. This follows from the fact that the subalgebra of $\bar{H}_{c}(n)$ generated by $\sum y_{i}, \sum x_{i}$ is isomorphic to $\mathbb{A}_{1}$, and commutes with the subalgebra $H_{c}(n)$ of $\bar{H}_{c}(n)$.

For $H_{c}(n), \overline{\boldsymbol{h}}$ is not an element of $H_{c}(n)$, but the element

$$
\boldsymbol{h}:=\overline{\boldsymbol{h}}-\frac{\left(\sum x_{i}\right)\left(\sum y_{i}\right)+\left(\sum y_{i}\right)\left(\sum x_{i}\right)}{2 n}
$$

of $H_{c}(n)$ plays the analogous role.

Remark 3.1.5. Note that the relation between the algebras $H_{c}(n), \bar{H}_{c}(n)$ is similar to the relation between the Lie algebras $\mathfrak{s l}_{n}, \mathfrak{g l}_{n}$.

3.2. Category $\mathcal{O}\left(H_{c}(n)\right)$. The category $\mathcal{O}\left(H_{c}(n)\right)$ is defined as the category of all modules over $H_{c}(n)$ which are finitely generated over $S \mathfrak{h}_{0}^{*}$, and on which $\mathfrak{h}_{0}$ acts locally nilpotently.

3.3. Verma modules. Let $\tau$ be an irreducible representation of $S_{n}$. Then one can define $M_{c, n}(\tau):=H_{c}(n) \otimes_{\mathbb{C} S_{n} \ltimes S \mathfrak{h}_{0}} \tau$, where the augmentation ideal of $S \mathfrak{h}_{0}$ acts on $\tau$ by zero. This is the Verma module corresponding to $\tau$. It is a lowest weight module whose underlying space is isomorphic to $S \mathfrak{h}_{0}^{*} \otimes \tau$, where $S_{n}$ acts on each subspace $S^{m} \mathfrak{h}_{0}^{*} \otimes \tau, \mathfrak{h}_{0}^{*}$ acts by multiplication on $S \mathfrak{h}_{0}^{*}$, and $\mathfrak{h}_{0}$ acts according to the commutator relation given in the definition (if $c=0$, then $\mathfrak{h}_{0}$ acts by differentiation on $S \mathfrak{h}_{0}^{*}$ ). The element $\boldsymbol{h}$ acts locally finitely on modules from $\mathcal{O}\left(H_{c}(n)\right)$, with finite dimensional generalized eigenspaces. In particular, $\boldsymbol{h}$ acts semisimply on $M_{c, n}(\tau)$, with lowest eigenvalue

$$
h_{c, n}(\tau)=\operatorname{dim}\left(\mathfrak{h}_{0}\right) / 2-\left.c \sum_{s \in S} s\right|_{\tau}
$$

(the rest of the eigenvalues on $S^{m} \mathfrak{h}_{0}^{*} \otimes \tau, m>0$ being $h_{c, n}(\tau)+m$ ).

3.3.1. Sum of transpositions element. Recall that $\mathbb{C} S_{n}$ has a central element $\Omega:=$ $\sum_{s \in S} s \in \mathbb{C}\left[S_{n}\right]$. This element acts on an irreducible representation parameterized by the Young diagram $\tau$ by the scalar $c t(\tau)=\sum_{(i, j) \in \tau}(j-i)$, called "content of $\tau$ " (here $(i, j)$ denotes cell in row $i$, column $j$ of the Young diagram $\tau$ ).

So

$$
h_{c, n}(\tau)=\operatorname{dim}\left(\mathfrak{h}_{0}\right) / 2-\left.c \sum_{s \in S} s\right|_{\tau}=\operatorname{dim}\left(\mathfrak{h}_{0}\right) / 2-\left.c \Omega\right|_{\tau}=\frac{n-1}{2}-c \cdot c t(\tau)
$$

\section{Category $\underline{\operatorname{Rep}}\left(S_{\nu}\right)$}

4.1. General description. We recall some basic facts about Deligne's category $\underline{\operatorname{Rep}}\left(S_{\nu}\right)$ (see [6], 9], and [20, Section 2]).

This is a family of Karoubian rigid symmetric monoidal categories over $\mathbb{C}$ defined for any $\nu \in \mathbb{C}$, and "flat with respect to parameter $\nu$ " (one can view $\nu$ as a formal parameter, with $\operatorname{Obj}\left(\underline{\operatorname{Rep}}\left(S_{\nu}\right)\right)$ not depending on $\nu$, and the Hom spaces being modules over $\mathbb{C}((\nu)))$.

For any $\nu \in \mathbb{C}$, the category $\operatorname{Rep}\left(S_{\nu}\right)$ is generated, as a Karoubian tensor category, by one object, denoted $\mathfrak{h}$. This object is the analogue of the permutation representation of $S_{n}$, and any object in $\operatorname{Rep}\left(S_{\nu}\right)$ is a direct summand in a direct sum of tensor powers of $\mathfrak{h}$. 
Remark 4.1.1. Since $\underline{\operatorname{Rep}}\left(S_{\nu}\right)$ is a rigid symmetric monoidal category, the notion of dimension of an object is defined. By definition, the dimension of the object $\mathfrak{h}$ is $\nu$.

For $\nu \notin \mathbb{Z}_{+}, \underline{\operatorname{Rep}}\left(S_{\nu}\right)$ is a semisimple abelian category.

For $\nu \in \mathbb{Z}_{+}$, the category $\operatorname{Rep}\left(S_{\nu}\right)$ has a tensor ideal $\mathfrak{I}_{\nu}$, called the ideal of negligible morphisms (this is the ideal of morphisms $f: X \longrightarrow Y$ such that $\operatorname{tr}(f u)=$ 0 for any morphism $u: Y \longrightarrow X)$. In that case, the classical category $\operatorname{Rep}\left(S_{n}\right)$ of finite-dimensional representations of the symmetric group for $n:=\nu$ is equivalent to $\operatorname{Rep}\left(S_{\nu}\right) / \mathfrak{I}_{\nu}$ (equivalent as Karoubian rigid symmetric monoidal categories). Note $\overline{\text { that }} \mathfrak{h}$ is sent to the permutation representation of $S_{n}$ under this equivalence.

Remark 4.1.2. Although $\operatorname{Rep}\left(S_{\nu}\right)$ is not semisimple and not even abelian when $\nu \in \mathbb{Z}_{+}$, a weaker statement holds (see [6, Proposition 5.1]): consider the full subcategory $\underline{\operatorname{Rep}}\left(S_{\nu}\right)^{(\nu / 2)}$ of $\underline{\operatorname{Rep}}\left(S_{\nu}\right)$ whose objects are direct summands of sums of $\mathfrak{h}^{\otimes m}, 0 \leq \overline{m \leq} \frac{\nu}{2}$. This subcategory is abelian semisimple.

Notation 4.1.3. We will denote Deligne's category for integer value $n \geq 0$ of $\nu$ as $\operatorname{Rep}\left(S_{\nu=n}\right)$, to distinguish it from the classical category $\operatorname{Rep}\left(S_{n}\right)$ of representations of the symmetric group $S_{n}$. Similarly, for other categories arising in this text.

The indecomposable objects of $\operatorname{Rep}\left(S_{\nu}\right)$, regardless of the value of $\nu$, are labeled by all Young diagrams (of arbitrary size). We will denote the indecomposable object in $\operatorname{Rep}\left(S_{\nu}\right)$ corresponding to the Young diagram $\tau$ by $X_{\tau}$.

Notation 4.1.4. We will denote by $\tilde{\lambda}(n)$ the Young diagram obtained from $\lambda$ by adding a top row of size $n-|\lambda|$, where $n \geq \lambda_{1}+|\lambda|$ is an integer.

\section{Example 4.1.5.}

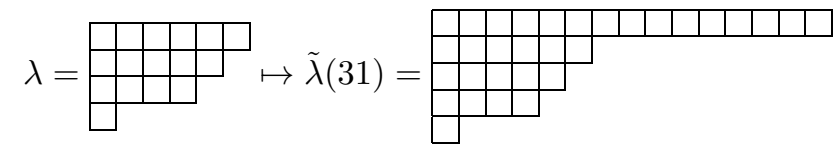

For non-negative integer $\nu=: n$, we have: the partitions $\lambda$ for which $X_{\lambda}$ has a non-zero image in the quotient $\underline{\operatorname{Rep}}\left(S_{\nu=n}\right) / \mathfrak{I}_{\nu=n} \cong \operatorname{Rep}\left(S_{n}\right)$ are exactly the $\lambda$ for which $\lambda_{1}+|\lambda| \leq n$.

If $\lambda_{1}+|\lambda| \leq n$, then the image of $X_{\lambda} \in \underline{\operatorname{Rep}}\left(S_{\nu=n}\right)$ in $\operatorname{Rep}\left(S_{n}\right)$ would be the irreducible representation of $S_{n}$ corresponding to the Young diagram $\tilde{\lambda}(n)$.

Intuitively, one can treat the indecomposable objects of $\operatorname{Rep}\left(S_{\nu}\right)$ as if they were parameterized by "Young diagrams with very long top row". The indecomposable object $X_{\lambda}$ would be treated as if it corresponded to $\tilde{\lambda}(\nu)$, i.e. a Young diagram obtained by adding a very long top row ("of size $\nu-|\lambda|$ "). This point of view is useful to understand how to extend constructions for $S_{n}$ involving Young diagrams to $\underline{\operatorname{Rep}}\left(S_{\nu}\right)$.

Example 4.1.6. The indecomposable object $X_{\lambda}$, where

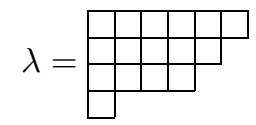

can be thought of as a Young diagram with a "very long top row of length $(\nu-16)$ ":

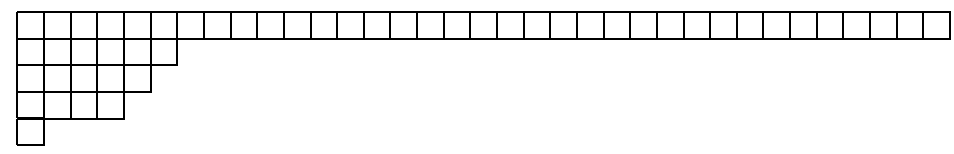


Notation 4.1.7. Let $\mathfrak{h}_{0}:=X_{\square}$ be the indecomposable object in $\underline{\operatorname{Rep}}\left(S_{\nu}\right)$ corresponding to the one-box Young diagram (that would be the analogue of the reflection representation in $\operatorname{Rep}\left(S_{n}\right)$ ).

Its dual in $\underline{\operatorname{Rep}}\left(S_{\nu}\right)$, denoted by $\mathfrak{h}_{0}^{*}$, is isomorphic to $\mathfrak{h}_{0}$, since all the objects in $\underline{\operatorname{Rep}}\left(S_{\nu}\right)$ have a symmetric form defined on them (analogue of the invariant symmet-

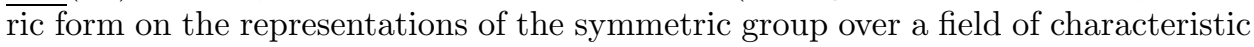
zero).

Remark 4.1.8. $\mathfrak{h} \cong \mathfrak{h}_{0} \oplus \mathbb{1}$ for any $\nu \neq 0$, in particular, for any $\nu \notin \mathbb{Z}_{+}$. But for $\nu=0$, we have $\mathfrak{h} \cong \mathfrak{h}_{0}$. Computing the dimension of $\mathfrak{h}_{0}$, we conclude that $\operatorname{dim}_{\underline{\operatorname{Rep}}\left(S_{\nu}\right)}\left(\mathfrak{h}_{0}\right)=\nu-1$ when $\nu \neq 0$, and $\operatorname{dim}_{\underline{\operatorname{Rep}}\left(S_{\nu}\right)}\left(\mathfrak{h}_{0}\right)=0$ if $\nu=0$.

Example 4.1.9. $\Lambda^{k}\left(\mathfrak{h}_{0}\right)=X_{\pi^{k}}$ for any $k \geq 0$; in particular, $X_{\emptyset}=\mathbb{1}$.

4.2. Pieri's rule. We have an interpolation of Pieri's rule (see [5. Proposition 5.15], [9], 6] concerning the Pieri rule in the Deligne categories $\underline{\operatorname{Rep}}\left(S_{\nu}\right)$, and [12, Par. 4.3] for Pieri's rule for representations of $S_{n}$ ):

Proposition 4.2.1 (Pieri's rule). Let $\nu \notin \mathbb{Z}_{+}$and let $X_{\tau}$ be a simple object of $\underline{\operatorname{Rep}}\left(S_{\nu}\right)$. As objects of $\underline{\operatorname{Rep}}\left(S_{\nu}\right)$,

$$
\mathfrak{h}_{0} \otimes X_{\tau} \cong \bigoplus_{\mu \in P_{\tau}^{+} \cup P_{\tau}^{-} \cup P_{\tau}^{0}} X_{\mu} \oplus c c(\tau) X_{\tau}
$$

where $P_{\tau}{ }^{+}, P_{\tau}^{-}, P_{\tau}^{0}$ are the sets of all Young diagrams obtained from $\tau$ by adding, deleting, or moving a corner cell, respectively (note that $\tau \notin P_{\tau}^{0}$ ), and $c c(\tau)$ is the number of corner cells of $\tau$.

4.3. Analogue of the sum of transpositions element for $\operatorname{Rep}\left(S_{\nu}\right)$. Consider the following function $C_{\nu}: \mathcal{P} \rightarrow \mathbb{C}$ :

$$
C_{\nu}(\mu):=\frac{(\nu-|\mu|)(\nu-|\mu|-1)}{2}-|\mu|+\operatorname{ct}(\mu) .
$$

This function interpolates the function $c t: \mathcal{P} \rightarrow \mathbb{Z}$ in the sense that $\operatorname{ct}(\tilde{\mu}(n))=$ $C_{\nu=n}(\mu)$ for any Young diagram $\mu$ and any $n \in \mathbb{Z}_{\geq|\mu|+\mu_{1}}$.

J. Comes and V. Ostrik defined in [5] an endomorphism $\Omega$ of the identity functor of the category $\operatorname{Rep}\left(S_{\nu}\right)$ for arbitrary $\nu$ (including non-negative integers) such that for any indecomposable object $X_{\mu}$ in $\operatorname{Rep}\left(S_{\nu}\right)$, the morphism $\left(\Omega-C_{\nu}(\mu) \operatorname{Id}_{X_{\mu}}\right)$ is a nilpotent endomorphism of $X_{\mu}$. The endomorphism $\Omega$ is an interpolation of the central element $\Omega \in \mathbb{C}\left[S_{n}\right]$ defined in Subsection 3.3.1.

Remark 4.3.1. For $X_{\mu} \in \underline{\operatorname{Rep}}\left(S_{\nu}\right)$ such that $\nu \notin\left\{0,1, \ldots,|\mu|+\mu_{1}\right\}$, we have:

$$
\operatorname{dim} \operatorname{End}_{\underline{\operatorname{Rep}}\left(S_{\nu}\right)}\left(X_{\mu}\right)=1 .
$$

Since the endomorphism $\left.\Omega\right|_{X_{\mu}}-C_{\nu}(\mu) \operatorname{Id}_{X_{\mu}}$ of $X_{\mu}$ is nilpotent, we conclude that it is zero, and

$$
\left.\Omega\right|_{X_{\mu}}=C_{\nu}(\mu) \operatorname{Id}_{X_{\mu}}=\left(\frac{(\nu-|\mu|)(\nu-|\mu|-1)}{2}-|\mu|+c t(\mu)\right) \operatorname{Id}_{X_{\mu}} .
$$




\section{Category $\underline{\operatorname{Rep}}\left(H_{c}(\nu)\right)$}

Let $c, \nu \in \mathbb{C}$.

Based on the definition of the category $\operatorname{Rep}\left(S_{\nu}\right)$ defined by Deligne, P. Etingof defined in $\left[9\right.$ the category $\operatorname{Rep}\left(H_{c}(\nu)\right)$ :

Definition 5.0.2 (Category $\left.\operatorname{Rep}\left(H_{c}(\nu)\right)\right)$. $\operatorname{Rep}\left(H_{c}(\nu)\right)$ is the category whose objects are triples $(M, x, y)$, where $M$ is an ind-object of Deligne's category $\underline{\operatorname{Rep}}\left(S_{\nu}\right)$, and

$$
\begin{gathered}
x: \mathfrak{h}_{0}^{*} \otimes M \longrightarrow M, \\
y: \mathfrak{h}_{0} \otimes M \rightarrow M,
\end{gathered}
$$

are morphisms in $\operatorname{Rep}\left(S_{\nu}\right)$ satisfying the conditions:

(1) The morphism

$$
x \circ(\mathrm{Id} \otimes x) \circ\left(\left(\mathrm{Id}-c_{\mathfrak{h}_{0}^{*}, \mathfrak{h}_{0}^{*}}\right) \otimes \mathrm{Id}\right): \mathfrak{h}_{0}^{*} \otimes \mathfrak{h}_{0}^{*} \otimes M \longrightarrow M
$$

is 0 .

(2) The morphism

$$
y \circ(\operatorname{Id} \otimes y) \circ\left(\left(\mathrm{Id}-c_{\mathfrak{h}_{0}, \mathfrak{h}_{0}}\right) \otimes \mathrm{Id}\right): \mathfrak{h}_{0} \otimes \mathfrak{h}_{0} \otimes M \longrightarrow M
$$

is 0 .

(3) The morphism

$$
y \circ(\operatorname{Id} \otimes x)-x \circ(\operatorname{Id} \otimes y) \circ\left(c_{\mathfrak{h}_{0}, \mathfrak{h}_{0}^{*}} \otimes \operatorname{Id}\right): \mathfrak{h}_{0} \otimes \mathfrak{h}_{0}^{*} \otimes M \longrightarrow M
$$

equals

$$
e v_{\mathfrak{h}_{0}} \otimes \operatorname{Id}-c\left(e v_{\mathfrak{h}_{0}} \otimes \mathrm{Id}\right) \circ\left(\Omega^{3}-\Omega^{1,3}\right) .
$$

Here

- $\sigma$ is the symmetry isomorphism in $\operatorname{Rep}\left(S_{\nu}\right)$ (i.e. $c_{U, U^{\prime}}: U \otimes U^{\prime} \longrightarrow U^{\prime} \otimes U$ is the canonical isomorphism in $\operatorname{Rep}\left(\overline{\left.S_{\nu}\right)}\right.$, where $U, U^{\prime}$ are objects of $\left.\operatorname{Rep}\left(S_{\nu}\right)\right)$,

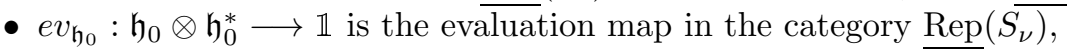

- $\Omega$ is the endomorphism of the identity functor of $\underline{\operatorname{Rep}}\left(S_{\nu}\right) \overline{\operatorname{described}}$ in 4.3 . and the notation $(\cdot)^{3},(\cdot)^{1,3}$ is defined in 2.2.1.

Morphisms in $\underline{\operatorname{Rep}}\left(H_{c}(\nu)\right)$ are defined to be morphisms in ind $-\underline{\operatorname{Rep}}\left(S_{\nu}\right)$ compatible with maps $x, y$.

This category corresponds to the category $\operatorname{Rep}\left(H_{c}(n)\right)$ of representations of the reduced rational Cherednik algebra. One can similarly define the Deligne category $\operatorname{Rep}\left(\bar{H}_{c}(\nu)\right)$ corresponding to the category $\operatorname{Rep}\left(\bar{H}_{c}(n)\right)$ of representations of the non-reduced rational rational Cherednik algebra.

As in the classical case, the relation between the categories $\underline{\operatorname{Rep}}\left(H_{c}(\nu)\right)$ and $\underline{\operatorname{Rep}}\left(\bar{H}_{c}(\nu)\right)$ will be similar to the relation between the categories $\operatorname{Rep}\left(\mathfrak{s l}_{n}\right), \operatorname{Rep}\left(\mathfrak{g l}_{n}\right)$.

\section{Category $\underline{\mathcal{O}}_{c, \nu}$}

6.1. Definition of $\underline{\mathcal{O}}_{c, \nu}$. Consider the algebra objects $S \mathfrak{h}_{0}^{*}, S \mathfrak{h}_{0}$ in ind $-\underline{\operatorname{Rep}}\left(S_{\nu}\right)$.

Given maps $x, y$ as above, we obtain the maps

$$
\begin{gathered}
\bar{x}_{M}: S \mathfrak{h}_{0}^{*} \otimes M \longrightarrow M, \\
\bar{y}_{M}: S \mathfrak{h}_{0} \otimes M \rightarrow M .
\end{gathered}
$$

So we can speak of $M$ as an $S \mathfrak{h}_{0}^{*}$-module and an $S \mathfrak{h}_{0}$-module in ind $-\underline{\operatorname{Rep}}\left(S_{\nu}\right)$. 
Definition 6.1.1. The category $\underline{\mathcal{O}}_{c, \nu}$ is defined as the full subcategory of $\underline{\operatorname{Rep}}\left(H_{c}(\nu)\right)$ whose objects are $(M, x, y)$ such that

- $M$ is finitely generated over $S \mathfrak{h}_{0}^{*}$ in $\operatorname{Rep}\left(S_{\nu}\right)$ (i.e. $M$ is a quotient of a "finitely generated free $S \mathfrak{h}_{0}^{*}$ module" $\overline{S \mathfrak{h}_{0}^{*}} \otimes V$, where $V$ is an object of $\left.\operatorname{Rep}\left(S_{\nu}\right)\right)$.

- $\bar{M}$ is locally nilpotent over $\mathfrak{h}_{0}$, in the following sense:

for any $\operatorname{Rep}\left(S_{\nu}\right)$-subobject $T \subset M$, there exists a non-negative integer $m$ (depending on $T$ ) such that the map $S^{m} \mathfrak{h}_{0} \otimes T \longrightarrow M$ (this is the restriction to $T$ of the map $S^{m} \mathfrak{h}_{0} \otimes M \rightarrow M$ ) equals zero.

Definition 6.1.2. Define $\boldsymbol{h} \in \operatorname{End}\left(\right.$ Forget $\left._{\underline{\mathcal{O}}_{c, \nu} \rightarrow \operatorname{ind}-\underline{\operatorname{Rep}}\left(S_{\nu}\right)}\right)$ by letting $\boldsymbol{h}_{M}:=$ $\frac{1}{2}\left(\psi_{1}+\psi_{2}\right)$ for every $M \in \underline{\mathcal{O}}_{c, \nu}$, where $\psi_{1}, \psi_{2}: M \longrightarrow \bar{M}$,

$$
\begin{aligned}
& \psi_{1}=x \circ(\operatorname{Id} \otimes y) \circ\left(\operatorname{coev}_{\mathfrak{h}_{0}^{*}} \otimes \operatorname{Id}_{M}\right): M \longrightarrow \mathfrak{h}_{0}^{*} \otimes \mathfrak{h}_{0} \otimes M \longrightarrow M, \\
& \psi_{2}=y \circ(\operatorname{Id} \otimes x) \circ\left(\operatorname{coev}_{\mathfrak{h}_{0}} \otimes \operatorname{Id}_{M}\right): M \longrightarrow \mathfrak{h}_{0} \otimes \mathfrak{h}_{0}^{*} \otimes M \longrightarrow M .
\end{aligned}
$$

Similarly to the classical case, an easy computation shows that $\boldsymbol{h}$ has the following properties:

Lemma 6.1.3. The endomorphism $\boldsymbol{h} \in \operatorname{End}\left(\operatorname{Forget}_{\underline{\mathcal{O}}_{c, \nu} \rightarrow \operatorname{ind}-\operatorname{Rep}\left(S_{\nu}\right)}\right)$ can also be defined by setting $\left.\boldsymbol{h}\right|_{M}: M \rightarrow M$ to be

$$
\left.\boldsymbol{h}\right|_{M}:=x \circ(\operatorname{Id} \otimes y) \circ\left(\operatorname{coev}_{\mathfrak{h}_{0}^{*}} \otimes \operatorname{Id}_{M}\right)+\frac{\operatorname{dim}\left(\mathfrak{h}_{0}\right)}{2}-c \Omega_{M}
$$

(the endomorphism $\Omega$ is defined in Subsection 4.3).

In addition, $\boldsymbol{h}$ satisfies the the following commutation relations:

$$
\begin{aligned}
& y_{M} \circ\left(\operatorname{Id}_{\mathfrak{h}_{0}} \otimes \boldsymbol{h}\right)-\boldsymbol{h} \circ y_{M}=y_{M}: \mathfrak{h}_{0} \otimes M \longrightarrow M, \\
& x_{M} \circ\left(\operatorname{Id}_{\mathfrak{h}_{0}^{*}} \otimes \boldsymbol{h}\right)-\boldsymbol{h} \circ x_{M}=-x_{M}: \mathfrak{h}_{0}^{*} \otimes M \longrightarrow M .
\end{aligned}
$$

Definition 6.1.4 (Indecomposable singular subobject). Let $U \in \underline{\mathcal{O}}_{c, \nu}, V \in \underline{\operatorname{Rep}}\left(S_{\nu}\right)$ such that $V \subset U$ and the map $y_{U}$ restricted to $\mathfrak{h}_{0} \otimes V$ is zero. Then $V$ is called "singular in $U$ ". If, furthermore, $V$ is an indecomposable (resp. simple) object in $\underline{\operatorname{Rep}}\left(S_{\nu}\right)$, then we say that $V$ is an indecomposable (resp. simple) singular $\underline{\operatorname{Rep}}\left(S_{\nu}\right)$ subobject of $U$.

Remark 6.1.5. Note that by definition of $\underline{\mathcal{O}}_{c, \nu}$, each object contains an indecomposable singular $\operatorname{Rep}\left(S_{\nu}\right)$-subobject.

6.2. Verma objects in the category $\underline{\mathcal{O}}_{c, \nu}$. One can define Verma objects in the category $\underline{\mathcal{O}}_{c, \nu}$ as follows:

Consider the category $\underline{\operatorname{Rep}}\left(B_{\nu}\right)$ of pairs $\left(M, y_{M}\right)$, where $M$ is an ind-object of $\underline{\operatorname{Rep}}\left(S_{\nu}\right)$,

$$
y: \mathfrak{h}_{0} \otimes M \rightarrow M
$$

is a morphism in $\underline{\operatorname{Rep}}\left(S_{\nu}\right)$ satisfying the following condition: the morphism

$$
y \circ(\operatorname{Id} \otimes y) \circ\left(\left(\mathrm{Id}-c_{\mathfrak{h}_{0}, \mathfrak{h}_{0}}\right) \otimes \mathrm{Id}\right): \mathfrak{h}_{0} \otimes \mathfrak{h}_{0} \otimes M \longrightarrow M
$$

is zero. The morphisms in $\underline{\operatorname{Rep}}\left(B_{\nu}\right)$ are morphisms of $\underline{\operatorname{Rep}}\left(S_{\nu}\right)$ ind-objects compatible with the $y_{M}$ maps.

This is the analogue of the category of representations of the "Borel" subalgebra $B_{n}=\mathbb{C}\left[S_{n}\right] \ltimes S \mathfrak{h}_{0}$. 
Then we have the restriction functor Res : $\operatorname{Rep}\left(H_{c}(\nu)\right) \longrightarrow \operatorname{Rep}\left(B_{\nu}\right)$, and it has a left adjoint which is the induction functor Ind $: \operatorname{Rep}\left(B_{\nu}\right) \longrightarrow \operatorname{Rep}\left(H_{c}(\nu)\right)$. The induction functor takes a pair $\left(M, y_{M}\right)$ to a triple $\left(\overline{S \mathfrak{h}_{0}^{*} \otimes} M, y_{S \mathfrak{h}_{0}^{*} \otimes M}, \bar{x}_{S \mathfrak{h}_{0}^{*} \otimes M}\right)$, where the map $x_{S \mathfrak{h}_{0}^{*} \otimes M}: \mathfrak{h}_{0}^{*} \otimes S \mathfrak{h}_{0}^{*} \otimes M \rightarrow S \mathfrak{h}_{0}^{*} \otimes M$ is the multiplication map of $\mathfrak{h}_{0}^{*} \otimes S \mathfrak{h}_{0}^{*} \rightarrow S \mathfrak{h}_{0}^{*}$ tensored with $\mathrm{Id}_{M}$, and $y_{S \mathfrak{h}_{0}^{*} \otimes M}: \mathfrak{h}_{0} \otimes S \mathfrak{h}_{0}^{*} \otimes M \rightarrow S \mathfrak{h}_{0}^{*} \otimes M$ is the unique map satisfying conditions (1) and (3) of Definition 5.0.2, such that $\left.y_{S \mathfrak{h}_{0}^{*} \otimes M}\right|_{\mathfrak{h}_{0} \otimes 1 \otimes M}=y_{M}$.

Definition 6.2.1 (Verma object). Consider an indecomposable object $X_{\tau}$ in $\operatorname{Rep}\left(S_{\nu}\right)$, and let the map $y_{X_{\tau}}: \mathfrak{h}_{0} \otimes X_{\tau} \rightarrow X_{\tau}$ be zero. This makes $\left(X_{\tau}, y_{X_{\tau}}\right)$ an object of $\operatorname{Rep}\left(B_{\nu}\right)$.

Define the Verma object of lowest weight $X_{\tau}$ as $\operatorname{Ind}\left(X_{\tau}, y_{X_{\tau}}\right)$ (so it is an indobject of $\operatorname{Rep}\left(S_{\nu}\right)$ ). It will be denoted by $M_{c, \nu}(\tau)$ (or, if $c, \nu$ are fixed, $M(\tau)$ for short).

Observation 6.2.2. It is easy to see that as a $\operatorname{Rep}\left(S_{\nu}\right)$ ind-object $M_{c, \nu}(\tau)=$ $S \mathfrak{h}_{0}^{*} \otimes X_{\tau}$, where $X_{\tau}$ is an indecomposable object of $\operatorname{Rep}\left(S_{\nu}\right)$.

The map $\bar{x}_{M_{c, \nu}(\tau)}: S \mathfrak{h}_{0}^{*} \otimes S \mathfrak{h}_{0}^{*} \otimes X_{\tau} \longrightarrow S \mathfrak{h}_{0}^{*} \otimes X_{\tau}$ is the multiplication map of $S \mathfrak{h}_{0}^{*}$ tensored with $\operatorname{Id}_{X_{\tau}}$.

The map $\bar{y}_{M_{c, \nu}(\tau)}: S \mathfrak{h}_{0} \otimes S \mathfrak{h}_{0}^{*} \otimes X_{\tau} \longrightarrow S \mathfrak{h}_{0}^{*} \otimes X_{\tau}$ is the map for which condition (3) from Definition 5.0 .2 holds, and thus is a deformation of the differentiation map $S \mathfrak{h}_{0} \otimes S \mathfrak{h}_{0}^{*} \longrightarrow S \mathfrak{h}_{0}^{*}$ tensored with $\operatorname{Id}_{X_{\tau}}$.

Notation 6.2.3. To avoid confusion, the Verma module in $\mathcal{O}\left(H_{c}(n)\right)$ whose lowest weight corresponds to the Young diagram $\tilde{\tau}(n)$ is denoted by $M_{c, n}(\tilde{\tau}(n))$, while the Verma object in $\underline{\mathcal{O}}_{c, \nu=n}$ corresponding to the Young diagram $\tau$ is denoted by $M_{c, \nu=n}(\tau)$.

Definition 6.2.4. Let $c, \nu \in \mathbb{C}$. We define the function $h_{c, \nu}: \mathcal{P} \rightarrow \mathbb{C}$ as $h_{c, \nu}(\tau):=\frac{\operatorname{dim}\left(\mathfrak{h}_{0}\right)}{2}-c \cdot C_{\nu}(\tau)=\frac{\nu-1}{2}-c \cdot\left(\frac{(\nu-|\tau|)(\nu-|\tau|-1)}{2}-|\tau|+\operatorname{ct}(\tau)\right)$.

We immediately see that this function interpolates the functions $h_{c, n}$ defined in Subsection 3.3.1. Namely, $h_{c, n}(\tilde{\tau}(n))=h_{c, \nu=n}(\tau)$ for large enough $n$.

Remark 6.2.5. Note that given $X_{\tau} \in \underline{\operatorname{Rep}}\left(S_{\nu}\right)$ such that $\nu \notin\left\{0,1, \ldots,|\tau|+\tau_{1}\right\}$, we have

$$
h_{c, \nu}(\tau) \operatorname{Id}_{X_{\tau}}=\frac{\operatorname{dim}\left(\mathfrak{h}_{0}\right)}{2} \operatorname{Id}_{X_{\tau}}-\left.c \Omega\right|_{X_{\tau}}
$$

which is an interpolation of the formula in Subsection 3.3. Here $\Omega$ is the endomorphism of the identity functor of $\operatorname{Rep}\left(S_{\nu}\right)$ described in Subsection 4.3. and identity follows from Remark 4.3.1.

\section{Example 6.2.6.}

$$
\begin{aligned}
& \text { - } c t\left(\tau^{s-1}\right)=\frac{s(s-1)}{2}, \text { so } h_{c, \nu}\left(\tau^{s}\right)=\frac{\operatorname{dim}\left(\mathfrak{h}_{0}\right)}{2}-c \cdot\left(\frac{\nu^{2}-\nu}{2}-s \nu+s^{2}-s\right) . \\
& \text { - } c t\left(\pi^{n}\right)=-\frac{n(n-1)}{2}, \text { so } h_{c, \nu}\left(\pi^{n}\right)=\frac{\operatorname{dim}\left(\mathfrak{h}_{0}\right)}{2}-c \cdot\left(\frac{\nu^{2}-\nu}{2}-n \nu\right) .
\end{aligned}
$$

We now consider the action of the endomorphism $\boldsymbol{h}$ on Verma objects. 
Proposition 6.2.7. Let $c \in \mathbb{C}$.

The endomorphism $\boldsymbol{h}: M_{c, \nu}(\tau) \longrightarrow M_{c, \nu}(\tau)$ of the ind-object

$$
M_{c, \nu}(\tau)=\bigoplus_{m \geq 0} S^{m} \mathfrak{h}_{0}^{*} \otimes X_{\tau}
$$

of $\underline{\operatorname{Rep}}\left(S_{\nu}\right)$ decomposes as a sum

$$
\boldsymbol{h}=\bigoplus_{m \geq 0} \phi_{m}
$$

where $\phi_{m} \in \operatorname{End}\left(S^{m} \mathfrak{h}_{0}^{*} \otimes X_{\tau}\right)$ for every $m \geq 0$. Furthermore,

$$
\phi_{0}=\frac{\operatorname{dim}\left(\mathfrak{h}_{0}\right)}{2} \operatorname{Id}_{X_{\tau}}-\left.c \Omega\right|_{X_{\tau}}
$$

and the endomorphism $\left(\phi_{m}-\left(h_{c, \nu}(\tau)+m\right) \operatorname{Id}_{X_{\tau}}\right)$ of $S^{m} \mathfrak{h}_{0}^{*} \otimes X_{\tau}$ is nilpotent for every $m \geq 0$. In particular, if $\nu \notin\left\{0,1, \ldots,|\tau|+\tau_{1}\right\}$, then

$$
\phi_{m}=\left(h_{c, \nu}(\tau)+m\right) \operatorname{Id}_{S^{m} \mathfrak{h}_{0}^{*} \otimes X_{\tau}}
$$

for any $m \geq 0$.

Proof. From the definition of $\boldsymbol{h}$ (Definition 6.1.2) and Observation 6.2.2, we immediately obtain a decomposition $\boldsymbol{h}=\bigoplus_{m>0} \phi_{m}$ where $\phi_{m} \in \operatorname{End}\left(S^{m} \mathfrak{h}_{0}^{*} \otimes X_{\tau}\right)$ (this amounts to checking that $\boldsymbol{h}$ acts by operators of degree zero on the graded space $S \mathfrak{h}_{0}^{*} \otimes X_{\tau}$, with the $\mathbb{Z}_{+}$-grading inherited from $\left.S \mathfrak{h}_{0}^{*}\right)$.

We now want to prove that the endomorphism $\left(\phi_{m}-\left(h_{c, \nu}(\tau)+m\right) \operatorname{Id}_{X_{\tau}}\right)$ of $S^{m} \mathfrak{h}_{0}^{*} \otimes X_{\tau}$ is nilpotent.

First, we consider the case $m=0$. By Lemma 6.1.3. the endomorphism $\phi_{0}$ acts on $X_{\tau}$ as the operator $\frac{\operatorname{dim}\left(\mathfrak{h}_{0}\right)}{2}-c \Omega_{M}$, which has a unique generalized eigenvalue $h_{c, \nu}(\tau)$, as it was said in Subsection 4.3 .

Next, we prove by induction on $m \geq 0$ that $\phi_{m}$ has only one generalized eigenvalue, which is $h_{c, \nu}(\tau)+m$. Just like in the classical setting, this is a direct consequence of the commutation relation

$$
(\boldsymbol{h}-(a+1))^{N} \circ x_{M_{c, \nu}(\tau)}=x_{M_{c, \nu}(\tau)} \circ\left(\operatorname{Id}_{\mathfrak{h}_{0}^{*}} \otimes(\boldsymbol{h}-a)^{N}\right) .
$$

Here both sides are morphisms $\mathfrak{h}_{0}^{*} \otimes S \mathfrak{h}_{0}^{*} \otimes X_{\tau} \rightarrow S \mathfrak{h}_{0}^{*} \otimes X_{\tau}, a$ is (any) scalar and $N$ is any non-negative integer. This commutation relation is easily obtained from Lemma 6.1.3.

Finally, if $\nu \notin\left\{0,1, \ldots,|\tau|+\tau_{1}\right\}$, then the object $X_{\tau}$ is simple, and thus

$$
\phi_{0}=\frac{\operatorname{dim}\left(\mathfrak{h}_{0}\right)}{2} \operatorname{Id}_{X_{\tau}}-\left.c \Omega\right|_{X_{\tau}}=h_{c, \nu}(\tau) \operatorname{Id}_{X_{\tau}} .
$$

Due to the commutation relation above, we conclude that

$$
\phi_{m}=\left(h_{c, \nu}(\tau)+m\right) \operatorname{Id}_{S^{m} \mathfrak{h}_{0}^{*} \otimes X_{\tau}}
$$

for any $m \geq 0$.

This proposition means that $M(\tau)$ (as a $\underline{\operatorname{Rep}}\left(S_{\nu}\right)$ ind-object) has a grading by eigenvalues of $\boldsymbol{h}$, which is the natural $\mathbb{Z}_{+}$-grading on $M(\tau) \cong S \mathfrak{h}_{0}^{*} \otimes X_{\tau}$ shifted by $h_{c, \nu}(\tau)$.

Now let $U$ be a subquotient of $M(\tau)$. Then $U$ automatically inherits a grading from the grading of $M(\tau)$ by eigenvalues of $\boldsymbol{h}$, and the definition of $\boldsymbol{h}$ implies that maps between subquotients of Verma objects preserve this grading. 
For simplicity, we will use the natural $\mathbb{Z}_{+}$-grading on $M(\tau) \cong S \mathfrak{h}_{0}^{*} \otimes X_{\tau}$ when we refer to the degree in which a $\operatorname{Rep}\left(S_{\nu}\right)$-object lies in $U$.

Proposition 6.2.8. Let $U \in \underline{\mathcal{O}}_{c, \nu}$. Let $X_{\mu}$ be an indecomposable singular $\operatorname{Rep}\left(S_{\nu}\right)$ subobject in $U$. Then there exists a morphism $M_{c, \nu}(\mu) \longrightarrow U$ in $\underline{\mathcal{O}}_{c, \nu}$, inducing $\operatorname{Id}_{X_{\mu}}$ on $X_{\mu}$ when regarded as a morphism of $\underline{\operatorname{Rep}}\left(S_{\nu}\right)$ ind-objects.

Proof. Recall the definition of a Verma object. $X_{\mu}$ being an indecomposable singular $\operatorname{Rep}\left(S_{\nu}\right)$-subobject in $U$ means that $\operatorname{Hom}_{\operatorname{Rep}\left(B_{\nu}\right)}\left(X_{\mu}, \operatorname{Res}(U)\right) \neq 0$, where $X_{\mu}$ is considered as a $\operatorname{Rep}\left(B_{\nu}\right)$-object with $y_{X_{\mu}}=0$, and there exists a nonzero map in $\left.\operatorname{Hom}_{\operatorname{Rep}\left(B_{\nu}\right)} \overline{(X}_{\mu}, \operatorname{Res}(U)\right)$ whose image is the chosen copy of $X_{\mu}$ in $U$. Then by definition of a Verma object, we have: $\operatorname{Hom}_{\operatorname{Rep}\left(B_{\nu}\right)}\left(X_{\mu}, \operatorname{Res}(U)\right)=$ $\operatorname{Hom}_{\underline{\mathcal{O}}_{c, \nu}}\left(M_{c, \nu}(\mu), U\right) \neq 0$ and there exists a non-zero map in $\operatorname{Hom}_{\underline{\mathcal{O}}_{c, \nu}}\left(M_{c, \nu}(\mu), U\right)$ inducing $\operatorname{Id}_{X_{\mu}}$ on $X_{\mu}$ when regarded as a morphism of $\underline{\operatorname{Rep}}\left(S_{\nu}\right)$ ind-objects.

Proposition 6.2.9. Assume $\nu \notin \mathbb{Z}_{+}$. Then

- Each Verma object $M_{c, \nu}(\tau)$ has a maximal proper $\underline{\mathcal{O}}_{c, \nu}$-subobject $J$, i.e. it has a unique simple quotient $L_{c, \nu}(\tau)$ in $\underline{\mathcal{O}}_{c, \nu}$.

- The simple objects of the category $\underline{\mathcal{O}}_{c, \nu}$ are exactly $L_{c, \nu}(\tau)$.

Proof. Consider the natural $\mathbb{Z}_{+}$-grading on $M_{c, \nu}(\tau)$ (as an ind-object of $\operatorname{Rep}\left(S_{\nu}\right)$ ). Recall that by Proposition 6.2.7 $\boldsymbol{h}$ acts on grade $m$ of $M_{c, \nu}(\tau)$ by $\left(h_{c, \nu}(\tau)+m\right)$ Id.

- Let $J$ be the sum of all the proper $\underline{\mathcal{O}}_{c, \nu}$-subobjects of $M_{c, \nu}(\tau)$. For any proper $\underline{\mathcal{O}}_{c, \nu}$-subobject $N$ of $M_{c, \nu}(\tau), N$ inherits the $\mathbb{Z}_{+}$-grading from $M_{c, \nu}(\tau)$, with $\boldsymbol{h}$ acting on grade $m$ of $N$ by $\left(h_{c, \nu}(\tau)+m\right)$ Id. Since $N$ is a proper $S \mathfrak{h}_{0}^{*}$-submodule, we have $m>0$. So $J$ can be presented as a direct sum of $\operatorname{Rep}\left(S_{\nu}\right)$-objects on which the restriction of $\boldsymbol{h}$ acts by diagonally, with eigenvalues whose real part is greater or equal to $h_{c, \nu}(\tau)+1$. This proves that $J$ is a proper $\underline{\mathcal{O}}_{c, \nu}$-subobject of $M_{c, \nu}(\tau)$.

- Let $L$ be a simple object of $\underline{\mathcal{O}}_{c, \nu}$. Then $L$ has a simple singular $\operatorname{Rep}\left(S_{\nu}\right)$ subobject $X_{\tau}$. By Proposition 6.2 .8 , there exists a non-zero map $\overline{M(\tau)} \longrightarrow$ $L$, with induced map on $X_{\tau}$ being $\operatorname{Id}_{\tau}$. But $L$ is simple, so $M(\tau) \longrightarrow L$ is surjective.

Remark 6.2.10. Let $M_{c, \nu}(\tau)$ be a Verma object in $\underline{\mathcal{O}}_{c, \nu}$. By the propositions above, to check its reducibility, we only need to check whether there are any non-zero morphisms from other Verma objects to $M_{c, \nu}(\tau)$.

\section{Functor $\underline{\mathcal{O}}_{c, \nu=n} \rightarrow \mathcal{O}\left(H_{c}(n)\right)$}

In this section we construct a functor $\mathcal{F}_{c, n}: \underline{\operatorname{Rep}}\left(H_{c}(\nu=n)\right) \longrightarrow \operatorname{Rep}\left(H_{c}(n)\right)$ restricting to $\mathcal{F}_{c, n}: \underline{\mathcal{O}}_{c, \nu=n} \longrightarrow \mathcal{O}\left(H_{c}(n)\right)$ for any $c \in \mathbb{C}, n \in \mathbb{Z}_{+}$. This functor is analogous to (and based on) the functor $\mathcal{F}_{n}: \operatorname{Rep}\left(S_{\nu=n}\right) \longrightarrow \operatorname{Rep}\left(S_{n}\right)$ which induces the equivalence $\operatorname{Rep}\left(S_{\nu=n}\right) / \mathfrak{I}_{\nu=n} \cong \operatorname{Rep}\left(S_{n}\right)$ discussed in Section 4 .

The functor $\mathcal{F}_{c, n}$ is constructed as follows: let $(M, x, y) \in \operatorname{Rep}\left(H_{c}(\nu)\right)$ (see Section 5 , and consider $\mathcal{F}_{n}(M) \in$ ind $-\operatorname{Rep}\left(S_{n}\right)$. The maps $\overline{\mathcal{F}_{n}(x)}, \mathcal{F}_{n}(y)$ then make $\mathcal{F}_{n}(M)$ a module over the algebra $\mathbb{C} S_{n} \ltimes T\left(\mathfrak{h}_{0} \oplus \mathfrak{h}_{0}^{*}\right)$, and the conditions on the maps $x, y$ given in Section 5 imply that this action factors through $H_{c}(n)$, thus making $\mathcal{F}_{n}(M)$ an $H_{c}(n)$-module. 
Recall that a morphism $\phi:\left(M_{1}, x_{1}, y_{1}\right) \longrightarrow\left(M_{2}, x_{2}, y_{2}\right)$ in $\operatorname{Rep}\left(H_{c}(\nu)\right)$ is by definition a morphism $\phi: M_{1} \longrightarrow M_{2}$ of $\underline{\operatorname{Rep}}\left(S_{\nu=n}\right)$ ind-objects such that $x_{2} \circ$ $\left(\operatorname{Id}_{\mathfrak{h}^{*}} \otimes \phi\right)=\phi \circ x_{1}$ and $y_{2} \circ\left(\operatorname{Id}_{\mathfrak{h}} \otimes \phi\right)=\phi \circ y_{1} . \overline{\text { So }} \mathcal{F}_{n}(\phi)$ is automatically a morphism of $H_{c}(n)$-modules.

Thus we define

$$
\begin{gathered}
\mathcal{F}_{c, n}(M, x, y):=\mathcal{F}_{n}(M) \text { with action of } H_{c}(n) \text { given by } \mathcal{F}_{n}(x), \mathcal{F}_{n}(y), \\
\mathcal{F}_{c, n}(\phi):=\mathcal{F}_{n}(\phi) .
\end{gathered}
$$

To see that $\mathcal{F}_{c, n}$ restricts to $\underline{\mathcal{O}}_{c, \nu=n} \longrightarrow \mathcal{O}\left(H_{c}(n)\right)$, note that $\mathcal{F}_{n}(S \mathfrak{h})=S \mathfrak{h}$, $\mathcal{F}_{n}\left(S \mathfrak{h}^{*}\right)=S \mathfrak{h}^{*}$. The definitions of $\underline{\mathcal{O}}_{c, \nu=n}, \mathcal{O}\left(H_{c}(n)\right)$ then imply that

$$
\mathcal{F}_{c, n}\left(\underline{\mathcal{O}}_{c, \nu=n}\right) \subset \mathcal{O}\left(H_{c}(n)\right) .
$$

In particular, if $|\lambda|+\lambda_{1} \leq n$ then the Verma object of lowest weight $\lambda$ goes to the Verma module of lowest weight $\tilde{\lambda}(n)$.

Finally, a lemma which will be useful to us later on:

Lemma 7.0.11. Fix Young diagrams $\mu, \tau$, and let $n>>$ (in fact, it is enough that $n>2 \max (|\mu|,|\tau|)+1)$. Then

$\mathcal{F}_{c, n}: \operatorname{Hom}_{\underline{\mathcal{O}}_{c, \nu=n}}\left(M_{c, \nu=n}(\mu), M_{c, \nu=n}(\tau)\right) \rightarrow \operatorname{Hom}_{\mathcal{O}\left(H_{c}(n)\right)}\left(M_{c, n}(\tilde{\mu}(n)), M_{c, n}(\tilde{\tau}(n))\right)$ is a bijection.

Proof. Recall that we have:

$$
\begin{aligned}
& \operatorname{Hom}_{\underline{\mathcal{O}}} c, \nu=n \\
& \cong\left\{\phi \in \operatorname{Hom}_{c, \nu=n}(\mu), M_{c, \nu=n}\left(S_{\nu=n}\right)\right. \\
& \left.\left.\cong X_{\mu}, M_{c, \nu=n}(\tau)\right):\left.y_{M_{c, \nu=n}(\tau)}\right|_{\mathfrak{h}_{0} \otimes \phi\left(X_{\mu}\right)}=0\right\}
\end{aligned}
$$

and

$$
\begin{aligned}
& \operatorname{Hom}_{\mathcal{O}\left(\bar{H}_{c}(n)\right)}\left(M_{c, n}(\tilde{\mu}(n)), M_{c, n}(\tilde{\tau}(n))\right) \\
& \cong\left\{\psi \in \operatorname{Hom}_{R e p\left(S_{n}\right)}\left(\tilde{\mu}(n), M_{c, n}(\tilde{\tau}(n))\right):\left.\mathcal{F}_{c, n}\left(y_{M_{c, \nu=n}(\tau)}\right)\right|_{\mathfrak{h}_{0} \otimes \psi(\tilde{\mu}(n))}=0\right\} .
\end{aligned}
$$

It remains to check that

$$
\begin{aligned}
& \mathcal{F}_{n}:\left\{\phi \in \operatorname{Hom}_{\underline{\operatorname{Rep}}\left(S_{\nu=n}\right)}\left(X_{\mu}, M_{c, \nu=n}(\tau)\right):\left.y_{M_{c, \nu=n}(\tau)}\right|_{\mathfrak{h}_{0} \otimes \phi\left(X_{\mu}\right)}=0\right\} \\
& \longrightarrow\left\{\psi \in \operatorname{Hom}_{\operatorname{Rep}\left(S_{n}\right)}\left(\tilde{\mu}(n), M_{c, n}(\tilde{\tau}(n))\right):\left.\mathcal{F}_{c, n}\left(y_{M_{c, \nu=n}(\tau)}\right)\right|_{\mathfrak{h}_{0} \otimes \psi(\tilde{\mu}(n))}=0\right\}
\end{aligned}
$$

is an isomorphism. Let $\psi \in \operatorname{Hom}_{\operatorname{Rep}\left(S_{n}\right)}\left(\tilde{\mu}(n), M_{c, n}(\tilde{\tau}(n))\right)$ such that

$$
\left.\mathcal{F}_{c, n}\left(y_{M_{c, \nu=n}(\tau)}\right)\right|_{\mathfrak{h}_{0} \otimes \psi(\tilde{\mu}(n))}=0 .
$$

Since $\mathcal{F}_{n}$ is surjective on Hom-spaces, there is a $\phi \in \operatorname{Hom}_{\operatorname{Rep}\left(S_{\nu=n}\right)}\left(X_{\mu}, M_{c, \nu=n}(\tau)\right)$ such that $\mathcal{F}_{n}(\phi)=\psi$. We need to check that there exists a unique such $\phi$ so that $\left.y_{M_{c, \nu=n}(\tau)}\right|_{\mathfrak{h}_{0} \otimes \phi\left(X_{\mu}\right)}=0$.

Let $m \in \mathbb{Z}_{+}$be such that $\psi(\tilde{\mu}(n)) \subset S^{m} \mathfrak{h}_{0}^{*} \otimes \tilde{\tau}(n) \subset M_{c, n}(\tilde{\tau}(n))$. Recall that

$$
\mathcal{F}_{n}\left(\left.y\right|_{\mathfrak{h}_{0} \otimes \phi\left(X_{\mu}\right)}\right)=\left.y_{n}\right|_{\mathfrak{h}_{0} \otimes \tilde{\mu}(n)}=0
$$

so $\left.y\right|_{\mathfrak{h}_{0} \otimes \phi\left(X_{\mu}\right)}: \mathfrak{h}_{0} \otimes X_{\mu} \longrightarrow S^{m} \mathfrak{h}_{0}^{*} \otimes X_{\tau}$ is a negligible morphism in $\underline{\operatorname{Rep}}\left(S_{\nu=n}\right)$.

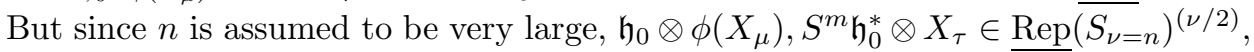
which is a semisimple subcategory of $\operatorname{Rep}\left(S_{\nu=n}\right)$ (see Section 4). So a negligible morphism in this subcategory is zero, and thus $\left.y\right|_{\mathfrak{h}_{0} \otimes \phi\left(X_{\mu}\right)}=0$.

Similarly, the fact that $X_{\mu}, S^{m} \mathfrak{h}_{0}^{*} \otimes X_{\tau} \in \underline{\operatorname{Rep}}\left(S_{\nu=n}\right)^{(\nu / 2)}$ implies that such a morphism $\phi$ is unique. 


\section{Morphisms between Verma objects: General Remarks AND NECESSARY CONDITIONS}

8.1. Necessary conditions. The following formula will serve as one of the main tools of this paper.

Proposition 8.1.1. Let $\mu$ be a partition and $m>0$ an integer, and assume there is a non-zero morphism $\phi: M_{c, \nu}(\mu) \longrightarrow M_{c, \nu}(\tau)$ such that $\phi\left(X_{\mu}\right)$ (image of the lowest weight of $M(\mu))$ sits in degree $m$ of $M_{c, \nu}(\tau)$. Then

$$
c\left(\frac{|\tau|^{2}-|\mu|^{2}-(|\tau|-|\mu|)}{2}+c t(\tau)-c t(\mu)\right)=m+(|\tau|-|\mu|) c \nu .
$$

Proof. Consider the endomorphism $\boldsymbol{h} \in \operatorname{End}$ Forget $\left._{\underline{\mathcal{O}}_{c, \nu}}\right)$ acting on Verma objects $M_{c, \nu}(\mu), M_{c, \nu}(\tau)$.

By Proposition 6.2.7, $\boldsymbol{h}_{M_{c, \nu}(\mu)}$ acts on $X_{\mu} \subset M_{c, \nu}(\mu)$ with generalized eigenvalue $h_{c, \nu}(\mu)$, while $\boldsymbol{h}_{M_{c, \nu}(\tau)}$ acts on $\phi\left(X_{\mu}\right) \subset S^{m} \mathfrak{h}_{0}^{*} \otimes X_{\tau}$ with generalized eigenvalue $h_{c, \nu}(\tau)+m$. We conclude that

$$
h_{c, \nu}(\tau)+m=h_{c, \nu}(\mu) .
$$

That is,

$$
\begin{aligned}
& \frac{\nu-1}{2}-c \cdot\left(\frac{(\nu-|\tau|)(\nu-|\tau|-1)}{2}-|\tau|+c t(\tau)\right)+m \\
& =\frac{\nu-1}{2}-c \cdot\left(\frac{(\nu-|\mu|)(\nu-|\mu|-1)}{2}-|\mu|+c t(\mu)\right)
\end{aligned}
$$

which can be rewritten as

$$
c \cdot\left(\frac{|\tau|^{2}-|\mu|^{2}-(|\tau|-|\mu|)}{2}-(|\tau|-|\mu|) \nu+c t(\tau)-c t(\mu)\right)=m .
$$

Remark 8.1.2. Note that if $c=0$, equation (8.1.1) implies that $m=0, \mu=\tau$ and the morphism $M(\mu) \longrightarrow M(\tau)$ is the identity map. This means that for $c=0$, all the Verma objects are simple. The category $\mathcal{O}\left(H_{0}(\nu)\right)$ is a continuation of the categories of modules over $\mathbb{C} S_{n} \ltimes \mathbb{A}_{n}$, which are $\mathcal{O}$-coherent $D$-modules and whose Fourier transform has support $\{0\}$. Here $\mathbb{A}_{n}$ is the $n$-th Weyl algebra (the algebra of differential operators on $\mathbb{C}^{n}$ ).

From now on, we will assume that $c \neq 0$ and denote:

Notation 8.1.3. $c^{\prime}:=\frac{1}{c}$.

Remark 8.1.4. In this notation, equation (8.1.1) can be rewritten as

$$
\frac{|\tau|^{2}-|\mu|^{2}-(|\tau|-|\mu|)}{2}+c t(\tau)-c t(\mu)=m c^{\prime}+(|\tau|-|\mu|) \nu
$$

Notation 8.1.5. Let $\tau, \mu$ be Young diagrams, and let $m$ be a positive integer. Denote by $\mathcal{L}_{\tau, \mu, m}$ the set of points $\left(c^{\prime}, \nu\right)$ in $\mathbb{C}^{2}$ satisfying equation (8.1.2).

The above proposition shows (with the notations as in Notation 1.1.1): $\mathcal{B}_{\tau, \mu} \subset$ $\biguplus_{m \in \mathbb{Z}_{>0}} \mathcal{L}_{\tau, \mu, m}$.

Example 8.1.6. $\mathcal{L}_{\tau, \tau, 0}=\mathbb{C}^{2}$ and $\mathcal{L}_{\tau, \tau, m}=\emptyset$ for any Young diagram $\tau$ and any $m \in \mathbb{Z}_{>0}$. 
Notation 8.1.7. Let $\tau, \mu$ be Young diagrams, and $m$ a positive integer. Denote $\mathcal{B}_{\tau, \mu, m}:=\mathcal{L}_{\tau, \mu, m} \cap \mathcal{B}_{\tau, \mu}$.

Remark 8.1.8. Note that Proposition 8.1.1 implies the following statement:

Fix $c, \nu$, and consider the lowest weight $X_{\mu}$ of the Verma object $M_{c, \nu}(\mu)$. Then for all non-trivial maps $M_{c, \nu}(\mu) \longrightarrow M_{c, \nu}(\tau)$ the degree $m$ of $M_{c, \nu}(\tau)$ in which $X_{\mu}$ sits is the same, since it is given by equation (8.1.2).

As a special case of Proposition 8.1.1 we have the following lemma (for its statement and proof for the classical Cherednik algebra, see [11, Lemma 3.5]):

Lemma 8.1.9. Let $X_{\mu} \subset \mathfrak{h}_{0}^{*} \otimes X_{\tau}$ in $\operatorname{Rep}\left(S_{\nu}\right)$. We can regard $X_{\mu}$ as sitting in degree 1 of the $\underline{\mathcal{O}}_{c, \nu}$-object $M_{c, \nu}(\tau)$. Then there is a morphism $M_{c, \nu}(\mu) \longrightarrow M_{c, \nu}(\tau)$ in $\underline{\mathcal{O}}_{c, \nu}$, inducing $\operatorname{Id}_{X_{\mu}}$ on $X_{\mu}$, if and only if

$$
\frac{|\tau|^{2}-|\mu|^{2}-(|\tau|-|\mu|)}{2}+c t(\tau)-c t(\mu)=c^{\prime}+(|\tau|-|\mu|) \nu
$$

Proof. Consider the morphism $\left.y_{M_{c, \nu}(\tau)}\right|_{\mathfrak{h}_{0}^{*} \otimes X_{\tau}}: \mathfrak{h}_{0} \otimes \mathfrak{h}_{0}^{*} \otimes X_{\tau} \longrightarrow X_{\tau}$. By the commutation relations in Section [5, we have an equality between the following morphisms $\mathfrak{h}_{0} \otimes \mathfrak{h}_{0}^{*} \otimes X_{\tau} \longrightarrow X_{\tau}$ in $\operatorname{Rep}\left(S_{\nu}\right)$ :

$$
\begin{gathered}
\left.y_{M_{c, \nu}(\tau)}\right|_{\mathfrak{h}_{0}^{*} \otimes X_{\tau}} \circ\left(\operatorname{Id}_{\mathfrak{h}_{0}} \otimes x_{M_{c, \nu}(\tau)} \mid X_{\tau}\right)-x_{M_{c, \nu}(\tau)} \circ\left(\operatorname{Id}_{\mathfrak{h}_{0}^{*}} \otimes y_{M_{c, \nu}(\tau)} \mid X_{\tau}\right) \\
\circ\left(c_{\mathfrak{h}_{0}, \mathfrak{h}_{0}^{*}} \otimes \operatorname{Id}_{X_{\tau}}\right)=\left(e v_{\mathfrak{h}_{0}, \mathfrak{h}_{0}^{*}} \otimes X_{\tau}\right) \circ\left(\mathrm{Id}-c \Omega^{3}-c \Omega^{23}\right) .
\end{gathered}
$$

Furthermore, the definition of $x_{M_{c, \nu}(\tau)}, y_{M_{c, \nu}(\tau)}$ tells us that the right-hand side of the above equality is just $\left.y_{M_{c, \nu}(\tau)}\right|_{\mathfrak{h}_{0}^{*} \otimes X_{\tau}}$, so

$$
\left.y_{M_{c, \nu}(\tau)}\right|_{\mathfrak{h}_{0}^{*} \otimes X_{\tau}}=\left(e v_{\mathfrak{h}_{0}, \mathfrak{h}_{0}^{*}} \otimes_{X_{\tau}}\right) \circ\left(\mathrm{Id}-c \Omega^{3}-c \Omega^{23}\right) .
$$

Denote by $Y$ the endomorphism the isomorphism of $\mathfrak{h}_{0}^{*} \otimes X_{\tau}$ corresponding to the morphism $\left.y_{M_{c, \nu}(\tau)}\right|_{\mathfrak{h}_{0}^{*} \otimes X_{\tau}}$ under the correspondence

$$
\operatorname{Hom}_{\operatorname{Rep}\left(S_{\nu}\right)}\left(\mathfrak{h}_{0} \otimes \mathfrak{h}_{0}^{*} \otimes X_{\tau}, X_{\tau}\right) \cong \operatorname{End}_{\operatorname{Rep}\left(S_{\nu}\right)}\left(\mathfrak{h}_{0}^{*} \otimes X_{\tau}\right) .
$$

We immediately see that

$$
Y=\operatorname{Id}-c \Omega^{2}-c \Omega^{12}
$$

(note that in the classical category $\mathcal{O}\left(H_{c}(n)\right)$, we would have: $Y=1-c \sum_{s \in S} 1 \otimes$ $\left.s+c \sum_{s \in S} s \otimes s\right)$.

By definition, an indecomposable object $X_{\mu} \subset \mathfrak{h}_{0}^{*} \otimes X_{\tau}$ is singular in $M_{c, \nu}(\tau)$ iff $\left.y_{M_{c, \nu}(\tau)}\right|_{X_{\mu}}=0$, which is equivalent to requiring that $\left.Y\right|_{X_{\mu}}=0$.

As it was pointed out in Subsection 4.3, the endomorphism $c \Omega^{2}=c \operatorname{Id}_{\mathfrak{h}_{0}^{*}} \otimes \Omega$ has a unique generalized eigenvalue

$$
c \cdot C_{\nu}(\tau)=h_{c, \nu}(\tau)-\frac{\nu-1}{2}
$$

on $\mathfrak{h}_{0}^{*} \otimes X_{\tau}$ (i.e. $c \Omega^{2}-c \cdot C_{\nu}(\tau)$ is a nilpotent endomorphism of $\mathfrak{h}_{0}^{*} \otimes X_{\tau}$ ). Similarly, the restriction of the endomorphism $c \Omega^{12} \in \operatorname{End}_{\operatorname{Rep}\left(S_{\nu}\right)}\left(\mathfrak{h}_{0}^{*} \otimes X_{\tau}\right)$ to $X_{\mu}$ is just $\Omega_{X_{\mu}}$, and has a unique generalized eigenvalue

$$
c \cdot C_{\nu}(\mu)=h_{c, \nu}(\mu)-\frac{\nu-1}{2} .
$$

Thus $Y$ acts on an indecomposable object $X_{\mu} \subset \mathfrak{h}_{0}^{*} \otimes X_{\tau}$ with the generalized eigenvalue

$$
1+h_{c, \nu}(\tau)-\frac{\nu-1}{2}-\left(h_{c, \nu}(\mu)-\frac{\nu-1}{2}\right)=1+h_{c, \nu}(\tau)-h_{c, \nu}(\mu) .
$$



iff

This proves that $X_{\mu}$ is an indecomposable singular $\underline{\operatorname{Rep}}\left(S_{\nu}\right)$-subobject of $M(\tau)$

$1+h_{c, \nu}(\tau)-h_{c, \nu}(\mu)=0$, which is equivalent to the requirement

$$
\frac{|\tau|^{2}-|\mu|^{2}-(|\tau|-|\mu|)}{2}+c t(\tau)-c t(\mu)=c^{\prime}+(|\tau|-|\mu|) \nu .
$$

An additional condition for $X_{\mu}$ to sit in degree $m$ of $M_{c, \nu}(\tau)$ arises from Pieri's rule (cf. Proposition 4.2.1).

In terms of Notations 8.1.5 8.1.7 Lemma 8.1.9 means that $\mathcal{B}_{\tau, \mu, 1}=\mathcal{L}_{\tau, \mu, 1}$ whenever $\mu$ is obtained from $\tau$ as in Pieri's rule, i.e. by adding/moving/deleting a cell, or if $\mu=\tau$.

8.2. Remarks on equation (8.1.2). For a Young diagram $\mu$, denote:

$$
f(\mu):=\frac{|\mu|^{2}-|\mu|}{2}+\operatorname{ct}(\mu)
$$

Then equation (8.1.2) can be rewritten as

$$
f(\tau)-f(\mu)=c^{\prime} m+(|\tau|-|\mu|) \nu .
$$

Lemma 8.2.1. Let $\mu$ be a Young diagram. Then $f(\mu)$ is a non-negative integer less or equal to $|\mu|^{2}-|\mu|$, and it is equal to zero if $\mu$ is a column diagram (i.e. $l(\mu) \leq 1)$, and to $|\mu|^{2}-|\mu|$ if $\mu$ is a row diagram (i.e. $l(\mu) \leq 1$ ).

Proof. This is equivalent to saying that $|c t(\mu)| \leq \frac{|\mu|^{2}-|\mu|}{2}$. The latter can be proved by induction on the number of columns of $\mu$ :

Base: Assume the number of columns of $\mu$ is zero, i.e. $\mu=\emptyset$. Then $|c t(\mu)|=$ $0=\frac{|\mu|^{2}-|\mu|}{2}$. Also, if $\mu$ is a column diagram, then $\operatorname{ct}(\mu)=-\frac{|\mu|^{2}-|\mu|}{2}$.

Step: Denote by $k$ the number of columns of $\mu(k>1)$, by $\mu^{\prime}$ the diagram $\mu$ without the last column, and by $l$ the number of boxes in the last column of $\mu$. By induction assumption, $\left|\operatorname{ct}\left(\mu^{\prime}\right)\right| \leq \frac{\left|\mu^{\prime}\right|^{2}-\left|\mu^{\prime}\right|}{2}$. Next, $\operatorname{ct}(\mu)=\operatorname{ct}\left(\mu^{\prime}\right)+$ $(k-1) \cdot l-\ell(l-1) / 2$, and so $\frac{|\mu|^{2}-|\mu|}{2}=\frac{\left|\mu^{\prime}\right|^{2}-\left|\mu^{\prime}\right|}{2}+\left|\mu^{\prime}\right| l+\frac{l^{2}-l}{2} \geq\left|c t\left(\mu^{\prime}\right)\right|+\frac{l^{2}-l}{2}+\left|\mu^{\prime}\right| l \geq$ $\left|c t\left(\mu^{\prime}\right)+(k-1) \cdot l-\ell(l-1) / 2\right|=|c t(\mu)|^{2}$ (for the last inequality, note that by definition, $\left|\mu^{\prime}\right| \geq k-1$, with equality if and only if $\mu$ is a row diagram). For a row diagram $\mu$ (with $k$ cells), there is an equality $\operatorname{ct}(\mu)=\frac{|\mu|^{2}-|\mu|}{2}$, and in general, $|c t(\mu)|<\frac{|\mu|^{2}-|\mu|}{2}$ for $\mu$ having $k>1$ columns and not a row diagram.

For the right-hand side of equation (8.2.1), note that we have, by Pieri's rule (Proposition 4.2.1): $m \geq|| \mu|-| \tau||$.

\section{Blocks of the Category $\mathcal{O}\left(H_{c}(n)\right)$ (Classical Case)}

9.1. KZ functor and connection to the representations of the Hecke algebras. A powerful tool in studying the category $\mathcal{O}$ for the Cherednik algebra is the KZ functor (see e.g. [15, 2.8.2], [10, Section 6], [3, Section 3.3]). The KZ functor is a functor

$$
\mathcal{O}\left(H_{c}(n)\right) \longrightarrow \operatorname{Rep}\left(\mathcal{H}_{q}(n)\right) \text {. }
$$

$\operatorname{Rep}\left(\mathcal{H}_{q}(n)\right)$ being the category of representations of the Hecke algebra $\mathcal{H}_{q}(n)$ of type A, where $q=\exp (2 \pi i c)$. 
It turns out that this functor is essentially surjective on objects, surjective on Homs and exact (see [13]). This functor induces an equivalence of categories $\overline{K Z}$ : $\mathcal{O} / \mathcal{O}^{\text {tor }} \longrightarrow \operatorname{Rep}\left(\mathcal{H}_{q}(n)\right)$, where $\mathcal{O}^{\text {tor }}$ is the full subcategory of the category $\mathcal{O}$ whose objects are modules which, when considered as $\mathbb{C}\left[\mathfrak{h}_{0}\right]$-modules, have Krull dimension less than $n-1$ (see [10, Section 6.3]). The non-zero objects of $\mathcal{O} / \mathcal{O}^{\text {tor }}$ would thus be $H_{c}(n)$-modules which are supported on the whole $\mathbb{C}^{n}$.

Moreover, by [2, Proposition 2.10] (also proved in [13]), the KZ functor is faithful on the full subcategory of Verma modules in $\mathcal{O}\left(H_{c}(n)\right)$.

We will use the fact that the $K Z$ functor takes $M(\lambda)$ to $S_{\lambda}$ (the dual of the Specht module) if $c \geq 0$, and to $S_{\lambda}$ (Specht module) if $c<0$. For $c<0$, the module $L(\lambda)$ goes to $D_{\lambda}$ (which is either the unique simple quotient of the Specht module $S_{\lambda}$, if it exists, or zero).

9.2. Representations of Hecke algebras of type A. We will use the following facts about the representations of Hecke algebras of type A (see [19], 3, Section $3])$ :

Some definitions:

Notation 9.2.1. Denote $e:=\operatorname{ord}_{\mathbb{C}}(q)$ (i.e. $e$ is the order of $q$ if $q$ is a root of unity and $\infty$ otherwise), and denote $s:=n-e$.

First, we have:

Theorem 9.2.2. If $e>n$ (in particular, if $e=\infty$ ), then the category $\operatorname{Rep}\left(\mathcal{H}_{q}(n)\right)$ is semisimple.

From now on, we will assume $e \leq n$, and thus $0 \leq s \leq n$.

Definition 9.2.3 (e-hook).

- Hooks are parameterized by cells $(i, j)$ in the diagram; a hook corresponding to $(i, j)$ consists of all the cells $\left(i^{\prime}, j^{\prime}\right)$ such that either $i^{\prime}=i, j^{\prime} \geq j$, or $i^{\prime} \geq i, j^{\prime}=j$. The cell $(i, j)$ is the "vertex" of the hook corresponding to it. Cells $\left(i, j^{\prime}\right), j^{\prime}>j$ are called the "arm" of the hook, while cells $\left(i^{\prime}, j\right), i^{\prime}>i$ are called the "leg" of the hook.

- An $e$-hook of a Young diagram is a hook of length $e$ (i.e. contains exactly $e$ cells).

Example 9.2.4. Let $\lambda=(5,4,2,2)$. The hook corresponding to $(1,2)$ is a 7 -hook with arm 3 and leg 3 :

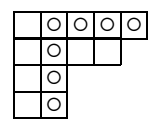

Lemma 9.2.5. For $n$ large relatively to $s$ (namely, $n>2 s, n>1$ ), a Young diagram $\tau$ with $|\tau|=n$ can have at most one e-hook.

Proof. Given two distinct hooks of the same length, their intersection can contain at most one cell. Now, fix a Young diagram $\tau$ with $|\tau|=n$. If $\tau$ has two distinct $e$-hooks, we obtain the inequality $2 e-1 \leq|\tau|=n$, and thus $n \leq 2 s+1$. Note that, in fact, a Young diagram cannot consist of two hooks of equal size intersecting each other in one cell unless this diagram contains just one cell. So if $n \geq 2$, then $2 e-1<|\tau|=n$, i.e. $n<2 s+1$. So for $n>2 s, n>1$, there is at most one $e$-hook in $\tau$. 
Definition 9.2.6 (Core). Let $s \leq n$ be a non-negative integer. The $(n-s)$-core of a Young diagram $\tau$ is the Young diagram obtained by performing the following procedure on $\lambda:=\tau$ :

(1) Take the Young diagram $\lambda$.

(2) If $\lambda$ has no $(n-s)$-hook, stop. The diagram $\lambda$ is then the $(n-s)$-core of $\tau$.

(3) Otherwise, remove an $(n-s)$-hook from $\lambda$ and move the boxes underneath this $(n-s)$-hook one position up and one position to the left. Denote the obtained Young diagram by $\lambda^{(1)}$.

(4) Repeat the procedure for $\lambda:=\lambda^{(1)}$

So for $n>\max (2 s, 1)$, either $\operatorname{core}_{(n-s)}(\tau)=\tau$, or $\operatorname{core}_{(n-s)}(\tau)$ has $s$ boxes.

Example 9.2.7. Let $\lambda=(5,4,2,2)$. Its 7 -core is obtained by removing the hook corresponding to $(1,2)$ and moving the boxes below it one space up and left, i.e. $\operatorname{core}_{7}(\lambda)=(3,1,1,1)$ :

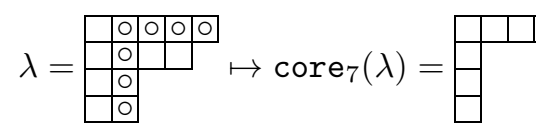

Now, assume $n>\max (2 s, 1)$. Classification of partitions of size $n$ with given core $\lambda$ such that $|\lambda|=s$ is given by the following proposition:

Proposition 9.2.8. Let $\lambda$ be a Young diagram of size s. Let hook $(l,(n-s))$ $(0 \leq l \leq(n-s-1))$ be the $(n-s)$-hook with leg $l:((n-s)-l, 1,1, \ldots, 1)$. Then there is a unique Young diagram $\operatorname{rec}(l, \lambda)$ of size $n$ such that $\lambda$ is obtained by deleting the hook hook $(l, n-s)$ from $\operatorname{rec}(l, \lambda)$ (thus $\lambda$ is the $(n-s)$-core of $\operatorname{rec}(l, \lambda))$.

Proof. Basically, $\operatorname{rec}(l, \lambda)$ is obtained by "inserting" a $h o o k(l,(n-s))$ to $\lambda$ in a certain not completely trivial way. To insert a hook hook $(l,(n-s))$ into a diagram $\lambda$, one should put its vertex in the position $(i, j)$ such that the following equations are satisfied:

$$
\begin{aligned}
& \lambda_{i}+1 \leq j-1+n-s-l \leq \lambda_{i-1}, \\
& \lambda_{j}+1 \leq i+l \leq \lambda_{j-1}
\end{aligned}
$$

$\left(\lambda_{0}, \lambda_{0}:=\infty\right)$.

We now explain how to find such $i, j$.

First, we show that if the inequalities (9.2.1) hold, then $i=1$ or $j=1$. Indeed, adding the two inequalities (9.2.1), we obtain:

$$
j-1+n-s-l+i+l=i+j-1+n-s \leq \lambda_{i-1}+\lambda_{j-1} .
$$

But $n-s>s=|\lambda|$, so $i+j-1+n-s \geq i+j+|\lambda| \geq|\lambda|+2$. Thus we obtain: $|\lambda|+2 \leq \lambda_{i-1}+\lambda_{j-1}$. But this is clearly possible only when $i=1$ or $j=1$.

Next, find $j$ such that

$$
\lambda_{j}+1 \leq l+1 \leq \lambda_{j-1}
$$

(which is always possible). We now have two cases:

(1) If we obtain $j \geq 2$, then we put $i:=1$ and we only need to check that $\lambda_{1}+1 \leq j-1+n-s-l$. But we assumed that $n>2 s$, so $n-s>|\lambda|=s$, and thus

$$
j-1+n-s-l \geq j+|\lambda|-l \geq 2+|\lambda|-\lambda_{j-1} \geq \lambda_{1}+1
$$

so we found indices $(i, j)$ satisfying the inequalities (9.2.1). 
(2) If we obtain $j=1$, then $\lambda_{j} \leq l$. We now find $i$ such that

$$
\lambda_{i}+1 \leq n-s-l \leq \lambda_{i-1}
$$

(again, this is always possible). To show that we found indices $(i, j)$ satisfying the inequalities (9.2.1), we only need to check that $\lambda_{1}+1 \leq i+l$, which is true since we have $i \geq 1$ and $\lambda_{j} \leq l$.

This shows that there exists exactly one way to insert the hook hook $(l,(n-s))$ into $\lambda$.

Example 9.2.9. Let $\lambda=(5,4,2,2)$.

(1) Adding the 7 -hook $(6,1)$ to its 7 -core $\left(\operatorname{core}_{7}(\lambda)=(3,1,1,1)\right)$, we obtain the Young diagram $\operatorname{rec}\left(1, \operatorname{core}_{7}(\lambda)\right)=(7,4,1,1)$ :

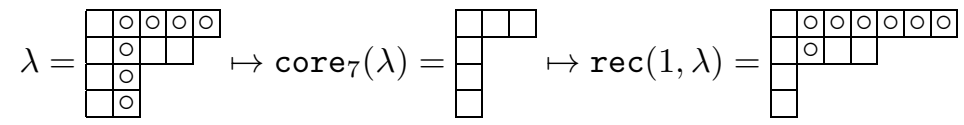

(2) Adding the 7 -hook $(2,1,1,1,1,1)$ to $\operatorname{core}_{7}(\lambda)$, we obtain the Young diagram $\operatorname{rec}\left(5, \operatorname{core}_{7}(\lambda)\right)=(3,2,2,2,2,1,1)$ :

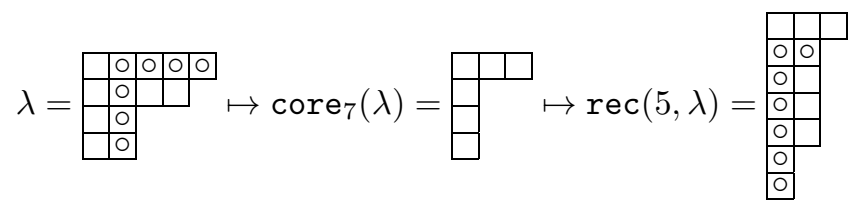

Definition 9.2.10. Let $e \in \mathbb{Z}_{+}$.

- A partition $\lambda$ is called $e$-regular if the multiplicity of each part of $\lambda$ is smaller than $e$.

- A partition $\lambda$ is called $e$-restricted if $\lambda_{i}-\lambda_{i+1}<e$ for all $i$ (i.e. if $\lambda^{2}$ is $e$-regular).

Theorem 9.2.11 (cf. [19]). Let $\tau, \mu$ be partitions of $n$.

(1)

- All the simple modules of $\mathcal{H}_{q}(n)$ are $D_{\lambda}$, where $\lambda$ is e-regular. These are exactly the partitions for which $D_{\lambda} \neq 0$.

- The $\mathcal{H}_{q}(n)$-modules $S_{\tau}{ }^{\sim}$ and $S_{\tau}$ have the same composition factors.

- Two modules $S_{\tau}, S_{\mu}$ belong to the same block iff $\tau, \mu$ have the same $(n-s)$-core.

- If $\tau$ has no $(n-s)$-hook, then $S_{\tau}$ (and hence $S_{\tau}{ }^{2}$ ) is irreducible.

(2) Assume $n>2 s$. Then we have:

- Let $\tau=\operatorname{rec}(l, \beta), 0 \leq l \leq(n-s-2)$ and $\mu \neq \tau$. Then there is a non-trivial morphism $S_{\mu} \rightarrow S_{\tau}$ iff $\mu=\operatorname{rec}(l+1, \beta)$.

- If $\tau=\operatorname{rec}(l, \beta), \mu=\operatorname{rec}(l+1, \beta)$, then the composition factors of $S_{\tau}$ are $D_{\tau}, D_{\mu}$, with multiplicity 1 (only one of them if the other is zero).

9.3. Blocks of the category $\mathcal{O}\left(H_{c}(n)\right)$. We now give the relevant results for the category $\mathcal{O}\left(H_{c}(n)\right)$ :

Let $n$ be a non-negative integer.

9.3.1. Equivalences. First, we have a useful theorem proved by Rouquier and expanded by Losev (see [21, Theorem 5.12], 17]): 
Theorem 9.3.1 (Rouquier). Let $n>1, r, a>0, b \neq 0$ be integers, $\operatorname{gcd}(r, a)=1$. Then the categories $\mathcal{O}\left(H_{c_{1}=b / a}(n)\right), \mathcal{O}\left(H_{c_{2}=(b r) / a}(n)\right)$ are equivalent if $c_{1} \notin\left\{\frac{2 k+1}{2}, k \in \mathbb{Z}\right\}$, with a correspondence: morphism $M_{c_{1}, n}(\mu) \longrightarrow M_{c_{1}, n}(\tau)$ $(|\tau|=|\mu|=n)$ such that $\mu$ sits in degree $m$ of $M_{c_{1}, n}(\tau)$ corresponds to morphism $M_{c_{2}, n}(\mu) \longrightarrow M_{c_{2}, n}(\tau)(|\tau|=|\mu|=n)$ such that $\mu$ sits in degree $r m$ of $M_{c_{2}, n}(\tau)$.

Next, we have the following simple equivalence of categories (see [22, 3.1.4]):

Observation 9.3.2. The rational Cherednik algebras $H_{-c}(n), H_{c}(n)$ are isomorphic:

$$
H_{c}(n) \longrightarrow H_{(-c)}(n), x \longmapsto x, y \longmapsto y, \sigma \in S_{n} \longmapsto(\operatorname{sign}(\sigma) \cdot \sigma) \in \mathbb{C}\left[S_{n}\right] .
$$

This means that the categories of representations of these algebras are equivalent, with equivalence given by

$$
\mathcal{O}\left(H_{c}(n)\right) \longrightarrow \mathcal{O}\left(H_{(-c)}(n)\right), V \longmapsto \operatorname{sign} \otimes V,
$$

where sign is the sign representation of $S_{n}$. Note that $\operatorname{sign} \otimes \mu \cong \mu$ for representation $\mu$ of $S_{n}$.

We also have the following statement (see [13, Section 6.2]):

Proposition 9.3.3. If $c \notin \frac{1}{2}+\mathbb{Z}$, then

$$
\operatorname{Hom}_{H_{c}(n)}\left(M_{c, n}(\mu), M_{c, n}(\tau)\right) \cong \operatorname{Hom}_{H_{(-c)}(n)}\left(M_{(-c), n}(\tau), M_{(-c), n}(\mu)\right) .
$$

9.3.2. Results on $\mathcal{O}\left(H_{c}(n)\right)$. The correspondence between the lowest weight representations of $H_{c}(n)$ and the finite-dimensional representations of the Hecke algebra $\mathcal{H}_{q}(n)$ gives us the following theory (see [8]):

First, we have (see [10, 6.13]):

Proposition 9.3.4. For $c \leq 0, K Z\left(M_{c, n}(\tau)\right)=S_{\tau}, K Z\left(L_{c, n}(\tau)\right)=D_{\tau}$, and thus $K Z\left(L_{c, n}(\tau)\right) \neq 0$ iff $\tau$ is $(n-s)$-regular.

For $c>0, K Z\left(L_{c, n}(\tau)\right) \neq 0$ iff $\tau$ is $(n-s)$-restricted.

We next have the following theorem:

Theorem 9.3.5 (Dipper, James; cf. [7]). If $c^{\prime}$ satisfies one of the following conditions:

- $c^{\prime} \notin \mathbb{Q}$,

- $c^{\prime} \in \mathbb{Q}$, and $c^{\prime}=\frac{d}{a}, \operatorname{gcd}(a, d)=1, d>n$,

then the category $\mathcal{O}\left(H_{c}(n)\right)$ is semisimple (in particular, all Verma modules are simple).

Proof. This is a direct consequence of Theorem 9.2.2, the standard property of Verma modules (Proposition 6.2.8) and the fact that the KZ functor is faithful on the full subcategory of Verma modules in $\mathcal{O}\left(H_{c}(n)\right)$.

This means that we remain with only one interesting case:

$$
c^{\prime}=\frac{d}{a}, \operatorname{gcd}(a, d)=1, a>0,0 \leq d \leq n .
$$

For $d \leq n / 2$, the theory is more complicated, but for $d>n / 2$, it is rather simple and explained below. Note that Theorem 9.3.1 allows us to assume that $a=1$ and study just the case $c^{\prime}=d \in\{0,1, \ldots, n\}$.

Fix integer $s \geq 0$, and from now on, consider $n>2 s, c^{\prime}=d=n-s$, and a Young diagram $\tau$ with $|\tau|=n$. 
Corollary 9.3.6. $M_{c, n}(\tau)$ is simple if and only if $\tau$ has no $(n-s)$-hook, or its $(n-s)$-hook is a vertical strip.

Proof. This is a consequence of Theorem 9.2.11 and of the standard property of Verma modules (Proposition 6.2.8).

Theorem 9.3.7. Let $s \geq 0, n>2 s$ be integers, and put $c^{\prime}:=n-s$. Let $\beta$ be an arbitrary Young diagram of size s. Let $\tau=\operatorname{rec}(l, \beta)$, with $0 \leq l \leq(n-s-2)$ and $\mu \neq \tau$ be a Young diagram of size $n$. $1, \beta)$.

Then there is a non-trivial morphism $\psi_{l}: M_{c, n}(\mu) \rightarrow M_{c, n}(\tau)$ iff $\mu=\operatorname{rec}(l+$

In that case, we have:

- $\operatorname{dim} \operatorname{Hom}\left(M_{c, n}(\mu), M_{c, n}(\tau)\right)=1$.

- There is a short exact sequence

$$
0 \longrightarrow L_{c, n}(\mu) \longrightarrow M_{c, n}(\tau) \longrightarrow L_{c, n}(\tau) \longrightarrow 0 .
$$

Proof. This is a generalization of the result in [3, Section 3] (there it is proved for the case $s=0$, i.e. $e=n$ ).

First, recall from [2, Proposition 2.10] (also proved in [13]) that the KZ functor is fully faithful on the full subcategory of Verma modules in $\mathcal{O}\left(H_{c}(n)\right)$. Then Theorem 9.2.11 implies that the dimension of $\operatorname{Hom}\left(M_{c, n}(\mu), M_{c, n}(\tau)\right)$ is one iff $\mu=\operatorname{rec}(l+1, \beta)$, and zero otherwise.

To prove the rest of the theorem, we only need to check that $L_{c, n}(\mu)$ appears among the composition factors of $M_{c, n}(\tau)$ with multiplicity at most one if $\mu=$ $\operatorname{rec}(l+1, \beta)$, and with multiplicity zero otherwise.

If $K Z\left(L_{c, n}(\mu)\right) \neq 0$, then the statement follows immediately from Theorem $9.2 .11(2)$. So we only need to show that the same is true when $K Z\left(L_{c, n}(\mu)\right)=0$. Recall from Proposition 9.3.4 that the latter happens iff $\mu$ is not $(n-s)$-restricted, so we will assume that this is the case.

We now have two possible cases:

- The Young diagram $\mu$ does not have an $(n-s)$-hook, and thus core ${ }_{(n-s)}(\mu)$ $=\mu$. In this case $M_{c, n}(\mu)$ is simple and lies in a semisimple block (see Corollary 9.3.6), so $L_{c, n}(\mu)$ cannot appear as a composition factor in $M_{c, n}(\tau)$ unless $\mu=\tau$ (which is clearly impossible, since $\operatorname{core}_{(n-s)}(\tau)=\beta$, which has size $s<n)$.

- The Young diagram $\mu$ has an $(n-s)$-hook. Denote: $\beta^{\prime}:=\operatorname{core}_{(n-s)}(\mu)$. It is then easy to see that the condition that $\mu$ is not $(n-s)$-restricted implies that $\mu$ is obtained from $\beta^{\prime}$ by adding $(n-s)$ cells to the first row (i.e. $\left.\mu=\operatorname{rec}\left(0, \beta^{\prime}\right)\right)$.

Assume $M_{c, n}(\mu), M_{c, n}(\tau)$ lie in the same block of $\mathcal{O}\left(H_{c}(n)\right)$ (otherwise we are done). Since the KZ functor is fully faithful on the full subcategory of Verma modules in $\mathcal{O}\left(H_{c}(n)\right)$, the modules $S_{\mu} \cong K Z\left(M_{c, n}(\mu)\right), S_{\tau}{ }^{\sim} \cong$ $K Z\left(M_{c, n}(\tau)\right)$ belong to the same block of $\operatorname{Rep}\left(\mathcal{H}_{q}(n)\right)$ (here $\left.q=\exp (2 \pi i c)\right)$. By Theorem 9.2.11(1), this implies that $\beta=\beta^{\prime}$.

So it remains to check that for $l>0$ and $\mu=\operatorname{rec}(0, \beta), L_{c, n}(\mu)$ does not appear among the composition factors of $M_{c, n}(\tau)$. Indeed, since $\mu=$ $\operatorname{rec}(0, \beta), \tau=\operatorname{rec}(l, \beta)$ and $l>0$, we have: $c t(\tau)<c t(\mu)$, and thus

$$
h_{c, n}(\tau)=\frac{n-1}{2}-c \cdot c t(\tau)>\frac{n-1}{2}-c \cdot c t(\mu)=h_{c, n}(\mu) .
$$


But $h_{c, n}(\tau)$ is the lowest eigenvalue of $\boldsymbol{h}$ on $M_{c, n}(\tau)$, so $L_{c, n}(\mu)$ cannot be a composition factor of $M_{c, n}(\tau)$.

Example 9.3.8. The Verma module $M_{c, n}\left(\tau^{n-1}\right.$ ) (whose lowest weight is the trivial representation $\tau^{n-1}=\widetilde{\emptyset}(n)$ of $\left.S_{n}\right)$ is reducible if and only if the following condition on $c^{\prime}=\frac{1}{c}, n$ holds:

$$
c^{\prime}=\frac{n-s}{r}, s \geq 0, r \geq 1 .
$$

In this case, $M_{c, n}\left(\tau^{n-1}\right)$ contains a lowest weight $H_{c}(n)$-submodule, whose lowest weight $\mu$ lies in degree $r(s+1)$ of $M_{c, n}\left(\tau^{n-1}\right)$. The Young diagram of $\mu$ is a two row diagram, the lower row containing $s+1$ boxes.

Theorem 9.3.7 gives the following corollary:

Corollary 9.3.9. Let $n>3, s \geq 0, r \geq 1$ be integers, $n>2 s, \operatorname{gcd}(r, n-s)=1$. Then the blocks of the category $\mathcal{O}\left(H_{c=\frac{r}{n-s}}(n)\right)$ correspond to $(n-s)$-cores, and we have two types of blocks: blocks containing only one Verma module (up to isomorphism), corresponding to a diagram which doesn't have an $(n-s)$-hook, and blocks which correspond to Young diagrams of size $s$; in such a block Block $k_{\beta}(|\beta|=s)$ lie the Verma modules whose $(n-s)$-core is $\beta$, and these Verma modules form a long exact sequence:

$$
\begin{aligned}
& 0 \longrightarrow M_{c, n}(\operatorname{rec}((n-s-1), \beta)) \stackrel{\psi_{(n-s-2)}}{\longrightarrow} M_{c, n}(\operatorname{rec}((n-s-2), \beta)) \stackrel{\psi_{(n-s-3)}}{\longrightarrow} \cdots \\
& \cdots \stackrel{\psi_{1}}{\longrightarrow} M_{c, n}(\operatorname{rec}(1, \beta)) \stackrel{\psi_{0}}{\longrightarrow} M_{c, n}(\operatorname{rec}(0, \beta)) \longrightarrow L_{c, n}(\operatorname{rec}(0, \beta)) \longrightarrow 0 .
\end{aligned}
$$

(Note that $\operatorname{rec}((n-s-1), \beta)$ is the diagram $\beta$ with a vertical $(n-s)$-hook added, and $\operatorname{rec}(0, \beta)$ is the diagram $\beta$ with a horizontal $(n-s)$-hook added).

Remark 9.3.10. For $s \geq n / 2$, the situation is more complicated, but one can still say the following (see [8]).

Let $c^{\prime}=n-s$. If $M_{c, n}(\mu), M_{c, n}(\tau)$ belong to the same block, then they have the same $(n-s)$-core. In particular, if $\tau$ has no $(n-s)$-hooks, then $M_{c, n}(\tau)$ is a simple module lying in a semisimple block.

\section{Constructions For $\underline{\mathcal{O}} c, \nu$}

We now define constructions analogous to those described in Section 9 for the category $\underline{\mathcal{O}}_{c, \nu}$, where we consider generic values of $\nu$. We use the idea described in Section 4 of treating a simple object $X_{\tau}$ of $\operatorname{Rep}\left(S_{\nu}\right)$ as a Young diagram obtained by adding a very long top row to $\tau$ ("the top row having length $(\nu-|\tau|)$ )"). This idea is only meant to provide some intuition, but it is true that for $n \in \mathbb{Z}, n>>0$, the image of $X_{\tau}$ under the functor $\operatorname{Rep}\left(S_{\nu=n}\right) \longrightarrow \operatorname{Rep}\left(S_{n}\right)$ is isomorphic to $\tilde{\tau}(n)$, which is exactly a Young diagram obtained by adding a top row of length $(n-|\tau|)$ to $\tau$.

So instead of adding and removing $(n-s)$-hooks as we did in Section 9, we would be adding and removing " $(\nu-s)$-hooks" from diagrams with "very long top row of size $(\nu-|\tau|)$ ". Below is a formal description of the relevant constructions.

Let $\tau$ be a Young diagram, and $s \geq 0$ an integer.

Definition 10.0.11.

- Define $C_{\tau}:=\left\{|\tau|-1+j-\check{\tau}{ }^{\check{1}} \mid j \geq 1\right\}$. 
- For $s \in C_{\tau}, s=|\tau|-1+j_{s}-\widetilde{\tau_{j}}$ for some $j_{s} \geq 1$, define the $(\nu-s)$-core

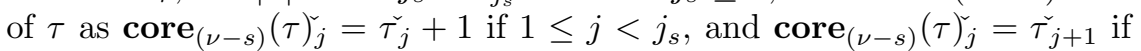
$j \geq j_{s}$.

That is, $\operatorname{core}_{(\nu-s)}(\tau)$ is the Young diagram obtained by taking out column $j_{s}$ of $\tau$, moving columns $1, \ldots, j_{s}-1$ down and adding a row of length $j_{s}-1$ on top of them, and moving left the columns $j_{s}+1, \ldots$

Example 10.0.12. Let $\tau=(8,5,4,3,3,2)$. Then $|\tau|=25, C_{\tau}=\{19,20,22,25,27$, $29,30,31\} \cup \mathbb{Z}_{\geq 33}$. Let $s=22$. Then $s \in C_{\tau}, s=|\tau|-1+3-\check{\tau_{3}}$. So $j_{s}=3$, and $\operatorname{core}_{(\nu-s)}(\tau)=(7,4,3,2,2,2,2)$.

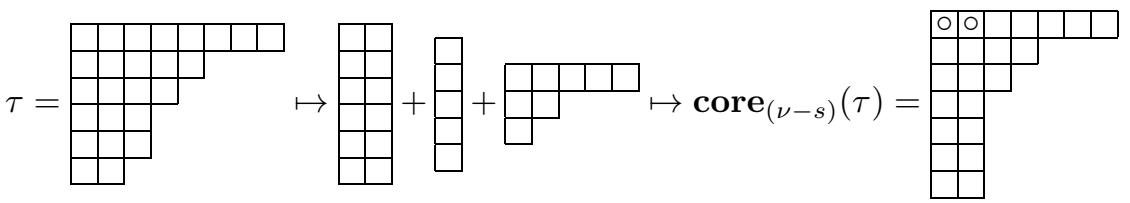

Let $s=34$. Then $s \in C_{\tau}, s=|\tau|-1+10-\check{\tau_{10}}$. So $j_{s}=10$, $\operatorname{core}_{(\nu-s)}(\tau)=$ $(9,8,5,4,3,3,2)$.

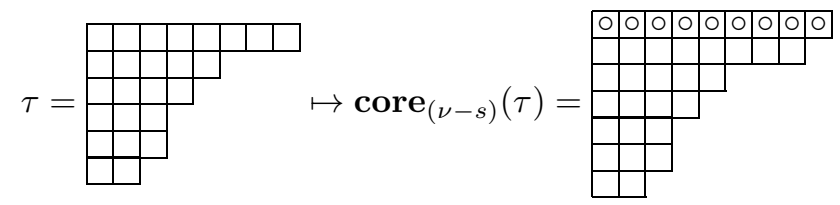

Remark 10.0.13. Deligne defined $\operatorname{core}_{(\nu-s)}(\lambda)$, which he denoted by $\{\lambda\}_{n}^{+}$, with $n:=s$, in [6, 7.5].

The process of "reconstruction" (corresponding to inserting a hook into a Young diagram) is defined as follows:

Construction 10.0.14. Given any Young diagram $\eta$ and an integer $l \geq 0$, we define $\tau:=\operatorname{rec}(l, \eta)$ as the Young diagram obtained through the following steps:

- Find the index $k \geq 1$ such that $\eta_{k-1} \geq l+1>\eta_{k} \check{ }\left(\right.$ here $\eta_{0}:=\infty$ ).

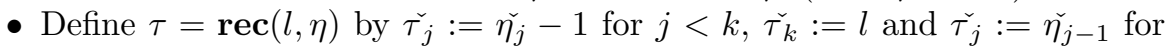
$j>k$.

That is, we divide $\eta$ into two parts: part 1 consisting of columns $1, \ldots, k-1$ and part 2 consisting of columns $k, k+1, \ldots$ Then we delete the top row of part 1 , add a $k$-th column of length $l$, and add part 2 as columns $k+1, k+2, \ldots$.

\section{Example 10.0.15.}

- For $l>\check{\eta_{1}}, \operatorname{rec}(l, \eta)$ is the Young diagram obtained from $\eta$ by adding a column of length $l$ to $\eta$ (this will become the first column).

- For $l=0, \operatorname{rec}(l, \eta)$ is the Young diagram obtained from $\eta$ by removing its top row.

Example 10.0.16. $\operatorname{rec}\left(\tau_{j_{s}}, \operatorname{core}_{(\nu-s)}(\tau)\right)=\tau$.

Example 10.0.17. Let $\tau=(10,8,8,6,5,4,1), s=43$ (so $\left.j_{s}=6\right)$. Then

$$
\operatorname{rec}\left(7, \operatorname{core}_{(\nu-s)}(\tau)\right)=(10,8,8,6,6,6,5) .
$$




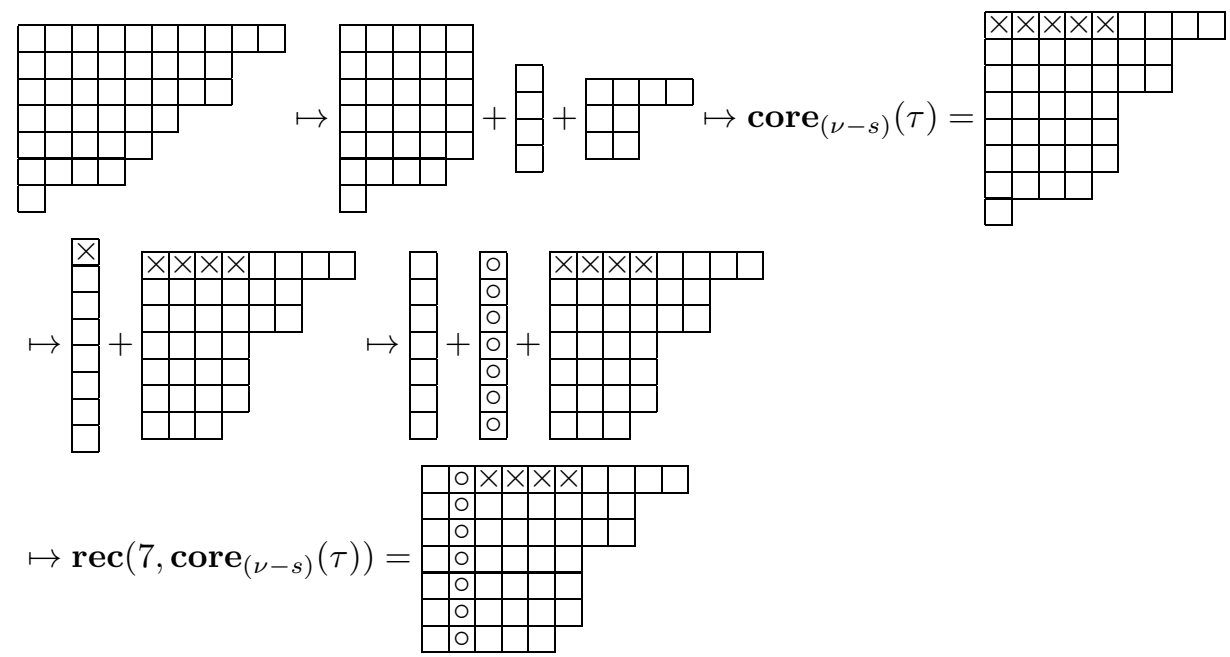

Notation 10.0.18. Let $\tau$ be a Young diagram, $s \in C_{\tau}, l \in \mathbb{Z}_{\geq-\tau \breve{j_{s}}}$ ( $j_{s}$ given by $\left.s=|\tau|-1+j_{s}-\widetilde{\tau_{j_{s}}}\right)$. We will denote the Young diagram $\operatorname{rec}\left(\check{\tau_{j_{s}}}+l, \operatorname{core}_{(\nu-s)}(\tau)\right)$ by $\Gamma(\tau, s, l)$.

Example 10.0.19. Consider $\tau=\emptyset$. Given $s \geq 0$, the $(\nu-s)$-core of $\emptyset$ is a row of length $s$. Then $\Gamma(\emptyset, s, l)=\operatorname{rec}\left(l, \operatorname{core}_{(\nu-s)}(\emptyset)\right)$ is a hook with arm length $s$ and leg length $l-1$.

Lemma 10.0.20. Let $\tau$ be a Young diagram, $l>0$ an integer, $s:=|\tau|-1+j_{s}-\tau_{j_{s}}$ for some $j_{s} \geq 1, \mu=\Gamma(\tau, s, l)$. Then $|\mu|-|\tau|>0$, and $s=\frac{f(\mu)-f(\tau)}{|\mu|-|\tau|}$ ( $f$ is defined in Subsection 8.2).

Proof. We first describe $\mu$ in terms of $\tau$. By Constructions 10.0.11, 10.0.14 described above, $\mu$ is obtained from $\tau$ by taking out column $j_{s}$ of $\tau$, inserting a column of length $\tau_{j_{s}}+l$ after column $k_{s, l}-1$ for some $k_{s, l} \leq j_{s}$ ( $k_{s, l}$ is uniquely determined by Construction 10.0.14), and adding a cell to each of the columns $k_{s, l}+1, \ldots, j_{s}$ of the newly constructed diagram. Thus $\check{\mu_{j}}=\check{\tau_{j}}$ for $j<\check{k_{s, l}}$ and $j>\check{j_{s}}, \check{\mu_{k_{s, l}}}=\check{\tau_{j_{s}}}+l$, and $\check{\mu_{j}}=\check{\tau_{j}}+1$ for $j=k_{s, l}+1, \ldots, j_{s}$. This means that we have:

$$
|\mu|-|\tau|=j_{s}-k_{s, l}+l .
$$

Note that since $k_{s, l} \leq j_{s}, l>0$, we have: $|\mu|-|\tau|>0$.

From this description of $\mu$, we see that

$$
\begin{aligned}
& c t(\mu)-c t(\tau)=-\left(j_{s} \check{\tau_{j_{s}}}-\frac{\check{\tau_{j_{s}}}\left(\check{\tau_{j_{s}}}+1\right)}{2}\right)+\left(\frac{j_{s}\left(j_{s}-1\right)}{2}-\frac{k_{s, l}\left(k_{s, l}-1\right)}{2}\right) \\
& +\left(k_{s, l}\left(\check{\tau_{j_{s}}}+l\right)-\frac{\left(\check{\tau_{j_{s}}}+l\right)\left(\check{\tau_{j_{s}}}+l+1\right)}{2}\right) \\
& =-\widetilde{\tau_{j_{s}}}\left(j_{s}-k_{s, l}+l\right)+\frac{j_{s}\left(j_{s}-1\right)}{2}-\frac{k_{s, l}\left(k_{s, l}-1\right)}{2}-\frac{\ell(l+1)}{2}+k_{s, l} l \\
& =-\widetilde{\tau_{j_{s}}}\left(j_{s}-k_{s, l}+l\right)-\frac{j_{s}-k_{s, l}+l}{2}+\frac{j_{s}^{2}-\left(k_{s, l}-l\right)^{2}}{2} \\
& =-\left(\check{\tau_{j_{s}}}+\frac{1}{2}\right)\left(j_{s}-k_{s, l}+l\right)+\frac{\left(j_{s}+k_{s, l}-l\right)\left(j_{s}-k_{s, l}+l\right)}{2} .
\end{aligned}
$$


Thus

$$
\frac{c t(\mu)-c t(\tau)}{|\mu|-|\tau|}=-\widetilde{\tau_{j_{s}}}-\frac{1}{2}+\frac{j_{s}+k_{s, l}-l}{2} .
$$

Recall that we also have, by definition:

$$
\frac{f(\mu)-f(\tau)}{|\mu|-|\tau|}=\frac{|\mu|+|\tau|-1}{2}+\frac{c t(\mu)-c t(\tau)}{|\mu|-|\tau|}
$$

So

$$
\begin{aligned}
& \frac{f(\mu)-f(\tau)}{|\mu|-|\tau|}=\frac{|\mu|+|\tau|-1}{2}-\check{\tau_{j_{s}}}-\frac{1}{2}+\frac{j_{s}+k_{s, l}-l}{2} \\
& =\frac{2|\tau|+j_{s}-k_{s, l}+l-1}{2}-\check{\tau_{j_{s}}}-\frac{1}{2}+\frac{j_{s}+k_{s, l}-l}{2} \\
& =|\tau|-1+j_{s}-\check{\tau_{j_{s}}}=s .
\end{aligned}
$$

These constructions are compatible with the constructions described in Section 9 in the following sense:

Proposition 10.0.21. Let $n>>0$. Put $\lambda^{(l)}:=\operatorname{rec}\left(l, \operatorname{core}_{(\nu-s)}(\tau)\right)$. Then $\widetilde{\lambda^{(l)}}(n)=\operatorname{rec}\left(l, \operatorname{core}_{(n-s)}(\tilde{\tau}(n))\right)$.

Proof. One can easily see that the procedure for constructing $\operatorname{rec}\left(l, \operatorname{core}_{(\nu-s)}(\tau)\right)$ coincides with the procedure for constructing $\operatorname{rec}\left(l, \operatorname{core}_{(n-s)}(\tilde{\tau}(n))\right)$ and then removing the top row.

\section{Blocks IN THE CATEGORY $\underline{\mathcal{O}}_{c, \nu}$}

11.1. Morphisms between two Verma objects. We now give some necessary and some sufficient conditions for the existence of a non-trivial morphism between Verma objects. Fix Young diagrams $\tau, \mu$.

The purpose of this section is to prove the main theorem:

Theorem 11.1.1. For two distinct Young diagrams $\mu, \tau$ and an integer $m>0$, the following are equivalent:

(1) $|\mu| \neq|\tau|$ and $\mathcal{L}_{\tau, \mu, m} \subset \mathcal{B}_{\mu, \tau}$,

(2) $\mu=\Gamma(\tau, s, \operatorname{sign}(|\mu|-|\tau|))$ for some $s \in C_{\tau}$ (in particular, $\left.|\mu| \neq|\tau|\right)$, and $(|\mu|-|\tau|) \mid m$.

We begin by proving the following proposition (this shows that $(2) \Rightarrow(1)$ in the theorem above):

Proposition 11.1.2. Let $r \in \mathbb{Z} \backslash\{0\}, s \in C_{\tau}$ (i.e. $s=|\tau|-1+j_{s}-\check{\tau}_{j_{s}}$ for some $\left.j_{s} \geq 1\right)$. Assume $\widetilde{\tau_{s}}+\operatorname{sign}(r) \geq 0$.

Let $\mu=\Gamma(\tau, s, \operatorname{sign}(r))$.

If $c^{\prime}=\frac{\nu-s}{r}$, then there exists a non-trivial morphism $M_{c, \nu}(\mu) \longrightarrow M_{c, \nu}(\tau)$.

Proof. Assume $\left(c^{\prime}, \nu\right), \mu$ are as above.

Let $n>>0$ be an integer, such that $\operatorname{gcd}(n-s, r)=1$ (there are infinitely many such positive integers).

By Corollary 9.3.9. Proposition 9.3.2 and Proposition 10.0.21, we have a nontrivial morphism $M_{c, n}(\tilde{\mu}(n)) \longrightarrow M_{c, n}(\tilde{\tau}(n))$. Moreover, $s=\frac{f(\mu)-f(\tau)}{|\mu|-|\tau|}$ ( $f$ defined in Subsection 8.2) by Lemma 10.0.20, and the image of $\tilde{\mu}(n)$ sits in degree $m:=$ $r(|\mu|-|\tau|)$ of $M_{c, n}(\tilde{\tau}(n))$ (this is a direct consequence of equation (8.2.1)). 
Then by Lemma 7.0.11 there is a non-trivial morphism $M_{c, \nu=n}(\mu) \rightarrow M_{c, \nu=n}(\tau)$ with the image of $X_{\mu}$ sitting in degree $m=r(|\mu|-|\tau|)$ of $M_{c, \nu=n}(\tau)$, and $s=$ $\frac{f(\mu)-f(\tau)}{|\mu|-|\tau|}$.

Consider the space $V^{m}{ }_{\nu}=\operatorname{Hom}_{\operatorname{Rep}\left(S_{\nu}\right)}\left(X_{\mu}, S^{m} \mathfrak{h}_{0}^{*} \otimes X_{\tau}\right)$ for any $\nu \in \mathbb{C}$. By the construction of Deligne's categories, these spaces are isomorphic for almost every $\nu \in \mathbb{C}$ (in particular, for all $\nu \notin \mathbb{Z}_{+}$). We will denote the locus of such "generic" points $\nu \in \mathbb{C}$ by $A$ (so $|\mathbb{C} \backslash A|<\infty, \mathbb{C} \backslash \mathbb{Z}_{+} \subset A$ ), and write just $V^{m}$ instead of $V^{m}{ }_{\nu}$ whenever $\nu \in A$.

The map $y_{M_{c, \nu}(\tau)}$ from the definition of $\underline{\operatorname{Rep}}\left(H_{c}(\nu)\right)$ defines a polynomial family of maps

$$
\begin{aligned}
Y_{c, \nu}^{m}: V^{m} & \longrightarrow \operatorname{Hom}_{\underline{\operatorname{Rep}}\left(S_{\nu}\right)}\left(\mathfrak{h}_{0} \otimes X_{\mu}, S^{m-1} \mathfrak{h}_{0}^{*} \otimes X_{\tau}\right), \\
\phi & \mapsto y_{M_{c, \nu}(\tau)} \circ\left(\operatorname{Id}_{\mathfrak{h}_{0}} \otimes \phi\right) .
\end{aligned}
$$

It is easy to see that the locus of points $(c, \nu) \in \mathbb{C} \times A$ such that $Y^{m}{ }_{c, \nu}$ is not injective is a Zariski closed subset of $\mathbb{C} \times A$. Denote it by $\mathcal{C}$.

By definition, $\mathcal{C}$ is exactly the locus of points $(c, \nu) \in \mathbb{C} \times A$ such that

$$
\left.y_{M_{c, \nu}(\tau)}\right|_{\mathfrak{h}_{0} \otimes \phi\left(X_{\mu}\right)}=0
$$

for some $\phi \in V^{m} \backslash\{0\}$. The latter condition is equivalent to the existence of a non-trivial morphism $\Theta_{\nu}: M_{c, \nu}(\mu) \longrightarrow M_{c, \nu}(\tau)$ such that $\Theta_{\nu}\left(X_{\mu}\right)$ sits in degree $m$ of $\left.M_{c, \nu}(\tau)\right)$.

The morphisms $M_{c, \nu=n}(\mu) \longrightarrow M_{c, \nu=n}(\tau), n \in \mathbb{Z}_{>>0}$ constructed above guarantee that $Y_{c, \nu=n}^{m}$ is not injective whenever $n \in \mathbb{Z}_{>>0}, c^{\prime}=\frac{1}{c}=\frac{n-s}{r}$, which means that $(c, \nu=n) \subset \mathcal{C}$ for any $c, n$ satisfying $n>>0, \frac{1}{c}=\frac{n-s}{r}$.

Thus $\left\{(c, \nu) \in \mathbb{C}^{2} \mid c^{\prime}=\frac{1}{c}=\frac{\nu-s}{r}\right\} \subset \mathcal{C}$, and we obtain the required statement.

Remark 11.1.3. In the proof of this proposition, we established that $s=\frac{f(\mu)-f(\tau)}{|\mu|-|\tau|}$, so by equation (8.2.1), the image of $X_{\mu} \subset M_{c, \nu}(\mu)$ sits in degree $m$ of $M_{c, \nu}(\tau)$ for all $\left(c^{\prime}, \nu\right)$ such that $c^{\prime}=\frac{\nu-s}{r}$. In general, for each pair $\left(c^{\prime}, \nu\right)$, equation 8.2.1) allows us to compute the unique degree $m$ in which the image of $X_{\mu}$ could sit in $M_{c, \nu}(\tau)$.

We continue with Young diagrams $\tau, \mu$ fixed, and proceed to prove the remainder of Theorem 11.1.1.

Proof of Theorem 11.1.1. We prove the direction $(1) \Rightarrow(2)$ of Theorem 11.1.1.

Assume $|\mu| \neq|\tau|$.

Consider the line $\mathcal{L}_{\tau, \mu, m} \subset \mathbb{C}^{2}$ (defined in Notation 8.1.5). We would like to check whether $\mathcal{L}_{\tau, \mu, m}$ satisfies the following condition:

Condition 11.1.4. For all $\left(c^{\prime}, \nu\right) \in \mathcal{L}_{\tau, \mu, m}$ there exists a non-trivial morphism $\Theta_{\nu}: M_{c, \nu}(\mu) \longrightarrow M_{c, \nu}(\tau)$ such that $\Theta_{\nu}\left(X_{\mu}\right)$ sits in degree $m$ of $M_{c, \nu}(\tau)$.

Assume this is indeed the case. We will show that this implies that $\tau, \mu, m$ satisfy Part (2) of Theorem 11.1.1.

Since $|\mu| \neq|\tau|$, the equation (8.1.1) tells us that for almost any $n \in \mathbb{Z}_{+}$there exists $c^{\prime} \in \mathbb{C}$ such that $\left(c^{\prime}, \nu=n\right) \in \mathcal{L}_{\tau, \mu, m}$.

Due to Lemma 7.0.11. Condition 11.1.4 implies that for integer $n>>0$ there must exist non-trivial morphisms $\tilde{\Theta}_{n}: M_{c, n}(\tilde{\mu}(n)) \longrightarrow M_{c, n}(\tilde{\tau}(n))$ (corresponding to $\left.\Theta_{\nu=n}\right)$ with the image of $\tilde{\mu}(n)$ sitting in degree $m$ of $M_{c, n}(\tilde{\tau}(n))$. 
As we saw in Section 9] this statement imposes a rather strong condition on the Young diagrams $\tau, \mu$, as well as on the integer $m$. The remainder of the proof consists of translating these conditions to the setting of Deligne categories, which will result in the conditions from Part (2) of the statement of Theorem 11.1.1.

Recall that the requirement that $\left(c^{\prime}, n\right) \in \mathcal{L}_{\tau, \mu, m}$ is equivalent to the requirement

$$
c^{\prime}=\frac{(|\mu|-|\tau|) n-(f(\mu)-f(\tau))}{m} .
$$

Denote:

$$
a:=|\mu|-|\tau|, b:=f(\mu)-f(\tau), d_{n}:=\operatorname{gcd}(a n-b, m) .
$$

Then

$$
c^{\prime}=\frac{a n-b}{m}=\frac{(a n-b) / d_{n}}{m / d_{n}}
$$

where

$$
\frac{a n-b}{d_{n}} \in \mathbb{Z}, \frac{m}{d_{n}} \in \mathbb{Z}_{>0}, \operatorname{gcd}\left(\frac{a n-b}{d_{n}}, \frac{m}{d_{n}}\right)=1 .
$$

By Theorem 9.3.1, if $(a n-b) / d_{n} \neq 2$, we can pass to the case $\left(c^{\prime}=(a n-b) / d_{n}, n\right)$, with the image of $\tilde{\mu}(n)$ sitting in degree $d_{n}$ of $M_{c, n}(\tilde{\tau}(n))$.

Note that since $a \neq 0$ and since $0<d_{n} \leq m$, we have $(a n-b) / d_{n} \neq 2$ for $n>>0$.

Now we have the following cases:

Case $a>0$.

Lemma 11.1.5. For $n>>0$, if there are no non-trivial morphisms $\tilde{\Theta}_{n}$ then $a|b, a| m$, i.e. $d_{n}=a$.

Proof. Assume there are non-trivial morphisms $\tilde{\Theta}_{n}$ for $n>>0$. Then we have, by Theorem 9.3.5.

$$
\frac{a n-b}{d_{n}}<n
$$

for $n>>0$; that is, $a \leq d_{n}$. Also, putting

$$
d:=\operatorname{gcd}(a, b), a^{\prime}:=\frac{a}{d}, b^{\prime}:=\frac{b}{d}
$$

we have $d_{n}=\operatorname{gcd}\left(d\left(a^{\prime} n-b^{\prime}\right), m\right) \geq a=d a^{\prime} \geq 1$ for $n>>0$. This implies, for $n>>0$ : either $d_{n} \mid d$ (possible only if $d_{n}=d=a$, and then we have $a|b, a| m$ ), or $\operatorname{gcd}\left(a^{\prime} n-b^{\prime}, m\right) \neq 1$.

So we only need to check that there is no $n_{0} \in \mathbb{Z}$ such that $\forall n>n_{0}, \operatorname{gcd}\left(a^{\prime} n-\right.$ $\left.b^{\prime}, m\right) \neq 1$. Indeed, $b^{\prime}, \operatorname{gcd}\left(a^{\prime}, m\right)$ are relatively prime (since $a^{\prime}, b^{\prime}$ are), and so $\operatorname{gcd}\left(a^{\prime} n-b^{\prime}, m\right)=\operatorname{gcd}\left(\frac{a^{\prime}}{\operatorname{gcd}\left(a^{\prime}, m\right)} n-b^{\prime}, \frac{m}{\operatorname{gcd}\left(a^{\prime}, m\right)}\right)$. Now

$$
\operatorname{gcd}\left(\frac{a^{\prime}}{\operatorname{gcd}\left(a^{\prime}, m\right)}, b^{\prime}\right)=\operatorname{gcd}\left(\frac{a^{\prime}}{\operatorname{gcd}\left(a^{\prime}, m\right)}, \frac{m}{d^{\prime}}\right)=1
$$

SO

$$
\operatorname{gcd}\left(a^{\prime} n-b^{\prime}, m\right)=\operatorname{gcd}\left(\frac{a^{\prime}}{\operatorname{gcd}\left(a^{\prime}, m\right)} n-b^{\prime}, \frac{m}{\operatorname{gcd}\left(a^{\prime}, m\right)}\right)=1
$$

for infinitely many integer values of $n$. 
Thus $d_{n}=a$, and $(a n-b) / d_{n} \neq 2$ for $n>>0$. So to understand what happens for $\left(c^{\prime}, n\right) \in \mathcal{L}_{\tau, \mu, m}$, it is enough to check what happens for the case $\left(c^{\prime}=n-(b / a), n\right)$ (recall: $b / a=\frac{f(\mu)-f(\tau)}{|\mu|-|\tau|} \in \mathbb{Z}$ ). Still assuming the existence of non-trivial morphisms $\tilde{\Theta}_{n}$, Proposition 10.0 .21 and Corollary 9.3 .9 give:

For $n>>0$ and $c^{\prime}=n-b / a$, put $s:=b / a$, and we have

$$
\mu=\Gamma(\tau, s, 1) \text { for } j_{s} \geq 1, \text { such that } s=|\tau|-1+j_{s}-\check{\tau}_{j_{s}} .
$$

Note that a priori, $j_{s}$ could depend on $n$, but the equality $s=|\tau|-1+j_{s}-\check{\tau_{j}}$ defines $j_{s}$ uniquely for each $s$, so since $s$ doesn't depend on $n$, neither does $j_{s}$.

Conclusion 11.1.6. For $|\mu|>|\tau|, \mathcal{L}_{\tau, \mu, m}$ satisfies Condition 11.1.4 if and only if the following hold:

(1) $s:=\frac{f(\mu)-f(\tau)}{|\mu|-|\tau|} \in C_{\tau} \subset \mathbb{Z}$,

(2) $\mu=\Gamma(\tau, s, 1)$,

(3) $(|\mu|-|\tau|) \mid m$.

In that case, points $\left(c^{\prime}, \nu\right) \in \mathcal{L}_{\tau, \mu, m}$ satisfy the condition: $c^{\prime}=\frac{\nu-s}{r}$ for $r=\frac{m}{|\mu|-|\tau|}$.

Case $a<0$.

By Observation 9.3.2 the existence of a non-trivial morphism $\tilde{\Theta}_{n}$ for $n>>0$ is equivalent to the existence of a non-trivial morphism $\tilde{\Upsilon}_{n}: M_{-c^{\prime}, n}(\tilde{\tau}(n)) \longrightarrow$ $M_{-c^{\prime}, n}(\tilde{\mu}(n))$. The necessary and sufficient conditions for the existence of a nontrivial morphism $\tilde{\Upsilon}_{n}$ for all $n>>0$ are given in Conclusion 11.1.6, with $\mu$ and $\tau$ switching roles.

Conclusion 11.1.7. For $|\mu|<|\tau|, \mathcal{L}_{\tau, \mu, m}$ satisfies Condition 11.1.4 if and only if the following hold:

(1) $s:=\frac{f(\mu)-f(\tau)}{|\mu|-|\tau|} \in C_{\mu} \subset \mathbb{Z}$,

(2) $\mu=\Gamma(\tau, s,-1)$,

(3) $(|\tau|-|\mu|) \mid m$.

In that case, points $\left(c^{\prime}, \nu\right) \in \mathcal{L}_{\tau, \mu, m}$ satisfy the condition: $c^{\prime}=-\frac{\nu-s}{r}$ for $r=$ $\frac{m}{|\tau|-|\mu|}$.

Remark 11.1.8. The results in this subsection agree with the statement of Lemma 8.1 .9 when $m=1$, but they imply the statement of Lemma 8.1.9 only if $|\mu| \neq|\tau|$.

11.2. Properties of lines $\mathcal{L}_{\tau, \mu, m}$. We conclude this section with a list of (almost trivial) properties of the lines $\mathcal{L}_{\tau, \mu, m}$ :

Lemma 11.2.1. Let $m_{1}, m_{2} \in \mathbb{Z}_{+}$, and let $\tau, \mu, \mu^{(1)}, \mu^{(2)}$ be Young diagrams. Then

- $\mathcal{L}_{\tau, \mu, m_{1}} \cap \mathcal{L}_{\tau, \mu, m_{2}} \neq \emptyset \Leftrightarrow m_{1}=m_{2}$.

- If $\left(c^{\prime}, \nu\right) \in \mathcal{L}_{\tau, \mu^{(1)}, m_{1}} \cap \mathcal{L}_{\tau, \mu^{(2)}, m_{2}}$, and the lines $\mathcal{L}_{\tau, \mu^{(1)}, m_{1}}, \mathcal{L}_{\tau, \mu^{(2)}, m_{2}}$ do not coincide, then $c^{\prime}, \nu \in \mathbb{Q}$ (and there is only one point $\left(c^{\prime}, \nu\right)$ like this).

- Assume the lines $\mathcal{L}_{\tau, \mu^{(1)}, m_{1}}, \mathcal{L}_{\tau, \mu^{(2)}, m_{2}}$ coincide, $\left|\mu^{(1)}\right| \neq|\tau|$, and $\mathcal{L}_{\tau, \mu^{(1)}, m_{1}}$ $\subset \mathcal{B}_{\mu^{(1)}, \tau}$. Then $\mathcal{L}_{\tau, \mu^{(2)}, m_{2}} \subset \mathcal{B}_{\mu^{(2)}, \tau}$ iff $\mu^{(1)}=\mu^{(2)}$.

Proof.

- Follows immediately from the definition of $\mathcal{L}_{\tau, \mu, m}$ (see Notation 8.1.5).

- Follows immediately from the definition of $\mathcal{L}_{\tau, \mu, m}$ by a linear equation with rational coefficients. 
- First, $\left|\mu^{(1)}\right| \neq|\tau|$, and $\mathcal{L}_{\tau, \mu^{(1)}, m_{1}} \subset \mathcal{B}_{\mu^{(1)}, \tau}$, so by Theorem 11.1.1, putting

$$
s:=\frac{f\left(\mu^{(1)}\right)-f(\tau)}{\left|\mu^{(1)}\right|-|\tau|}
$$

we obtain

$$
\mu^{(1)}=\Gamma\left(\tau, s, \operatorname{sign}\left(\left|\mu^{(1)}\right|-|\tau|\right)\right)
$$

with $j_{s}$ given by $s=|\tau|-1+j_{s}-\widetilde{\tau_{j}}$.

Next, since the lines $\mathcal{L}_{\tau, \mu^{(1)}, m_{1}}, \mathcal{L}_{\tau, \mu^{(2)}, m_{2}}$ coincide and $\left|\mu^{(1)}\right| \neq|\tau|$, we obtain:

$$
\left[m_{1}:\left(\left|\mu^{(1)}\right|-|\tau|\right): f\left(\mu^{(1)}\right)-f(\tau)\right]=\left[m_{2}:\left(\left|\mu^{(2)}\right|-|\tau|\right): f\left(\mu^{(2)}\right)-f(\tau)\right]
$$

which implies $s=\frac{f\left(\mu^{(1)}\right)-f(\tau)}{\left|\mu^{(1)}\right|-|\tau|}=\frac{f\left(\mu^{(2)}\right)-f(\tau)}{\left|\mu^{(2)}\right|-|\tau|}$.

We also get: $\left|\mu^{(2)}\right| \neq|\tau|$ and $\operatorname{sign}\left(\left|\mu^{(1)}\right|-|\tau|\right)=\operatorname{sign}\left(\left|\mu^{(2)}\right|-|\tau|\right)$.

Now assume that $\mathcal{L}_{\tau, \mu^{(2)}, m_{2}} \subset \mathcal{B}_{\mu^{(2)}, \tau}$. Since

$$
\left|\mu^{(2)}\right| \neq|\tau|, s=\frac{f\left(\mu^{(1)}\right)-f(\tau)}{\left|\mu^{(1)}\right|-|\tau|}=\frac{f\left(\mu^{(2)}\right)-f(\tau)}{\left|\mu^{(2)}\right|-|\tau|}
$$

and

we obtain, from 11.1.1

$$
\operatorname{sign}\left(\left|\mu^{(1)}\right|-|\tau|\right)=\operatorname{sign}\left(\left|\mu^{(2)}\right|-|\tau|\right)
$$

$$
\mu^{(2)}=\Gamma\left(\tau, s, \operatorname{sign}\left(\left|\mu^{(2)}\right|-|\tau|\right)\right)=\mu^{(1)} .
$$

From the third part of the above lemma, we immediately get:

Corollary 11.2.2. Let $|\mu| \neq|\tau|$, and $\mathcal{L}_{\tau, \mu, m} \subset \mathcal{B}_{\mu, \tau}$. Then for a generic point $\left(c^{\prime}, \nu\right) \in \mathcal{L}_{\tau, \mu, m},\left(c^{\prime}, \nu\right) \notin \mathcal{B}_{\mu^{\prime}, \tau}$ for any $\mu^{\prime} \neq \mu$.

\section{Characters of Simple objects in $\underline{\mathcal{O}}_{c, \nu}$}

Throughout this section, we assume that $\nu \notin \mathbb{Z}_{+}, c \neq 0$. We also continue to identify $\mathfrak{h}_{0}$ with $\mathfrak{h}_{0}^{*}, \mathfrak{h}$ with $\mathfrak{h}^{*}$.

\subsection{Definitions.}

Definition 12.1.1. The character of a graded vector space $V=\bigoplus_{k \in \mathbb{Z}} V_{k}$ will be defined as $c h_{q}(V):=\sum_{k} q^{k} \operatorname{dim} V_{k}$.

Definition 12.1.2 (Character of a graded ind-object of $\operatorname{Rep}\left(S_{\nu}\right)$ ). The character of an ind-object $V$ of $\underline{\operatorname{Rep}}\left(S_{\nu}\right)$ with the grading $V=\bigoplus_{j \in \mathbb{Z}_{+}} V_{j}, V_{j} \in \underline{\operatorname{Rep}}\left(S_{\nu}\right)$ is defined to be a formal power series in $t$ :

$$
\operatorname{ch}_{t} V=\sum_{\mu \in \mathcal{P}} X_{\mu} \operatorname{ch}_{t}\left(\operatorname{Hom}_{S_{\nu}}\left(X_{\mu}, V\right)\right) .
$$

Definition 12.1.3 (Character of a object of $\underline{\mathcal{O}}_{c, \nu}$ ). Let $M$ be an object of the category $\underline{\mathcal{O}}_{c, \nu}$. The character of $M$ is defined to be a formal power series in $t$ :

$$
c h_{t} M:=\sum_{\mu \in \mathcal{P}} X_{\mu} \operatorname{tr}\left(\left.t^{\boldsymbol{h}}\right|_{\operatorname{Hom}_{S_{\nu}}\left(X_{\mu}, M\right)}\right)
$$

with complex degrees, and coefficients in $K_{0}\left(\underline{\operatorname{Rep}}\left(S_{\nu}\right)\right)$, which is the Grothendieck ring of $\underline{\operatorname{Rep}}\left(S_{\nu}\right)$. We will usually write $c h M$ instead of $c h_{t} M$ for short. 
Remark 12.1.4. The trace of the operator $t^{\boldsymbol{h}}$ is defined since $\boldsymbol{h}$ acts locally finitely on objects in $\underline{\mathcal{O}}_{c, \nu}$.

12.2. Characters of Verma objects. Recall that $M(\lambda) \cong S \mathfrak{h}_{0} \otimes X_{\lambda}$ as Indobjects of $\operatorname{Rep}\left(S_{\nu}\right)$. So it is enough to compute the character of $S \mathfrak{h}_{0}$, and then use the formula

$$
\operatorname{ch}_{t} M(\lambda)=\operatorname{ch}_{t}\left(S \mathfrak{h}_{0}\right) X_{\lambda} t^{h_{c, \nu}(\lambda)} .
$$

We now give a formula for computing the character of the ind-object $S \mathfrak{h}_{0}$ of $\underline{\operatorname{Rep}}\left(S_{\nu}\right)$, which comes from the character formula in [10, 3.7].

Proposition 12.2.1. We have the following formula for the character of $S \mathfrak{h}_{0}$ :

$$
\operatorname{ch}_{t}\left(S \mathfrak{h}_{0}\right)=\frac{1}{\sum_{n \geq 0}(-1)^{n} \Lambda^{n} \mathfrak{h}_{0} t^{n}} .
$$

Proof. Consider the category Schur of all Schur functors: the objects are Schur functors

$$
S_{R}:(\text { FinVect }) \rightarrow(\text { FinVect }), S_{R}(\cdot)=\bigoplus_{n \geq 0} R_{n} \otimes_{\mathbb{C}\left[S_{n}\right]}(\cdot)^{\otimes n}
$$

where $R=\left(R_{n}\right)_{n \geq 0}$, each $R_{n}$ being a finite-dimensional representation of $S_{n}$, and only finitely many of these representations being nonzero; the morphisms are natural transformations between such functors. This category is equivalent to the category $\bigoplus_{n \in \mathbb{Z}} \operatorname{Rep}\left(S_{n}\right)$, and thus semisimple, with the simple objects parameterized (up to isomorphism) by the set of all Young diagrams.

The tensor structure on Schur comes from the tensor structure on (FinVect). Namely, given two simple objects parametrized by Young diagrams $\lambda$ and $\mu$, their tensor product decomposes as a direct sum of simple objects $\rho$, with the multiplicity of $\rho$ being the Littlewood-Richardson coefficient $c_{\lambda, \mu}^{\rho}$.

One can see that $\operatorname{Fun}\left(\operatorname{Schur}, \operatorname{Rep}\left(S_{\nu}\right)\right) \cong \operatorname{Rep}\left(S_{\nu}\right)$ (this is true for any symmetric tensor category), where Fun $\left(S c \overline{h u r}, \operatorname{Rep}\left(S_{\nu}\right)\right)$ are tensor functors between additive linear tensor categories.

Fix the object $V$ in Schur corresponding to the identity functor (which is, of course, a Schur functor as well). Then we can identify the simple objects in Schur with $S^{\lambda} V$ (the Schur functor $S^{\lambda}$ applied to $V$ ), where $\lambda$ runs through all Young diagrams.

Consider the commutative algebra $S V$, and the exterior power $\Lambda^{m} V(m \geq 0)$. Then we have (cf. [12, 6.8]):

$$
S V \otimes \Lambda^{m} V=\bigoplus_{\lambda \in \mathcal{I}_{\pi^{m}}^{+}} S^{\lambda} V
$$

where $\mathcal{I}_{\pi^{m}}^{+}$is the set of all Young diagrams obtained from $\pi^{m}$ by adding several boxes, no two in the same column.

One can easily see that for any $m \geq 1$,

$$
\mathcal{I}_{\pi^{m}}^{+}=\left(\mathcal{I}_{\pi^{m+1}}^{+} \cap \mathcal{I}_{\pi^{m}}^{+}\right) \sqcup\left(\mathcal{I}_{\pi^{m-1}}^{+} \cap \mathcal{I}_{\pi^{m}}^{+}\right)
$$

and $\mathcal{I}_{\pi^{0}}^{+}=\mathcal{I}_{\emptyset}^{+}=\mathcal{I}_{\pi^{1}}^{+} \sqcup\{\emptyset\}$. So we have an infinite exact sequence in Schur $\cong$ $\bigoplus_{n \in \mathbb{Z}} \operatorname{Rep}\left(S_{n}\right)$ :

$\ldots \longrightarrow S V \otimes \Lambda^{m} V \longrightarrow S V \otimes \Lambda^{m-1} V \longrightarrow \ldots \longrightarrow S V \otimes V \longrightarrow S V \longrightarrow S^{\emptyset} V \longrightarrow 0$ 
Notice that when applying these functors to a finite-dimensional vector space $W$, we obtain a finite exact sequence which is the Koszul resolution of $\mathbb{C}$ over the symmetric algebra $S W$.

Thus we obtain an exact complex of Ind-objects of $\operatorname{Rep}\left(S_{\nu}\right)$ :

$\cdots \longrightarrow S \mathfrak{h}_{0} \otimes \Lambda^{m} \mathfrak{h}_{0} \longrightarrow S \mathfrak{h}_{0} \otimes \Lambda^{m-1} \mathfrak{h}_{0} \longrightarrow \cdots \longrightarrow S \mathfrak{h}_{0} \otimes \mathfrak{h}_{0} \longrightarrow S \mathfrak{h}_{0} \longrightarrow \mathbb{1} \longrightarrow 0$

Now the character formula follows directly from Euler's formula applied to this complex.

12.3. The graded space $\operatorname{Hom}_{\underline{\operatorname{Rep}}\left(S_{\nu}\right)}\left(X_{\mu}, S \mathfrak{h}_{0} \otimes X_{\tau}\right)$. In this subsection we describe explicitly the decomposition of $S \mathfrak{h} \otimes X_{\tau}$ and $S \mathfrak{h}_{0} \otimes X_{\tau}$ as $\operatorname{Rep}\left(S_{\nu}\right)$ ind-objects into graded sums of simple objects.

Lemma 12.3.1. For $n>>0$ (in fact, for $n>2(|\mu|+|\tau|)$ ), we have:

$$
c h_{q}\left(\operatorname{Hom}_{S_{n}}\left(\tilde{\mu}(n), \mathbb{C}\left[x_{1}, \ldots, x_{n}\right] \otimes \tilde{\tau}(n)\right)\right)=\left(s_{\tilde{\tau}(n)} * s_{\tilde{\mu}(n)}\right)\left(1, q, q^{2}, \ldots\right) .
$$

The latter expression can be rewritten as

$$
\begin{aligned}
& \operatorname{ch}_{q}\left(\operatorname{Hom}_{S_{n}}\left(\tilde{\mu}(n), \mathbb{C}\left[x_{1}, \ldots, x_{n}\right] \otimes \tilde{\tau}(n)\right)\right)=\sum_{\lambda,|\lambda|+\lambda_{1} \leq n} \gamma_{\tilde{\tau}(n), \tilde{\mu}(n)}^{\tilde{\lambda}(n)} s_{\tilde{\lambda}(n)}\left(1, q, q^{2}, \ldots\right) \\
& =\sum_{\lambda} \bar{\gamma}_{\tau, \mu}^{\lambda} s_{\tilde{\lambda}(n)}\left(1, q, q^{2}, \ldots\right) .
\end{aligned}
$$

Here

- $s_{\tilde{\lambda}(n)}$ is the Schur symmetric function corresponding to the partition $\tilde{\lambda}(n)$ of $n$ (see [18, Chapter I, Par. 3, p. 41]),

- $s_{\tilde{\tau}(n)} * s_{\tilde{\mu}(n)}$ is the internal product of Schur symmetric functions as defined in [18, Chapter I, Par. 7, p. 116],

- $\gamma_{\alpha^{\prime \prime}, \alpha^{\prime \prime \prime}}^{\alpha^{\prime}}:=\frac{1}{n !} \sum_{w \in S_{n}} \chi^{\alpha^{\prime}}(w) \chi^{\alpha^{\prime \prime}}(w) \chi^{\alpha^{\prime \prime \prime}}(w)$ is the Kronecker coefficient of partitions $\alpha^{\prime}, \alpha^{\prime \prime}, \alpha^{\prime \prime \prime}$ of $n$ (here $\chi^{\alpha^{\prime}}(w)$ is the value at $w \in S_{n}$ of the character of the irreducible representation of $S_{n}$ corresponding to the Young diagram $\left.\alpha^{\prime}\right)$.

- $\bar{\gamma}_{\tau, \mu}^{\lambda}$ is the reduced Kronecker coefficient (equals $\gamma_{\tilde{\tau}(n), \tilde{\mu}(n)}^{\tilde{\lambda}(n)}$ for $n>>0$ ). A good reference for standard and reduced Kronecker coefficients is 4 .

Proof. We define the map $\mathcal{F}$ (denoted by ch in [18, Chapter I, Par. 7]) from conjugation-invariant functions on $S_{n}$ to symmetric functions in countably many variables by putting

$$
\mathcal{F}(f):=\frac{1}{n !} \sum_{w \in S_{n}} f(w) \prod_{j \geq 1}\left(\sum_{i} x_{i}^{\rho_{j}(w)}\right)
$$

where $\rho(w)=\left(\rho_{1}, \rho_{2}, \ldots\right)$ is the cycle-type of $w$.

Denoting

$$
p_{\rho}:=\prod_{j \geq 1}\left(\sum_{i} x_{i}^{\rho_{j}}\right), m(\rho)_{i}:=\text { number of parts of size } i \text { in } \rho, z_{\rho}:=\prod_{i \geq 1} i^{m(\rho)_{i}} m(\rho)_{i} \text { ! }
$$

we obtain

$$
\mathcal{F}(f):=\sum_{\rho \vdash n} \frac{1}{z_{\rho}} f(\rho) p_{\rho}
$$


(here $f(\rho)$ is the value of $f$ on the conjugacy class of $S_{n}$ consisting of permutations of cycle-type $\rho$ ).

Let $V$ be a representation of $S_{n}$. Denote by $\chi^{V}$ the character of $V$, and by abuse of notation, $\mathcal{F}(V):=\mathcal{F}\left(\chi^{V}\right)$. If $V=\bigoplus_{j} V_{j}$ is a $\mathbb{Z}$-graded representation of $S_{n}$, then put $\mathcal{F}_{q}(V):=\sum_{j \in \mathbb{Z}} \mathcal{F}\left(\chi^{V_{j}}\right) q^{j}$.

We have: $\mathcal{F}\left(\chi^{\alpha}\right)=s_{\alpha}$ for partition $\alpha$ of $n$, and $\mathcal{F}\left(\chi^{V^{\prime}} \chi^{V^{\prime \prime}}\right)=: \mathcal{F}\left(V^{\prime}\right) * \mathcal{F}\left(V^{\prime \prime}\right)$ $\left(\chi^{V^{\prime}} \chi^{V^{\prime \prime}}\right.$ is the character of the representation $\alpha^{\prime} \otimes \alpha^{\prime \prime}$ of $\left.S_{n}\right)$.

Denote by $g_{w}$ the action of $w \in S_{n}$ on the $n$-dimensional complex vector space $\mathbb{C}^{n}, g_{w}$ given by the permutation matrix corresponding to $w$.

By MacMahon's Master theorem (see [10, Lemma 3.28]; the proof relies on an argument similar to the one used in 12.2), we have:

$$
\begin{aligned}
& \mathcal{F}_{q}\left(\mathbb{C}\left[x_{1}, \ldots, x_{n}\right]\right)=\frac{1}{n !} \sum_{w \in S_{n}} \sum_{k \geq 0} \operatorname{tr}\left(\operatorname{Sym}^{k}\left(g_{w}\right)\right) q^{k} p_{\rho(w)}=\frac{1}{n !} \sum_{w \in S_{n}} \frac{1}{\operatorname{det}\left(1-q g_{w}\right)} p_{\rho(w)} \\
& =\frac{1}{n !} \sum_{w \in S_{n}} \prod_{1 \leq k \leq \ell(\rho(w))} \frac{1}{\left(1-q^{\left.\rho(w)_{k}\right)}\right.} p_{\rho(w)}=\sum_{\rho \vdash n} \frac{1}{z_{\rho}} \prod_{1 \leq k \leq \ell(\rho)} \frac{1}{\left(1-q^{\rho_{k}}\right)} p_{\rho} .
\end{aligned}
$$

Similarly,

$$
\begin{aligned}
& \left.\mathcal{F}_{q}\left(\mathbb{C}\left[x_{1}, \ldots, x_{n}\right] \otimes \tilde{\tau}(n)\right)\right)=\sum_{\rho \vdash n} \frac{1}{z_{\rho}} \prod_{1 \leq k \leq \ell(\rho)} \frac{1}{\left(1-q^{\rho_{k}}\right)} p_{\rho} \chi^{\tilde{\tau}(n)}(\rho) \\
& =\sum_{\alpha, \rho \vdash n} \frac{1}{z_{\rho}} \prod_{1 \leq k \leq \ell(\rho)} \frac{1}{\left(1-q^{\rho_{k}}\right)} \chi^{\alpha}(\rho) \chi^{\tilde{\tau}(n)}(\rho) s_{\alpha} .
\end{aligned}
$$

Thus,

$$
\begin{aligned}
& c h_{q}\left(\operatorname{Hom}_{S_{n}}\left(\tilde{\mu}(n), \mathbb{C}\left[x_{1}, \ldots, x_{n}\right] \otimes \tilde{\tau}(n)\right)\right)=\text { coefficient of } s_{\tilde{\mu}(n)} \\
& \left.\quad \operatorname{in~} \mathcal{F}_{q}\left(\mathbb{C}\left[x_{1}, \ldots, x_{n}\right] \otimes \tilde{\tau}(n)\right)\right) \\
& =\sum_{\rho \vdash n} \frac{1}{z_{\rho}} \prod_{1 \leq k \leq \ell(\rho)} \frac{1}{\left(1-q^{\rho_{k}}\right)} \chi^{\tilde{\mu}(n)}(\rho) \chi^{\tilde{\tau}(n)}(\rho)=\left(s_{\tilde{\tau}(n)} * s_{\tilde{\mu}(n)}\right)\left(1, q, q^{2}, \ldots\right) .
\end{aligned}
$$

The last equality holds by [18, Chapter 6, par. 8, p. 363].

Corollary 12.3.2. Taking $\mathfrak{h}_{0}$ to be the reflection representation of $S_{n}$, we obtain:

$$
\begin{aligned}
\operatorname{ch}_{q}\left(\operatorname{Hom}_{S_{n}}\left(\tilde{\mu}(n), S \mathfrak{h}_{0} \otimes \tilde{\tau}(n)\right)\right) & =(1-q)\left(s_{\tilde{\tau}(n)} * s_{\tilde{\mu}(n)}\right)\left(1, q, q^{2}, \ldots\right) \\
& =(1-q) \sum_{\lambda} \bar{\gamma}_{\tau, \mu}^{\lambda} s_{\tilde{\lambda}(n)}\left(1, q, q^{2}, \ldots\right) .
\end{aligned}
$$

Proof. This follows directly from the fact that $\mathbb{C}\left[x_{1}, \ldots, x_{n}\right]=S \mathfrak{h}$, where $\mathfrak{h}=\mathfrak{h}_{0} \oplus \mathbb{C}$ is the permutation representation of $S_{n}$, and so $S^{m} \mathfrak{h}=\bigoplus_{0 \leq j \leq m} S^{j} \mathfrak{h}_{0}$.

\section{Corollary 12.3.3.}

$$
c h_{q}\left(\operatorname{Hom}_{S_{\nu}}\left(X_{\mu}, S \mathfrak{h}_{0} \otimes X_{\tau}\right)\right)=(1-q) \sum_{\lambda \in \mathcal{P}} \bar{\gamma}_{\tau, \mu}^{\lambda} \bar{s}_{\lambda}\left(1, q, q^{2}, \ldots\right)
$$

where $\bar{\gamma}_{\tau, \mu}^{\lambda}$ is the reduced Kronecker coefficient, and

$$
\bar{s}_{\lambda}\left(1, q, q^{2}, \ldots\right):=\frac{q^{|\lambda|} s_{\lambda}\left(1, q, q^{2}, \ldots\right)}{\prod_{j \geq 1}\left(1-q^{j}\right)} .
$$


Proof. By the structure of Deligne's category described in Section 4 if there exist integers $k, N$ such that for any $n \geq N$, $\operatorname{dim} \operatorname{Hom}_{S_{n}}\left(\tilde{\mu}(n), S^{m} \mathfrak{h}_{0}\right)=k$ (here for each $n, \mathfrak{h}_{0}$ is the reflection representation of $\left.S_{n}\right)$, then $\operatorname{dim} \operatorname{Hom}_{S_{\nu}}\left(X_{\mu}, S^{m} \mathfrak{h}_{0}\right)=k$ in Deligne's category $\operatorname{Rep}\left(S_{\nu}\right)$ as well.

Thus the power series in $c h_{q}\left(\operatorname{Hom}_{S_{\nu}}\left(X_{\mu}, S \mathfrak{h}_{0} \otimes X_{\tau}\right)\right)$ is the $q$-adic limit, as $n$ tends to infinity, of $c h_{q}\left(\operatorname{Hom}_{S_{n}}\left(\tilde{\mu}(n), S \mathfrak{h}_{0} \otimes \tilde{\tau}(n)\right)\right)$; recall that, by definition, a sequence $\left\{P^{(n)}(q)\right\}_{n}, P^{(n)}(q)=\sum_{k \geq 0} a_{k}^{(n)} q^{k}$ of formal power series in $q$ converges, in the $q$-adic metric, to the formal power series $P(q)$ if for any $k \geq 0$ there exists $N_{k} \in \mathbb{Z}_{>0}$ such that for any $n>N_{k}, q^{k+1} \mid\left(P(q)-P^{(n)}(q)\right)$.

By [18, Chapter I, Par. 5, Example 1],

$$
s_{\lambda}\left(1, q, q^{2}, \ldots\right)=\frac{q^{\sum_{i \geq 1} i \lambda_{i}-|\lambda|}}{\prod_{x \in \lambda}\left(1-q^{h(x)}\right)}
$$

where $h(x)$ is the size of the hook in $\lambda$ with vertex $x$, so

$$
s_{\tilde{\lambda}(n)}\left(1, q, q^{2}, \ldots\right)=\frac{q^{\sum_{i} i \lambda_{i}}}{\prod_{x \in \lambda}\left(1-q^{h(x)}\right)} \cdot \prod_{1 \leq j \leq n-|\lambda|} \frac{1}{1-q^{n-|\lambda|-j+1+\lambda_{j}}} .
$$

The $q$-adic limit of $s_{\tilde{\lambda}(n)}\left(1, q, q^{2}, \ldots\right)$, as $n$ tends to infinity, is then

$$
\bar{s}_{\lambda}\left(1, q, q^{2}, \ldots\right):=\frac{q^{\sum_{i} i \lambda_{i}}}{\prod_{x \in \lambda}\left(1-q^{h(x)}\right)} \cdot \frac{1}{\prod_{j \geq 1}\left(1-q^{j}\right)}=\frac{q^{|\lambda|} s_{\lambda}\left(1, q, q^{2}, \ldots\right)}{\prod_{j \geq 1}\left(1-q^{j}\right)} .
$$

This completes the proof of the statement.

Example 12.3.4. For $\tau=\emptyset$, we obtain:

$$
\begin{aligned}
& c h_{q}\left(\operatorname{Hom}_{S_{\nu}}\left(X_{\mu}, S \mathfrak{h}_{0}\right)\right)=(1-q) \bar{s}_{\mu}\left(1, q, q^{2}, \ldots\right)=\frac{q^{|\mu|} s_{\mu}\left(1, q, q^{2}, \ldots\right)}{\prod_{j \geq 2}\left(1-q^{j}\right)} \\
& =\frac{q^{\sum_{i \geq 1} \mu_{i} i}}{\prod_{x \in \mu}\left(1-q^{h(x)}\right) \prod_{j \geq 2}\left(1-q^{j}\right)}
\end{aligned}
$$

\subsection{Characters of simple objects: Generic cases.}

12.4.1. Fix a Young diagram $\tau$. Recall that for each Young diagram $\mu \neq \tau$ and $m \in \mathbb{Z}_{+}$, there is a set of points $\left(c^{\prime}, \nu\right)$ for which $M_{c, \nu}(\mu)$ maps to $M_{c, \nu}(\tau)$ in degree $m$. These sets are either straight lines or finite sets.

The union of these sets is the reducibility locus of $M_{c, \nu}(\tau)$, denoted by $B_{\tau}$. Outside this reducibility locus $L_{c, \nu}(\tau)=M_{c, \nu}(\tau)$, and the character of this object is given by the formula

$$
\operatorname{ch} L(\tau)=\operatorname{ch} M(\tau)=\frac{t^{h_{c, \nu}(\tau)} X_{\tau}}{\sum_{n \geq 0}(-1)^{n} \Lambda^{n} \mathfrak{h}_{0} t^{n}} .
$$

This is the most generic case.

12.4.2. The next most generic case is a generic point on a line $\mathcal{L}_{\tau, \mu, m} \subset \mathcal{B}_{\mu, \tau}$.

Fix a pair $s, r$ such that $s, r \in \mathbb{Z}, r \neq 0, s \geq 0$, and consider a generic point $\left(c^{\prime}, \nu\right)$ on the line $c^{\prime}=\frac{\nu-s}{r}$.

Let $\tau$ be a Young diagram such that $s \in C_{\tau}$ (this exactly means that $M(\tau)$ is actually reducible at this point $(c, \nu))$, and denote $\mu:=\Gamma(\tau, s, \pm 1)$, where the sign equals $\operatorname{sign}(r)$. 
Then there are only two distinct Verma objects which map non-trivially into $M(\tau): M(\mu)$ and $M(\tau)$ itself (see Subsection [11.2). Both map uniquely (up to scalar multiple) into $M(\tau)$.

Lemma 12.4.1. The image of $M(\mu)$ in $M(\tau)$ is a simple $\underline{\mathcal{O}}_{c, \nu}$-object.

Proof. Assume $X_{\lambda}$ is a simple singular $\operatorname{Rep}\left(S_{\nu}\right)$-subobject in $\operatorname{Im}(M(\mu))$, and $X_{\lambda} \subset$ $M(\tau)$ lies in degree $m^{\prime}$. Then the action of $\boldsymbol{h}$ on $M(\tau)$ and $J(\tau) / \operatorname{Im}(M(\mu))$ gives (see Proposition 8.1.1):

$$
\begin{aligned}
& c^{\prime}=\frac{\nu-s}{r}, \\
& \frac{|\tau|^{2}-|\lambda|^{2}-(|\tau|-|\lambda|)}{2}+\operatorname{ct}(\tau)-c t(\lambda)=c^{\prime} m^{\prime}+(|\tau|-|\lambda|) \nu .
\end{aligned}
$$

By assumption, we obtain: $\mu=\lambda$, which implies $m=m^{\prime}$, and since $X_{\lambda}$ is a simple singular $\operatorname{Rep}\left(S_{\nu}\right)$-subobject in $\operatorname{Im}(M(\mu))$, this means that $X_{\lambda}$ coincides with the lowest weight in $\operatorname{Im}(M(\mu))$.

Thus $\operatorname{Im}(M(\mu))$ is simple.

We now want to show that there is a short exact sequence:

$$
0 \longrightarrow L(\mu) \longrightarrow M(\tau) \longrightarrow L(\tau) \longrightarrow 0 .
$$

This is equivalent to saying that $\operatorname{Im}(M(\mu))=J(\tau)$.

Lemma 12.4.2. $M(\tau) / \operatorname{Im}(M(\mu))$ is a simple $\underline{\mathcal{O}}_{c, \nu}$-object.

Proof. Assume $X_{\lambda}$ is a simple singular $\underline{\operatorname{Rep}}\left(S_{\nu}\right)$-subobject in $M(\tau) / \operatorname{Im}(M(\mu))$, and its preimage $X_{\lambda} \subset M(\tau)$ lies in degree $\overline{m^{\prime}}$.

Then the action of $\boldsymbol{h}$ on $M(\tau)$ and $J(\tau) / \operatorname{Im}(M(\mu))$ gives (see Proposition 8.1.1):

$$
\begin{aligned}
& c^{\prime}=\frac{\nu-s}{r} \\
& \frac{|\tau|^{2}-|\lambda|^{2}-(|\tau|-|\lambda|)}{2}+c t(\tau)-c t(\lambda)=c^{\prime} m^{\prime}+(|\tau|-|\lambda|) \nu .
\end{aligned}
$$

We now need to consider separately the case when $\lambda=\mu$. Indeed, in that case the above equations mean that $m^{\prime}=m$, and thus we have $X:=X_{\mu} \subset M(\tau)$ (not a singular subobject) which lies in degree $m^{\prime}$ and whose image in $M(\tau) / \operatorname{Im}(M(\mu))$ is not zero and singular.

The former implies that $y_{M(\tau)}\left(\mathfrak{h}_{0} \otimes X\right) \neq 0$ is a direct sum of simple $\operatorname{Rep}\left(S_{\nu}\right)$ objects lying in degree $m-1$ of $M(\tau)$.

The fact that image of $X$ in $M(\tau) / \operatorname{Im}(M(\mu))$ is singular would mean that

$$
y_{M(\tau)}\left(\mathfrak{h}_{0} \otimes X\right) \subset \operatorname{Im}(M(\mu)) .
$$

But this leads to a contradiction, since $\operatorname{Im}(M(\mu))$ lies in degrees strictly higher than $m-1$ of $M(\tau)$.

So $\lambda \neq \mu$, and this contradicts our assumption.

Thus for generic $\left(c^{\prime}, \nu\right)$ such that $c^{\prime}=\frac{\nu-s}{r}$, we have a short exact sequence:

$$
0 \longrightarrow L(\mu) \longrightarrow M(\tau) \longrightarrow L(\tau) \longrightarrow 0
$$


and a long exact sequence

$$
\begin{aligned}
& \cdots \rightarrow M(\Gamma(\tau, s, \pm l)) \rightarrow M(\Gamma(\tau, s, \pm(l-1))) \rightarrow \cdots \\
& \rightarrow M(\Gamma(\tau, s, \pm 1)) \rightarrow M(\tau) \rightarrow L(\tau) \rightarrow 0 .
\end{aligned}
$$

As before, the sign corresponds to the sign of $r$, and this sequence ends (on the left) with $M\left(\operatorname{rec}\left(0, \operatorname{core}_{(\nu-s)}(\tau)\right)\right)=M\left(\Gamma\left(\tau, s,-\widetilde{\tau_{j}}\right)\right)$ if $r<0$.

This allows us to compute the character of $L(\tau)$ by the Euler formula:

$$
\operatorname{ch} L(\tau)=\sum_{l \in \mathbb{Z}_{+}, \text {and } l \leq \check{\tau_{j_{s}}} \text { if } r<0}(-1)^{l} \operatorname{ch} M(\Gamma(\tau, s, \pm l)) .
$$

Now,

$$
\begin{aligned}
& h_{c, \nu}(\Gamma(\tau, s, \pm l))-h_{c, \nu}(\tau) \\
& =\sum_{1 \leq l^{\prime} \leq l} h_{c, \nu}\left(\Gamma\left(\tau, s, \pm l^{\prime}\right)\right)-h_{c, \nu}\left(\Gamma\left(\tau, s, \pm\left(l^{\prime}-1\right)\right)\right) \\
& =\sum_{1 \leq l^{\prime} \leq l} m_{l^{\prime}}=\sum_{1 \leq l^{\prime} \leq l} c(\nu-s)\left(\left|\Gamma\left(\tau, s, \pm l^{\prime}\right)\right|-\left|\Gamma\left(\tau, s, \pm\left(l^{\prime}-1\right)\right)\right|\right) \\
& =c(\nu-s)(|\Gamma(\tau, s, \pm l)|-|\tau|)=c(\nu-s)\left(j_{s}-k_{s, l} \pm l\right) .
\end{aligned}
$$

Here we denote by $m_{l^{\prime}}$ the degree of $M\left(\Gamma\left(\tau, s, \pm\left(l^{\prime}-1\right)\right)\right)$ in which lies the image of the lowest weight of $M\left(\Gamma\left(\tau, s, \pm l^{\prime}\right)\right)$, and use Lemma 10.0.20.

Using Subsection 12.2 and the fact that

$$
h_{c, \nu}(\Gamma(\tau, s, \pm l))-h_{c, \nu}(\tau)=c(\nu-s)\left(j_{s}-k_{s, l} \pm l\right)
$$

we obtain:

$$
\operatorname{ch} L(\tau)=\frac{t^{h_{c, \nu}(\tau)}\left(\sum_{l \in \mathbb{Z}_{+}, \text {and } l \leq \widetilde{\tau_{j_{s}}} \text { if } r<0}(-1)^{l} X_{\Gamma(\tau, s, \pm l)} t^{c(\nu-s)\left(j_{s}-k_{s, l} \pm l\right)}\right)}{\sum_{n \geq 0}(-1)^{n} \Lambda^{n} \mathfrak{h}_{0} t^{n}} .
$$

As before, the sign corresponds to the sign of $r$.

Example 12.4.3. Let $c \nu=1, \operatorname{Re}(c)>0$.

If $X_{\lambda}$ lies in degree $m^{\prime}$ of $M_{c, \nu}\left(\pi^{n}\right)$ as a singular $\underline{\operatorname{Rep}}\left(S_{\nu}\right)$-subobject $\left(\pi^{n}\right.$ is a column diagram with $n$ cells), then equation (8.2.1) gives us:

$$
m=(|\lambda|-n) c \nu-f(\lambda) c=(|\lambda|-n)-f(\lambda) c
$$

and Pieri's rule (Proposition 4.2.1) implies that $m \geq|| \lambda|-n|$. Since $m,|\lambda|, f(\lambda) \in$ $\mathbb{Z}_{+}$, we obtain that $c \in \mathbb{Q}_{>0}$.

We also have: $f(\lambda) \geq 0$, with equality iff $\lambda$ is a column diagram (see Lemma 8.2.1). So

$$
|| \lambda|-n| \leq m=(|\lambda|-n)-f(\lambda) c \leq|\lambda|-n
$$

which means that $f(\lambda)=0,|\lambda| \geq n$, i.e. $\lambda=\pi^{k}$ for some $k \geq n$ (in fact, $k>n$ ), and $X_{\pi^{k}}$ lies in degree $m=k-n$ of $M_{c, \nu}\left(\pi^{n}\right)$.

But by Pieri's rule (Proposition 4.2.1) , for $k>1, \mathfrak{h}_{0}^{\otimes k}$ contains only one copy of $X_{\pi^{k}} \cong \Lambda^{k} \mathfrak{h}_{0}$, and it does not lie inside $S^{k} \mathfrak{h}_{0}$. So $S^{k} \mathfrak{h}_{0}$ doesn't contain subobjects isomorphic to $X_{\pi^{k}}$.

So $M_{c, \nu}\left(\pi^{n}\right)$ has only one non-trivial singular $\operatorname{Rep}\left(S_{\nu}\right)$-subobject: $X_{\pi^{n+1}}$, which lies in degree 1 of $M_{c, \nu}\left(\pi^{n}\right)$. 
In this case, we can compute the character of $L_{c, \nu}\left(\pi^{n}\right)$ as in equation (12.4.2). We assumed that $c \nu=1$, so $h_{c, \nu}\left(\pi^{n}\right)=\frac{(\nu-1)(1-c \nu)}{2}+n=n$, hence

$$
\operatorname{ch} L_{c, \nu}\left(\pi^{n}\right)=\frac{\left(\sum_{l \in \mathbb{Z}_{+}}(-1)^{l} X_{\pi^{n+l}} t^{n+l}\right)}{\sum_{l \in \mathbb{Z}_{+}}(-1)^{l} \Lambda^{l} \mathfrak{h}_{0} t^{l}} .
$$

But $\Lambda^{n+l} \mathfrak{h}_{0} \cong X_{\pi^{n+l}}$ in $\underline{\operatorname{Rep}}\left(S_{\nu}\right)$, so

$$
\operatorname{ch} L_{c, \nu}\left(\pi^{n}\right)=\frac{\sum_{l \in \mathbb{Z}_{+}}(-1)^{l} \Lambda^{n+l} \mathfrak{h}_{0} t^{n+l}}{\sum_{l \in \mathbb{Z}_{+}}(-1)^{l} \Lambda^{l} \mathfrak{h}_{0} t^{l}}
$$

Note that $L_{c, \nu}(\emptyset) \cong X_{\emptyset}=\mathbb{1}$ as $\underline{\operatorname{Rep}}\left(S_{\nu}\right)$ (ind)-objects, with maps $x_{L_{c, \nu}(\emptyset)}$, $y_{L_{c, \nu}(\emptyset)}=0$.

Example 12.4.4. Similarly, for a generic point $(c, \nu)$ on the line $c \nu=k, k \in \mathbb{Z}_{>0}$, we have: $s=0, r=k$ and so

$$
\begin{gathered}
\Gamma\left(\pi^{n}, 0, \pm l\right)=X_{\pi^{n+l}}, \\
\operatorname{ch} L\left(\pi^{n}\right)=t^{k n-\frac{(\nu-1)(k-1)}{2}} \cdot \frac{\sum_{l \in \mathbb{Z}_{+}}(-1)^{l} \Lambda^{n+l} \mathfrak{h}_{0} t^{k l}}{\sum_{l \in \mathbb{Z}_{+}}(-1)^{l} \Lambda^{l} \mathfrak{h}_{0} t^{l}}
\end{gathered}
$$

and in particular,

$$
\operatorname{ch} L(\emptyset)=t^{-\frac{(\nu-1)(k-1)}{2}} \cdot \frac{\sum_{l \in \mathbb{Z}_{+}}(-1)^{l} \Lambda^{l} \mathfrak{h}_{0} t^{k l}}{\sum_{l \in \mathbb{Z}_{+}}(-1)^{l} \Lambda^{l} \mathfrak{h}_{0} t^{l}}=t^{-\frac{(\nu-1)(k-1)}{2}} \cdot \frac{\mathbf{c h}_{t} S \mathfrak{h}_{0}}{\mathbf{c h}_{t^{k}} S \mathfrak{h}_{0}}
$$

(see [3] or [10, Corollary 3.50] for the corresponding result for $\left.\mathcal{O}\left(H_{c}(n)\right)\right)$.

As in the case when $k=1$, we can also compute the character of $L_{c, \nu}(\emptyset)$ explicitly:

Proposition 12.4.5. Let $(c, \nu)$ be a generic point on the line $c \nu=k$, where $k \in \mathbb{Z}_{>0}$ is fixed. Then

$$
c h_{q} \operatorname{Hom}_{S_{\nu}}\left(X_{\mu}, L_{c, \nu}(\emptyset)\right)=\frac{q^{|\mu|} s_{\mu}\left(1, q, q^{2}, \ldots, q^{k-2}\right)}{\prod_{2 \leq j \leq k}\left(1-q^{j}\right)} .
$$

Proof. From the exact sequence

$$
\ldots \longrightarrow M\left(\pi^{l}\right) \longrightarrow M\left(\pi^{l-1}\right) \longrightarrow \ldots \longrightarrow M\left(\pi^{1}\right) \longrightarrow M(\emptyset) \longrightarrow L(\emptyset) \longrightarrow 0
$$

and the character formula Corollary 12.3 .3 for $\operatorname{Hom}_{\underline{\operatorname{Rep}\left(S_{\nu}\right)}}\left(X_{\mu}, S \mathfrak{h}_{0} \otimes X_{\pi^{l}}\right)$, we have:

$$
c h_{q} \operatorname{Hom}_{S_{\nu}}\left(X_{\mu}, L(\emptyset)\right)=(1-q) \sum_{l \geq 0} \sum_{\lambda \in \mathcal{P}}(-1)^{l} q^{k l} \bar{\gamma}_{\Lambda^{l} \mathfrak{h}_{0}, \mu}^{\lambda} \frac{q^{|\lambda|} s_{\lambda}\left(1, q, q^{2}, \ldots\right)}{\prod_{j \geq 1}\left(1-q^{j}\right)} .
$$

One immediately sees that this is the $q$-adic limit, as $n$ tends to infinity, of the sequence of formal power series

$$
P_{n}(q):=(1-q) \sum_{l \geq 0} \sum_{\lambda \vdash n}(-1)^{l} q^{k l} \gamma_{\Lambda^{l} \mathfrak{h}_{0}, \tilde{\mu}(n)}^{\lambda} s_{\lambda}\left(1, q, q^{2}, \ldots\right)
$$

where $\mathfrak{h}_{0}$ is the reflection representation of $S_{n}$.

We now give a formula for $P_{n}(q)$. First, recall that (see [18, Chapter I, Par. 7])

$$
\gamma_{\Lambda^{l} \mathfrak{h}_{0}, \tilde{\mu}(n)}^{\lambda}=\frac{1}{n !} \sum_{w \in S_{n}} \chi^{\Lambda^{l} \mathfrak{h}_{0}}(w) \chi^{\tilde{\mu}(n)}(w) \chi^{\lambda}(w)
$$


(here $\chi^{\beta}(w)=\left.\operatorname{tr}\right|_{\beta}(w)$ is the value at $w$ of the character of $S_{n}$ corresponding to the irreducible representation $\beta$ ).

Now, from the exact sequence

$0 \rightarrow S \mathfrak{h}_{0} \otimes \Lambda^{n-1} \mathfrak{h}_{0} \longrightarrow \cdots \longrightarrow S \mathfrak{h}_{0} \otimes \Lambda^{m} \mathfrak{h}_{0} \longrightarrow \cdots \longrightarrow S \mathfrak{h}_{0} \otimes \mathfrak{h}_{0} \longrightarrow S \mathfrak{h}_{0} \longrightarrow \mathbb{C} \rightarrow 0$ we have:

$$
\begin{aligned}
& \sum_{l \geq 0}(-1)^{l} q^{k l} \chi^{\Lambda^{l} \mathfrak{h}_{0}}(w)=\frac{1}{\left.\sum_{l \geq 0} q^{k l} \operatorname{tr}\right|_{S^{l} \mathfrak{h}_{0}}(w)} \\
& =\frac{1}{\left.\sum_{l \geq 0} q^{k l}\left(1-q^{k}\right) \operatorname{tr}\right|_{S^{l} \mathfrak{h}}(w)}=\frac{1}{1-q^{k}} \prod_{1 \leq j \leq \ell(\rho(w))}\left(1-q^{k \rho(w)_{j}}\right)
\end{aligned}
$$

(here $\rho(w)$ is the cycle type of $w$ ).

On the other hand, we have (see e.g. [18, Par. 1, (7.7), (7.8), p. 114]):

$$
\sum_{\lambda \vdash n} \chi^{\lambda}(w) s_{\lambda}\left(1, q, q^{2}, \ldots\right)=\prod_{1 \leq j \leq \ell(\rho(w))} \frac{1}{1-q^{\rho(w)_{j}}} .
$$

Thus we obtain:

$$
\begin{aligned}
& P_{n}(q)=(1-q) \sum_{l \geq 0} \sum_{\lambda \vdash n}(-1)^{l} q^{k l} \gamma_{\Lambda^{l} \mathfrak{h}_{0}, \tilde{\mu}(n)}^{\lambda} s_{\lambda}\left(1, q, q^{2}, \ldots\right) \\
& =\frac{1-q}{1-q^{k}} \frac{1}{n !} \sum_{w \in S_{n}} \chi^{\tilde{\mu}(n)}(w) \prod_{1 \leq j \leq \ell(\rho(w))} \frac{1-q^{k \rho(w)_{j}}}{1-q^{\rho(w)_{j}}} \\
& =\frac{1-q}{1-q^{k}} \frac{1}{n !} \sum_{w \in S_{n}} \chi^{\tilde{\mu}(n)}(w) \prod_{1 \leq j \leq \ell(\rho(w))}\left(1+q^{\rho(w)_{j}}+q^{2 \rho(w)_{j}}+\cdots+q^{k \rho(w)_{j}}\right) \\
& =\frac{1-q}{1-q^{k}} s_{\tilde{\mu}(n)}\left(1, q, \ldots, q^{k-1}\right)
\end{aligned}
$$

Taking the $q$-adic limit when $n \rightarrow \infty$, we obtain: the $q$-adic limit of $s_{\tilde{\mu}(n)}(1, q, \ldots$, $\left.q^{k-1}\right)$ is $\frac{q^{|\mu|} s_{\mu}\left(1, q, q^{2}, \ldots, q^{k-2}\right)}{\prod_{1 \leq j \leq k-1}\left(1-q^{j}\right)}$, and thus

$$
c h_{q} \operatorname{Hom}_{S_{\nu}}\left(X_{\mu}, L(\emptyset)\right)=\frac{q^{|\mu|} s_{\mu}\left(1, q, q^{2}, \ldots, q^{k-2}\right)}{\prod_{2 \leq j \leq k}\left(1-q^{j}\right)} .
$$

Remark 12.4.6. Note that $s_{\mu}\left(1, q, q^{2}, \ldots, q^{k-2}\right)=0$ if $l(\mu) \geq k$, so $\operatorname{Hom}_{S_{\nu}}\left(X_{\mu}, L(\emptyset)\right)$ $=0$ whenever $l(\mu) \geq k$.

\section{Length of Verma obJeCts}

In this section, we will discuss the set of points $(c, \nu)$ such that the Verma object $M_{c, \nu}(\tau)$ is of finite length. We will prove the following theorem:

Theorem 13.0.7. For any $c \notin \mathbb{Q}<0$ and any Young diagram $\tau$, the Verma object $M_{c, \nu}(\tau)$ is of finite length.

By Subsection 11.1 $M_{c, \nu}(\tau)$ has infinite length whenever $(c, \nu)$ is the intersection point of infinitely many curves of form $\frac{1}{c}=\frac{\nu-s}{r}$ where $s \in C_{\tau}, r \in \mathbb{Z} \backslash\{0\}$.

For instance, when $\tau=\emptyset$, we have:

Lemma 13.0.8. For any $c \in \mathbb{Q}<0$ there exists $\nu \in \mathbb{Q}$ such that the Verma object $M_{c, \nu}(\emptyset)$ has infinite length. 
Proof. Indeed, for any $c \in \mathbb{Q}_{<0}$ and any $\nu \in \mathbb{Q}$ such that $\operatorname{den}(\nu)|\operatorname{num}(c), \operatorname{den}(c)|$ $(\operatorname{num}(\nu)+\operatorname{den}(\nu))$ (i.e. $\left.\operatorname{num}(\nu) \operatorname{den}(\nu)^{-1} \equiv-1 \bmod \operatorname{den}(c)\right)$, we have:

$$
\left\{s \in \mathbb{Z}_{+} \mid c(\nu-s) \in \mathbb{Z}_{>0}\right\}=\left\{s \in \mathbb{Z}_{+} \mid s \equiv-1 \quad \bmod \operatorname{den}(c), s>\nu\right\} .
$$

Now, recall that $C_{\emptyset}=\mathbb{Z}_{+}$. So any $s$ such that $s \equiv-1 \bmod \operatorname{den}(c), s>\nu$ gives us a non-trivial morphism $M_{c, \nu}\left(\tau^{s}\right) \longrightarrow M_{c, \nu}(\emptyset)$. The image of each of these morphisms has a simple object $L_{c, \nu}\left(\tau^{s}\right)$ as a quotient, and since all the weights $\tau^{s}$ are different, each of these simple objects $L_{c, \nu}\left(\tau^{s}\right)$ contributes to the length of $M_{c, \nu}(\emptyset)$. This proves that $M_{c, \nu}(\emptyset)$ has infinite length.

The proof consists of two parts: proving that whenever $c \in \mathbb{R}_{>0}$, the Verma object $M_{c, \nu}(\tau)$ is of finite length (we will prove this statement in Subsection 13.2), and proving that whenever $c \notin \mathbb{Q}$, the Verma object $M_{c, \nu}(\tau)$ is of finite length, too (we will prove that in Subsection 13.3.

Remark 13.0.9. In the classical case, all modules in $\mathcal{O}\left(H_{c}(n)\right)$ have finite length. See [10, Corollary 3.26].

13.1. Bounds on the graded space $\operatorname{Hom}_{\operatorname{Rep}\left(S_{\nu}\right)}\left(X_{\mu}, S \mathfrak{h}_{0} \otimes X_{\tau}\right)$. In this subsection, we give a bound on the least degree in which a simple $\operatorname{Rep}\left(S_{\nu}\right)$ object $X_{\mu}$ can lie in the $\operatorname{Rep}\left(S_{\nu}\right)$ ind-object $S \mathfrak{h}_{0} \otimes X_{\tau}$.

Recall that we have the following corollary of Lemma 12.3.1

Corollary 13.1.1. For $\tau=\emptyset$, we have:

$$
c h_{q}\left(\operatorname{Hom}_{S_{n}}\left(\tilde{\mu}(n), \mathbb{C}\left[x_{1}, \ldots, x_{n}\right]\right)\right)=s_{\tilde{\mu}(n)}\left(1, q, q^{2}, \ldots\right) .
$$

By the definition of a Schur symmetric function, $s_{\tilde{\mu}(n)}\left(1, q, q^{2}, \ldots\right)$ is divisible by $q^{\sum_{k} \mu_{k} k}$. So for any $n>>0$, the minimal degree of $S \mathfrak{h}=\mathbb{C}\left[x_{1}, \ldots, x_{n}\right]$ in which $\tilde{\mu}(n)$ appears is greater than or equal to $\sum_{k} \mu_{k} k$. By the description of Deligne's category $\underline{\operatorname{Rep}}\left(S_{\nu}\right)$, this implies:

Corollary 13.1.2. For any $\nu$, we have: the minimal degree of $S \mathfrak{h}$ (and thus of $S \mathfrak{h}_{0}$ as well) in which $X_{\mu}$ appears is greater than or equal to $\sum_{k} \mu_{k} k$.

For the convenience of the reader, we will use the following notation:

Notation 13.1.3. For a Young diagram $\mu$, the sum $\sum_{k} \mu_{k} k$ will be denoted by $n(\mu)$ (cf. [18]).

The function $n(\cdot): \mathcal{P} \rightarrow \mathbb{Z}_{+}$has the following trivial property (see also 18, Chapter I]):

Lemma 13.1.4. For any Young diagram $\mu$, we have:

$$
|\mu| \leq n(\mu) \leq \frac{|\mu|(|\mu|+1)}{2} .
$$

Proof. It is easy to see that when we consider the function $n(\cdot)$ on the set of Young diagrams of fixed size $k$, the maximum is obtained for the Young diagram $\pi^{k}$ (a column of length $k$ ), and it is $n\left(\pi^{k}\right)=\frac{|k|(|k|+1)}{2}$, while the minimum is obtained for $\tau^{k-1}$ (a row of length $k$ ), and it is $n\left(\tau^{k-1}\right)^{2}=k$.

We now generalize this result to the following: 
Proposition 13.1.5. Consider the $\mathbb{Z}_{+}$-graded complex vector space $\operatorname{Hom}_{\operatorname{Rep}\left(S_{\nu}\right)}\left(X_{\mu}\right.$, $S \mathfrak{h}_{0} \otimes X_{\tau}$ ) (the grading inherited from $\left.S \mathfrak{h}_{0}\right)$. Let $m$ be a grade of this space containing a non-zero morphism. Then

$$
m \geq n(\mu)-\ell(\mu)|\tau|-\frac{|\tau|^{2}}{2}-\frac{|\tau|}{2} .
$$

Proof. Recall that any object of $\operatorname{Rep}\left(S_{\nu}\right)$ is isomorphic to its dual, so

$$
\operatorname{Hom}_{\underline{\operatorname{Rep}}\left(S_{\nu}\right)}\left(X_{\mu}, S \mathfrak{h}_{0} \otimes X_{\tau}\right) \cong \operatorname{Hom}_{\underline{\operatorname{Rep}}\left(S_{\nu}\right)}\left(X_{\mu} \otimes X_{\tau}, S \mathfrak{h}_{0}\right)
$$

as $\mathbb{Z}_{+}$-graded vector spaces.

Assume $m \geq 0$ is such that $\operatorname{Hom}_{\operatorname{Rep}\left(S_{\nu}\right)}\left(X_{\mu} \otimes X_{\tau}, S \mathfrak{h}_{0}\right) \neq 0$. This means that $X_{\mu} \otimes X_{\tau}$ and $S^{m} \mathfrak{h}_{0}$ have a common composition factor. Now, $X_{\tau} \subset \mathfrak{h}_{0}^{\otimes|\tau|}$, so $X_{\mu} \otimes \mathfrak{h}_{0}^{\otimes|\tau|}$ and $S^{m} \mathfrak{h}_{0}$ have a common composition factor.

A simple subobject $X_{\lambda}$ of $X_{\mu} \otimes \mathfrak{h}_{0}^{\otimes|\tau|}$ satisfies the condition arising from Pieri's rule (Proposition 4.2.1):

Condition 13.1.6. The Young diagram $\lambda$ can be obtained from $\mu$ by performing at most $|\tau|$ steps, each consisting of either adding a cell, deleting a cell or moving a cell (i.e. deleting a cell and then adding a cell).

We know that for some Young diagram $\lambda$ satisfying Condition 13.1.6, we have: $X_{\lambda} \subset S^{m} \mathfrak{h}_{0}$. Corollary 13.1 .2 then tells us that

$$
m \geq \sum_{k} \lambda_{k} k
$$

We are left with the following problem:

For two Young diagrams $\mu, \lambda$ satisfying Condition 13.1.6, find an upper bound for $n(\mu)-n(\lambda)$ in terms of $|\tau|, \ell(\mu)$.

By the Condition 13.1.6, the difference $n(\mu)-n(\lambda)$ is maximal when $\lambda$ is obtained from $\mu$ by removing a sequence of $|\tau|$ boxes starting with bottom row and progressing upwards, from right to left. Namely, the maximum of the difference is obtained for $\lambda:=\lambda^{0}$, where $\lambda^{0}$ is constructed as follows:

Let $k_{0} \geq 0$ be such that $\sum_{i \geq k_{0}+1} \mu_{i} \leq|\tau|<\sum_{i \geq k_{0}} \mu_{i}$. Then $\lambda^{0}$ is a Young diagram of length $k_{0}$, defined as

$$
\lambda_{i}^{0}:= \begin{cases}\mu_{i} & \text { if } 1 \leq i \leq k_{0}-1, \\ \mu_{k_{0}}-\left(|\tau|-\sum_{i \geq k+1} \mu_{i}\right) & \text { if } i=k_{0} .\end{cases}
$$

Example 13.1.7. Let $|\tau|=6, \mu=(5,4,4,3,2)$. Then $k_{0}=3$, and $\lambda^{0}=(5,4,3)$ :

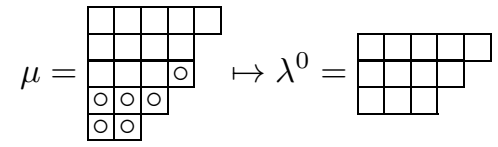

We now give an upper bound for $n(\mu)-n\left(\lambda^{0}\right)$. Writing it out explicitly, we obtain

$$
\begin{aligned}
& n(\mu)-n\left(\lambda^{0}\right)=\sum_{k \geq k_{0}+1} \mu_{k} k+k_{0}\left(|\tau|-\sum_{i \geq k+1} \mu_{i}\right) \\
& =\sum_{k \geq k_{0}+1} \mu_{k}\left(k-k_{0}\right)+k_{0}|\tau|=n\left(\bar{\mu}^{\left(k_{0}\right)}\right)+k_{0}|\tau| .
\end{aligned}
$$


where $\bar{\mu}^{(k)}:=\left(\mu_{k_{0}+1}, \mu_{k_{0}+2}, \ldots\right)$ (the Young diagram obtained by removing the first $k_{0}$ rows of $\mu$ ). We know that $\left|\bar{\mu}^{(k)}\right| \leq|\tau|$ and $k_{0} \leq \ell(\mu)$, so by Lemma 13.1.4. we have:

$$
n\left(\bar{\mu}^{\left(k_{0}\right)}\right)+k_{0}|\tau| \leq \frac{|\tau|(|\tau|+1)}{2}+\ell(\mu)|\tau|
$$

Thus for any two Young diagrams $\mu, \lambda$ satisfying Condition 13.1.6 we have:

$$
n(\mu)-n(\lambda) \leq l(\mu)|\tau|+\frac{|\tau|(|\tau|+1)}{2}
$$

which implies

$$
m \geq n(\lambda) \geq n(\mu)-l(\mu)|\tau|-\frac{|\tau|(|\tau|+1)}{2} .
$$

13.2. Length of Verma objects for $c \in \mathbb{R}_{>0}$.

Proposition 13.2.1. For any $c \in \mathbb{R}_{>0}$, the Verma object $M_{c, \nu}(\tau)$ is of finite length.

Proof. Fix $c, \nu$ such that $c \in \mathbb{R}_{>0}$. We need to prove that $M(\tau)$ has only finitely many composition factors. Let $\left\{L\left(\mu^{j}\right)\right\}_{j \in J}$ be the composition factors of $M(\tau)$.

Since $L\left(\mu^{j}\right)$ is a composition factor of $M(\tau)$, there exists a positive integer $m^{j}$ such that $h_{c, \nu}\left(\mu^{j}\right)=h_{c, \nu}(\tau)+m^{j}$. By Proposition 8.1.1, to prove that $J$ is a finite set, it is enough to prove that there exist only finitely many Young diagrams $\mu$ such that $X_{\mu}$ lies in degree $m$ of $M(\tau)$ and

$$
m=c \nu(|\mu|-|\tau|)+c\left(\frac{|\tau|^{2}-|\mu|^{2}-(|\tau|-|\mu|)}{2}+c t(\tau)-c t(\mu)\right) .
$$

By Proposition 13.1.5, it is, in fact, enough to prove that

$$
\begin{aligned}
& c \nu(|\mu|-|\tau|)+c\left(\frac{|\tau|^{2}-|\mu|^{2}-(|\tau|-|\mu|)}{2}+c t(\tau)-c t(\mu)\right) \\
& <n(\mu)-\ell(\mu)|\tau|-\frac{|\tau|^{2}}{2}-\frac{|\tau|}{2} .
\end{aligned}
$$

for all but finitely many Young diagrams $\mu$.

Recall that $\operatorname{ct}(\mu)=n(\mu)-n(\mu)$, so subtracting LHS from RHS in the above expression, we obtain

$$
\begin{aligned}
& -c \nu|\mu|+c \nu|\tau|-c f(\tau)+c \frac{|\mu|^{2}-|\mu|}{2}+c \cdot n(\mu)-c \cdot n(\mu) \\
& +n(\mu)-\ell(\mu)|\tau|-\frac{|\tau|^{2}}{2}-\frac{|\tau|}{2}=c \frac{|\mu|^{2}-|\mu|}{2} \\
& -c \nu|\mu|+c \cdot n(\mu)-(c-1) \cdot n(\mu)-\ell(\mu)|\tau|-\frac{|\tau|^{2}}{2}-\frac{|\tau|}{2}+c \nu|\tau|-c f(\tau)
\end{aligned}
$$

where $f(\tau):=\frac{|\tau|^{2}-|\tau|}{2}+\operatorname{ct}(\tau)$.

We need to show that for all but finitely many Young diagrams $\mu$, the expression below

$c \frac{|\mu|^{2}-|\mu|}{2}-c \nu|\mu|+c \cdot n(\mu)-(c-1) \cdot n(\mu)-\ell(\mu)|\tau|-\frac{|\tau|^{2}}{2}-\frac{|\tau|}{2}+c \nu|\tau|-c f(\tau)$

is positive (keep in mind that the parameter $\nu$ and the Young diagram $\tau$ remain fixed). 
Since $l(\mu) \leq|\mu|$, it is in fact enough to check that for all Young diagrams $\mu$, the expression

$$
\frac{c}{2}|\mu|^{2}-\left(\frac{c}{2}+c \nu+|\tau|\right)|\mu|+c \cdot n(\mu \breve{)}-(c-1) \cdot n(\mu)
$$

is bounded below by a polynomial in $|\mu|$ of positive degree, and with a positive leading coefficient.

We now have to consider two cases separately:

- If $0<c<1$, then $c \cdot n(\mu)-(c-1) \cdot n(\mu)>0$, and so the expression in equation (13.2.1) is bounded below by the polynomial

$$
\frac{c}{2}|\mu|^{2}-\left(\frac{c}{2}+c \nu+|\tau|\right)|\mu| .
$$

- If $c \geq 1$, then using Lemma 13.1.4, we obtain:

$$
\begin{aligned}
& \frac{c}{2}|\mu|^{2}-\left(\frac{c}{2}+c \nu+|\tau|\right)|\mu|+c \cdot n(\mu \breve{l})-(c-1) \cdot n(\mu) \\
& \geq \frac{c}{2}|\mu|^{2}-\left(\frac{c}{2}+c \nu+|\tau|\right)|\mu|+c|\mu|-(c-1) \frac{|\mu|(|\mu|+1)}{2} \\
& =\frac{1}{2}|\mu|^{2}+\left(\frac{1}{2}-c \nu-|\tau|\right)|\mu| .
\end{aligned}
$$

We conclude that when $c \geq 1$, the expression in equation (13.2.1) is bounded below by the polynomial

$$
\frac{1}{2}|\mu|^{2}+\left(\frac{1}{2}-c \nu-|\tau|\right)|\mu|
$$

as wanted.

\subsection{Length of Verma objects for $c \notin \mathbb{Q}$.}

Proposition 13.3.1. For any $c \notin \mathbb{Q}$, the Verma object $M_{c, \nu}(\tau)$ is of finite length.

Proof. Assume that for some $c \notin \mathbb{Q}, \nu$, a Verma object $M_{c, \nu}(\tau)$ has infinite length.

Let $\left\{L_{c, \nu}\left(\mu^{j}\right)\right\}_{j \in J}$ be the composition factors of $M_{c, \nu}(\tau)$.

Since $L_{c, \nu}\left(\mu^{j}\right)$ is a composition factor of $M_{c, \nu}(\tau)$, there exists a positive integer $m^{j}$ such that $h_{c, \nu}\left(\mu^{j}\right)=h_{c, \nu}(\tau)+m^{j}$, and $X_{\mu^{j}} \subset S^{m^{j}} \mathfrak{h}_{0} \otimes \tau$. Similarly to the proof of Proposition 8.1.1, for any $j$ we have:

$$
\left(c^{\prime}, \nu\right) \in \mathcal{L}_{\tau, \mu^{j}, m^{j}} .
$$

Since $\left(c^{\prime}, \nu\right) \in \mathcal{L}_{\tau, \mu^{j}, m^{j}}$ for any $j$, and $c \notin \mathbb{Q}$, Subsection 11.2 implies that for all $j$ the lines $\mathcal{L}_{\tau, \mu^{j}, m^{j}}$ coincide; that is, the equations

$$
\frac{1}{c} m^{j}+\nu\left(|\tau|-\left|\mu^{j}\right|\right)=\frac{|\tau|^{2}-\left|\mu^{j}\right|^{2}-\left(|\tau|-\left|\mu^{j}\right|\right)}{2}+\operatorname{ct}(\tau)-\operatorname{ct}\left(\mu^{j}\right)
$$

define the same line for all $j$.

This means that there exist constants $C, C^{\prime} \in \mathbb{Q}$ such that for any $j$,

$$
\begin{aligned}
& \frac{1}{\left|\mu^{j}\right|-|\tau|}\left(\frac{|\tau|^{2}-\left|\mu^{j}\right|^{2}-\left(|\tau|-\left|\mu^{j}\right|\right)}{2}+\operatorname{ct}(\tau)-\operatorname{ct}\left(\mu^{j}\right)\right)=C^{\prime} \\
& \text { and } \frac{\left|\mu^{j}\right|-|\tau|}{m^{j}}=C \text {. }
\end{aligned}
$$


Since $X_{\mu^{j}} \subset S^{m^{j}} \mathfrak{h}_{0} \otimes \tau$, Pieri's rule (Proposition 4.2.1) implies that $|C| \leq 1$, and Proposition 13.1.5 implies that for any $j$,

$$
m^{j} \geq \sum_{k \geq 1}\left(\mu^{j}\right)_{k} k-\ell\left(\mu^{j}\right)|\tau|-\frac{|\tau|^{2}}{2}-\frac{|\tau|}{2} .
$$

So there exist constants $C, C^{\prime}$ such that for any $j$, the following conditions hold for $\mu:=\mu^{j}, m:=m^{j}$ :

\section{Condition 13.3.2.}

$$
\begin{aligned}
& \frac{1}{|\mu|-|\tau|}\left(\frac{|\mu|^{2}-|\mu|}{2}+\operatorname{ct}(\mu)-f(\tau)\right)=C^{\prime}, \\
& \frac{|\mu|-|\tau|}{m}=C, \\
& m^{j} \geq \sum_{k \geq 1}(\mu)_{k} k-\ell(\mu)|\tau|-\frac{|\tau|^{2}}{2}-\frac{|\tau|}{2} .
\end{aligned}
$$

with $f(\tau)=\frac{|\tau|^{2}-|\tau|}{2}+\operatorname{ct}(\tau)$ as in Subsection 8.2 .

We will show that for fixed $C, C^{\prime}$ as above, Condition 13.3 .2 can only hold for a finite number of Young diagrams $\mu$.

First, notice that we can assume that $C>0$ (otherwise $\mu \leq|\tau|$ and we are done).

Combining the second and third requirements of Condition 13.3 .2 gives:

$$
C^{-1}(|\mu|-|\tau|) \geq n(\mu)-\ell(\mu)|\tau|-\frac{|\tau|^{2}}{2}-\frac{|\tau|}{2} .
$$

i.e.

$$
n(\mu) \leq \frac{|\tau|^{2}}{2}+\frac{|\tau|}{2}-C^{-1}|\tau|+\ell(\mu)|\tau|+C^{-1}|\mu| .
$$

The first requirement of Condition 13.3 .2 gives:

$$
\frac{|\mu|^{2}-|\mu|}{2}+c t(\mu)-C^{\prime}|\mu|=f(\tau)-C^{\prime}|\tau| \text {. }
$$

Now, $\operatorname{ct}(\mu)=n(\mu)-n(\mu)$, so combining inequality (13.3.4) and equation (13.3.5) gives:

$$
\begin{aligned}
& \frac{|\mu|^{2}-|\mu|}{2}-C^{\prime}|\mu|=f(\tau)-C^{\prime}|\tau|-c t(\mu)=f(\tau)-C^{\prime}|\tau|+n(\mu)-n(\mu) \\
& \leq f(\tau)-C^{\prime}|\tau|+\frac{|\tau|^{2}}{2}+\frac{|\tau|}{2}-C^{-1}|\tau|+\ell(\mu)|\tau|+C^{-1}|\mu|-n(\mu) .
\end{aligned}
$$

The last inequality means that for any Young diagram $\mu$ satisfying Condition 13.3.2. the expression

$$
\frac{|\mu|^{2}}{2}-\left(\frac{1}{2}+C^{\prime}+C^{-1}\right)|\mu|-l(\mu)|\tau|+n(\mu)
$$

is bounded by a function of $\tau$ (which is fixed).

We now consider the expression $n(\mu \breve{)}$. We can rewrite it as

$$
n(\mu)=\sum_{1 \leq k \leq \ell(\mu)}\left(\check{\mu_{k}}-1\right)(k-1)+\frac{l(\mu)^{2}}{2}-\frac{l(\mu)}{2}+|\mu| .
$$


The summand $\sum_{1 \leq k \leq \ell(\mu)}\left(\check{\mu_{k}}-1\right)(k-1)$ is clearly non-negative, so

$$
n(\mu) \geq \frac{l(\mu)^{2}}{2}-\frac{l(\mu)}{2}+|\mu| .
$$

Applying the inequality $n(\mu)^{\dagger} \geq \frac{l(\mu)^{2}}{2}-\frac{l(\mu)}{2}+|\mu|$, to inequality (13.3.6), we see that for any Young diagram $\mu$ satisfying Condition 13.3.2, the expression

$$
\frac{|\mu|^{2}}{2}+\left(\frac{1}{2}-C^{\prime}-C^{-1}\right)|\mu|+\frac{l(\mu)^{2}}{2}-\left(\frac{1}{2}+|\tau|\right) l(\mu)
$$

is bounded by a function of $\tau$. This clearly means that in such a case, $|\mu|$ is bounded (by a function of $\tau$ ); we conclude that $M_{c, \nu}(\tau)$ has finitely many composition factors.

\section{ACKNOWLEDGMENTS}

I would like to thank my advisor, Pavel Etingof, for suggesting the problem and guiding me throughout the project. I would also like to thank Alexander Kleshchev for an explanation on the blocks of the category $\mathcal{O}\left(H_{c}(n)\right)$, and Avraham Aizenbud for the helpful discussions.

I am very grateful to Oleksandr Tsymbaliuk for his comments on the paper, and to the anonymous referee for the helpful comments and suggestions.

\section{REFERENCES}

[1] Roman Bezrukavnikov and Pavel Etingof, Parabolic induction and restriction functors for rational Cherednik algebras, Selecta Math. (N.S.) 14 (2009), no. 3-4, 397-425, DOI 10.1007/s00029-009-0507-z. MR2511190 (2010e:20007)

[2] Yuri Berest, Pavel Etingof, and Victor Ginzburg, Cherednik algebras and differential operators on quasi-invariants, Duke Math. J. 118 (2003), no. 2, 279-337, DOI 10.1215/S0012-7094-0311824-4. MR1980996 (2004f:16039)

[3] Yuri Berest, Pavel Etingof, and Victor Ginzburg, Finite-dimensional representations of rational Cherednik algebras, Int. Math. Res. Not. 19 (2003), 1053-1088, DOI 10.1155/S1073792803210205. MR1961261 (2004h:16027)

[4] Emmanuel Briand, Rosa Orellana, and Mercedes Rosas, The stability of the Kronecker product of Schur functions (English, with English, French and Spanish summaries), 22nd International Conference on Formal Power Series and Algebraic Combinatorics (FPSAC 2010), Discrete Math. Theor. Comput. Sci. Proc., AN, Assoc. Discrete Math. Theor. Comput. Sci., Nancy, 2010, pp. 557-567. MR2673866 (2012m:05404)

[5] Jonathan Comes and Victor Ostrik, On blocks of Deligne's category $\operatorname{Rep}\left(S_{t}\right)$, Adv. Math. 226 (2011), no. 2, 1331-1377, DOI 10.1016/j.aim.2010.08.010. MR2737787 (2012b:20020)

[6] P. Deligne, La catégorie des représentations du groupe symétrique $S_{t}$, lorsque $t$ n'est pas un entier naturel (French, with English and French summaries), Algebraic groups and homogeneous spaces, Tata Inst. Fund. Res. Stud. Math., Tata Inst. Fund. Res., Mumbai, 2007, pp. 209-273. MR2348906 (2009b:20021)

[7] Richard Dipper and Gordon James, Blocks and idempotents of Hecke algebras of general linear groups, Proc. London Math. Soc. (3) 54 (1987), no. 1, 57-82, DOI 10.1112/plms/s354.1.57. MR872250 (88m:20084)

[8] Karin Erdmann and Daniel K. Nakano, Representation type of Hecke algebras of type A, Trans. Amer. Math. Soc. 354 (2002), no. 1, 275-285 (electronic), DOI 10.1090/S0002-994701-02848-3. MR.1859276 (2002j:20011)

[9] Pavel Etingof, Representation theory in complex rank, I, Transform. Groups 19 (2014), no. 2, 359-381, DOI 10.1007/s00031-014-9260-2. MR3200430

[10] P. Etingof, X. Ma, Lecture notes on Cherednik algebras; arXiv:1001.0432v4 [math.RT]. 
[11] Pavel Etingof and Emanuel Stoica, Unitary representations of rational Cherednik algebras, Represent. Theory 13 (2009), 349-370, DOI 10.1090/S1088-4165-09-00356-2. With an appendix by Stephen Griffeth. MR2534594 (2011c:16081)

[12] William Fulton and Joe Harris, Representation theory: A first course; Readings in Mathematics, Graduate Texts in Mathematics, vol. 129, Springer-Verlag, New York, 1991. MR.1153249 (93a:20069)

[13] Victor Ginzburg, Nicolas Guay, Eric Opdam, and Raphaël Rouquier, On the category $\mathcal{O}$ for rational Cherednik algebras, Invent. Math. 154 (2003), no. 3, 617-651, DOI 10.1007/s00222003-0313-8. MR2018786 (2005f:20010)

[14] Eugene Gorsky, Alexei Oblomkov, Jacob Rasmussen, and Vivek Shende, Torus knots and the rational DAHA, Duke Math. J. 163 (2014), no. 14, 2709-2794, DOI 10.1215/001270942827126. MR 3273582

[15] I. Gordon and J. T. Stafford, Rational Cherednik algebras and Hilbert schemes, Adv. Math. 198 (2005), no. 1, 222-274, DOI 10.1016/j.aim.2004.12.005. MR2183255 (2008i:14006)

[16] Friedrich Knop, A construction of semisimple tensor categories (English, with English and French summaries), C. R. Math. Acad. Sci. Paris 343 (2006), no. 1, 15-18, DOI 10.1016/j.crma.2006.05.009. MR2241951(2007b:18008)

[17] I. Losev, Towards multiplicities for categories $O$ of cyclotomic rational Cherednik algebras, arXiv:1207.1299v2 [math.RT].

[18] I. G. Macdonald, Symmetric functions and Hall polynomials, 2nd ed., Oxford Mathematical Monographs, The Clarendon Press, Oxford University Press, New York, 1995. With contributions by A. Zelevinsky; Oxford Science Publications. MR.1354144 (96h:05207)

[19] Andrew Mathas, Iwahori-Hecke algebras and Schur algebras of the symmetric group, University Lecture Series, vol. 15, American Mathematical Society, Providence, RI, 1999. MR.1711316 (2001g:20006)

[20] Akhil Mathew, Categories parametrized by schemes and representation theory in complex rank, J. Algebra 381 (2013), 140-163, DOI 10.1016/j.jalgebra.2013.01.029. MR3030515

[21] Raphaël Rouquier, $q$-Schur algebras and complex reflection groups (English, with English and Russian summaries), Mosc. Math. J. 8 (2008), no. 1, 119-158, 184. MR2422270(2010b:20081)

[22] Raphaël Rouquier, Representations of rational Cherednik algebras, Infinite-dimensional aspects of representation theory and applications, Contemp. Math., vol. 392, Amer. Math. Soc., Providence, RI, 2005, pp. 103-131, DOI 10.1090/conm/392/07357. MR2189874 (2007d:20006)

Department of Mathematics, Massachusetts Institute of Technology, Cambridge, Massachusetts 02139

E-mail address: inna.entova@gmail.com 